CERN-TH/2001-305

DESY 01-099

hep-ph/0201036

\title{
The Dipole Formalism for Next-to-Leading Order QCD Calculations with Massive Partons
}

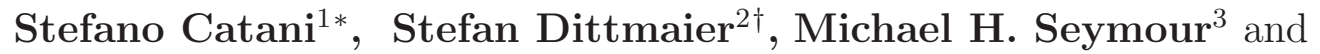 \\ Zoltán Trócsányi ${ }^{4}$ \\ 1 Theory Division, CERN, CH-1211 Geneva 23, Switzerland \\ ${ }^{2}$ Deutsches Elektronen-Synchrotron DESY, D-22603 Hamburg, Germany \\ 3 Theory Group, Department of Physics \& Astronomy, The University of Manchester, \\ Manchester, M13 9PL, U.K. \\ ${ }^{4}$ University of Debrecen and \\ Institute of Nuclear Research of the Hungarian Academy of Sciences \\ H-4001 Debrecen, PO Box 51, Hungary
}

\begin{abstract}
The dipole subtraction method for calculating next-to-leading order corrections in QCD was originally only formulated for massless partons. In this paper we extend its definition to include massive partons, namely quarks, squarks and gluinos. We pay particular attention to the quasi-collinear region, which gives rise to terms that are enhanced by logarithms of the parton masses, $M$. By ensuring that our subtraction cross section matches the exact real cross section in all quasi-collinear regions we achieve uniform convergence both for hard scales $Q \sim M$ and $Q \gg M$. Moreover, taking the masses to zero, we exactly reproduce the previously-calculated massless results. We give all the analytical formulae necessary to construct a numerical program to evaluate the next-to-leading order QCD corrections to arbitrary observables in an arbitrary process.
\end{abstract}

December 2001

${ }^{*}$ On leave of absence from INFN, Sezione di Firenze, Florence, Italy.

${ }^{\dagger}$ Heisenberg fellow of the Deutsche Forschungsgemeinschaft DFG.

${ }^{\ddagger}$ Széchenyi fellow of the Hungarian Ministry of Education. 


\section{Introduction}

Hard-scattering production of heavy quarks and strongly-interacting heavy particles (such as squarks, gluinos and so forth) is of topical interest for Standard Model (SM) and beyond Standard Model physics at high-energy colliders (see, e.g. Refs. [1, 2] and references therein). In order to have reliable theoretical predictions, it is important to control and explicitly compute QCD radiative corrections to these production processes beyond the leading order (LO) approximation in perturbation theory. These computations are very involved, as has been known since the first next-to-leading order (NLO) calculations of heavy-quark production in hadron collisions [3], 4].

In the case of hard-scattering processes that involve only massless QCD partons, the practical feasibility of higher-order computations has been highly simplified by the development of general algorithms [5, 6, 7, 8, 9, 10, 11, 12] to perform NLO calculations. These algorithms, based either on the phase-space slicing method [13] or on the subtraction method [14], start from the process-dependent QCD matrix elements and apply process-independent procedures to isolate and cancel the infrared (soft and collinear) divergences that appear at intermediate steps of the calculation. The final output is a process-independent recipe to construct a modified version of the original matrix elements. The modified matrix elements can directly be integrated (usually by numerical Monte Carlo techniques) over the relevant and process-dependent phase space to compute any infrared and collinear safe observable at NLO.

The NLO algorithm based on the dipole formalism [10] was fully worked out in Ref. [11]. In recent years, it has been implemented in general purpose Monte Carlo codes for the calculation of 3-jet [10] and 4-jet observables [15, 16] in $e^{+} e^{-}$annihilation, 2-jet [11, 17] and 3 -jet [17] final states in deep inelastic lepton-hadron scattering, production of three jets [18], vector-boson pairs [19], vector boson plus massless $b \bar{b}$ [20 and colourless supersymmetric particles 21] at hadron colliders. It has also been applied to the computation of QCD corrections to specific processes, such as 4-fermion final states [22] and forward-backward asymmetries [23] at high-energy $e^{+} e^{-}$colliders and to radiative quarkonium decays [24].

Recent activity has been devoted to extending the dipole formalism by including the effect of massive partons. A first step was performed in Ref. [25] (see also Ref. [26] for the particular case of small fermion masses) by developing the dipole formalism to compute NLO QED radiative corrections to electroweak processes and thus by considering photon radiation from massive charged fermions in arbitrary helicity configurations. The QED version of the dipole formalism was applied to study single-photon radiation in the scattering processes $\gamma \gamma \rightarrow f \bar{f}, e^{-} \gamma \rightarrow e^{-} \gamma$ and $\mu^{+} \mu^{-} \rightarrow \nu_{e} \bar{\nu}_{e}$ [25], to evaluate NLO electroweak corrections to $e^{+} e^{-} \rightarrow W W \rightarrow 4 f$ [27] and to $W$-boson production at hadron colliders 28] and to compute NLO QCD corrections to $e^{+} e^{-} \rightarrow H t \bar{t}$ (and $H b \bar{b}$ ) in the SM and its minimal supersymmetric extension [29]. A QCD extension of the results of Ref. [11] to pro-

cesses involving heavy fermions has been presented in Ref. 30]. In this paper we present our version of the extension of the massless dipole formalism to QCD heavy partons. A small part of the results were anticipated in Ref. [31]. Full results of our formalism have already been applied to the computation of the NLO QCD corrections to the associated production of the SM Higgs boson and a $t \bar{t}$ pair in hadron collisions [32. 
In extending the NLO dipole formalism [11] to arbitrary processes with massive partons, we devote particular attention to its behaviour in the massless limit. As long as we are interested in isolating and cancelling the infrared divergences in the cross section, the extension from massless partons to heavy partons mainly involve kinematical complications due to the finite value of the parton masses. QCD radiation from heavy partons, however, can lead to contributions that, though infrared finite, are proportional to powers of $\ln Q^{2} / M^{2}$, where $M$ is the parton mass and $Q$ is the typical scale of the hard-scattering process. In kinematical configurations where $Q \gg M$, these logarithmically-enhanced contributions (as well as other types of constant contributions discussed in Sect. 2.1) become large and can spoil the numerical convergence of the calculation. In our formulation of the dipole method, we are not mainly concerned with the evaluation of these terms at NLO (or with their resummation to all orders), but rather in minimizing the instabilities that these terms can produce. For instance, in the case of cross sections that are infrared and collinear safe in the massless limit, these logarithmic terms cancel in the final NLO result, but they can still appear at intermediate steps of the calculation (e.g. in the separate evaluation of the real and virtual contributions) thus leading to reduced convergence in numerical implementations. These contributions can be singled out in a process-independent manner, since they are related to the singular behaviour of the QCD matrix elements in the limit $M \rightarrow 0$. This singular behaviour is controlled by quasi-collinear factorization formulae, in the same way that the infrared divergences are controlled by soft and collinear factorization formulae (see Sect. (4). We exploit these factorization properties not only to cancel the infrared divergences, but also to stabilize the NLO algorithm with respect to large numerical contributions that can arise when the hard-scattering kinematics vary from the regions where $Q \sim M$ to those where $Q \gg M$. In the case of processes with no initial-state hadrons (see Sect. 2.1), we set up the method such that, for those cross sections that are infrared and collinear safe in the limit of vanishing parton masses, the NLO algorithm is smooth in the massless limit and, more importantly, numerically stable for any values of the hard-scattering scale. In the case of hadron collision processes (see Sect. 2.2), similar features are achieved provided the NLO partonic calculation is properly matched with the definition of the parton distributions of the colliding hadrons.

In summary, we present a general extension of the dipole formalism that includes different species of strongly interacting massive fermions (quarks, gluinos) and scalars (squarks) with equal or unequal masses (see Sect. 5 and Appendix Q). We develop the formalism by explicitly carrying out the analytical part of the NLO calculation for arbitrary infrared and collinear safe observables in lepton and hadron collisions (see Sect. 6). The discussion of cross sections that involve fragmentation functions of massless or light (with respect to the hard-scattering scale) partons is only sketched, since it does not involve major technical complications with respect to the results presented here and in Ref. [11]. An important general feature of our treatment regards the identification and control of logarithmicallyenhanced contributions of the type $\ln Q^{2} / M^{2}$, which can produce numerical instabilities in kinematical regimes where the hard-scattering scale $Q$ is much larger than the value $M$ of the mass of one or more heavy partons.

This feature, which was also implemented in the case of photon radiation from massive fermions [25], is the main overall difference with respect to other process-independent treatments [25, 26, 30, 7] of massive partons in NLO calculations. There are, however, other general differences. For instance, in Refs. [25, 26] the soft divergences are regular- 
ized by introducing an infinitesimal photon mass (a widespread practice in calculations of electroweak radiative corrections), while we use dimensional regularization. The authors of Ref. [30] consider only fermions as heavy partons and do not consider the cases with different species of massive fermions of unequal masses. The general treatment of Ref. [7] is based on the phase-space slicing method. The authors of Ref. [7] pointed out the relevance of the quasi-collinear limit to control the large terms $\ln Q^{2} / M^{2}$ in the region where $Q \gg M$. They also discussed how these terms can be incorporated in the definition of parton distributions and fragmentation functions of heavy quarks but postponed a detailed treatment to future work.

In the case of massless partons, the dipole formalism was fully developed and described in Ref. [11]. In the present extension to include massive partons, we closely follow the presentation in Ref. [11]. Therefore, we do not repeat all the steps with the same amount of details as in Ref. [11]. The outline of the paper is as follows. In Sect. 2 we discuss the general features of our implementation of massive partons in the dipole formalism. In Sect. 3 we recall the notation and its extension to the massive case. In Sect. 14 we discuss the singular behaviour of the real-emission matrix elements in the soft, collinear and quasi-collinear limits. In Sect. 5 we give the definition of the dipole factorization formulae that smoothly interpolate these limiting regions for any values of the parton masses. The proof that the definition consistently matches the singular behaviour of the QCD matrix elements is not explicitly given: it proceeds along the same lines as in Ref. [11]. In the case of vanishing parton masses, all the dipole factors reduce to those defined in Ref. [11]. In Sect. 5 we also derive the factorization properties of the dipole phase space and perform the corresponding integration of the dipole factorization formulae. Sect. 6 contains the explicit expressions necessary for calculating QCD cross sections in different classes of processes at NLO accuracy. In Sect. 6.1, we write down the final formulae needed to implement our method, while in Sects. 6.2 6.4 we sketch their derivation for processes with no (Sect. 6.2), one (Sect. 6.3) and two (Sect. 6.4) initial-state hadrons. After reading Sect. 3, a reader who is familiar with the method in the massless case and is interested only in setting up a NLO Monte Carlo program for a specific process can find all the relevant results in Sect. 6.1 and in one of the following subsections (depending on the specific process). Sect. 7 contains our conclusions.

We leave some technical details and discussion of explicit examples to the appendices. In Appendix A, we spell out the explicit expression for the integral of the eikonal term. Appendix $\mathrm{B}$ discusses a subtle point in the definition of the $x$-distributions related to the treatment of initial-state singularities in NLO calculations. In Appendix $\mathrm{Q}$, we give the dipole splitting functions that are relevant for SUSY QCD calculations. Finally, we present the three simplest examples of our method in Appendix D. 


\section{The general method and its features in the massless limit}

In this section we briefly review the method of Refs. [10, 11] and describe the main features of our extension to processes with massive partons.

\subsection{Cross sections without identified or initial-state hadrons and their smooth massless limit}

We first consider processes with no initial-state colliding hadrons and no tagged hadrons in the final state. This is the case, for example, of jet production in $e^{+} e^{-}$collisions. Our aim is to calculate a generic infrared- and collinear-safe cross section $\sigma$ with NLO accuracy. Assuming that at the LO there are $m$ QCD partons in the final state, we schematically write

$$
\sigma=\sigma^{\mathrm{LO}}+\sigma^{\mathrm{NLO}}=\int_{m} \mathrm{~d} \sigma^{\mathrm{B}}+\sigma^{\mathrm{NLO}}
$$

where the LO contribution $\mathrm{d} \sigma^{\mathrm{B}}$ is the fully differential Born cross section and the NLO correction $\sigma^{\mathrm{NLO}}$ comprises the real and virtual (one-loop) contributions $\mathrm{d} \sigma^{\mathrm{R}}$ and $\mathrm{d} \sigma^{\mathrm{V}}$ :

$$
\sigma^{\mathrm{NLO}}=\int_{m+1} \mathrm{~d} \sigma^{\mathrm{R}}+\int_{m} \mathrm{~d} \sigma^{\mathrm{V}}
$$

Here the notation for the integrals indicates that the real contribution involves $m+1$ finalstate partons (one QCD parton more than in LO), while the virtual contribution has the $m$-parton kinematics.

After renormalization of the one-loop matrix element involved in $\mathrm{d} \sigma^{\mathrm{V}}$, all the contributions to $\sigma$ are ultraviolet (UV) finite. The Born contribution $\mathrm{d} \sigma^{\mathrm{B}}$ is integrable over the infrared (IR) region of the phase space, but the real and virtual contributions to $\sigma^{\mathrm{NLO}}$ are separately affected by IR divergences produced by soft and collinear partons. Although the IR divergences cancel in the sum on the right-hand side of Eq. (2.2), the separate pieces have to be regularized before any numerical calculation can be attempted. Analytic continuation of the integrals to $d=4-2 \epsilon$ space-time dimensions is the only known gaugeand Lorentz-invariant regularization procedure, but it prevents a straightforward numerical integration. Using dimensional regularization, the IR divergences are replaced by double and single poles, $1 / \epsilon^{2}$ and $1 / \epsilon$, that have to be extracted and cancelled analytically before performing the limit $\epsilon \rightarrow 0$.

The formalism of Ref. [11] deals with the $\epsilon$ poles by using the subtraction method. The general idea of the subtraction method is to introduce an auxiliary cross section $\mathrm{d} \sigma^{\mathrm{A}}$ that has the same pointwise singular behaviour (in $d$ dimensions!) as $\mathrm{d} \sigma^{\mathrm{R}}$. Moreover, $\mathrm{d} \sigma^{\mathrm{A}}$ has to be chosen simple enough, such that it is analytically integrable in $d$ dimensions over the one-parton subspaces that cause the soft and collinear divergences. Thus, without performing any approximations, $\mathrm{d} \sigma^{\mathrm{A}}$ is subtracted from the real contribution and added back to the virtual contribution. Since $\mathrm{d} \sigma^{\mathrm{A}}$ acts as a local counterterm for $\mathrm{d} \sigma^{\mathrm{R}}$, the

\footnotetext{
${ }^{\S} \mathrm{A}$ concise overview of the dipole formalism can also be found in Ref. [33].
} 
difference $\left[\mathrm{d} \sigma^{\mathrm{R}}-\mathrm{d} \sigma^{\mathrm{A}}\right]$ is integrable over the entire $(m+1)$-particle phase space in any number of dimensions and we can safely take the limit $\epsilon \rightarrow 0$. Moreover, since the analytical expression of the performed integral over the singular subspace, $\int_{1} \mathrm{~d} \sigma^{\mathrm{A}}$, explicitly contains all the $\epsilon$ poles that cancel those of the virtual term $\mathrm{d} \sigma^{\mathrm{V}}$, the $\operatorname{sum}\left[\mathrm{d} \sigma^{\mathrm{V}}+\int_{1} \mathrm{~d} \sigma^{\mathrm{A}}\right]$ is also (numerically) integrable in $d=4$ dimensions over the remaining $m$-parton phase space. The final structure of the NLO calculation is

$$
\sigma^{\mathrm{NLO}}=\int_{m+1}\left[\left(\mathrm{~d} \sigma^{\mathrm{R}}\right)_{\epsilon=0}-\left(\mathrm{d} \sigma^{\mathrm{A}}\right)_{\epsilon=0}\right]+\int_{m}\left[\mathrm{~d} \sigma^{\mathrm{V}}+\int_{1} \mathrm{~d} \sigma^{\mathrm{A}}\right]_{\epsilon=0},
$$

and the two terms on the right-hand side are separately integrable. Usually the integrations are not feasible in analytic form, but they can always be carried out numerically. For instance, the calculation can be implemented in a 'partonic Monte Carlo' program, which generates appropriately weighted partonic events with $m+1$ final-state partons and events with $m$ final-state partons.

The method described so far is equally applicable to QCD calculations that involve massless and massive QCD partons. In principle, the extension from the massless to the massive case can be performed in a straightforward way. It is sufficient to overcome the (non-trivial) technical difficulties related to the generalization of $\mathrm{d} \sigma^{\mathrm{A}}$ to massive partons and, in particular, to the analytic evaluation of the integral $\int_{1} \mathrm{~d} \sigma^{\mathrm{A}}$ over the one-particle phase space with mass constraints.

However, in our extension of the formalism of Ref. [11], we require additional properties. To explain the reasons for these additional requirements, it is important to recall that the finite mass $M$ of the QCD partons plays a different physical role in different physical processes. In some processes (e.g. the total cross section for heavy-quark hadroproduction [3]) the finite (and large) value of $M$ has the essential role of setting the hard scale of the cross section. In these cases the massless limit $M \rightarrow 0$ is IR unstable and the corresponding cross section cannot be computed in QCD perturbation theory. In other processes (e.g. the production of heavy-flavoured jets in $e^{+} e^{-}$annihilation [34]), instead, the hard scale $Q$ is independent of the mass $M$ and the latter has only the role of an auxiliary (though important) kinematical scale. These processes are IR stable in the massless limit, that is, when $M \rightarrow 0$ the cross section is still infrared- and collinear-safe and, thus, perturbatively computable.

The processes that are perturbatively stable in the massless limit are often studied in kinematical regions where the typical hard scale $Q$ is much larger than the mass $M$ of one (or more) of the heavy partons. In this regime the integral of the real term $\mathrm{d} \sigma^{\mathrm{R}}(M)$ of the NLO cross section in Eq. (2.2) leads to contributions of the type

$$
\int_{m+1} \mathrm{~d} \sigma^{\mathrm{R}}(M) \rightarrow \int_{0}^{Q^{2}} \mathrm{~d} \mathbf{q}_{\perp}^{2}\left(\mathbf{q}_{\perp}^{2}\right)^{-\epsilon} \frac{1}{\mathbf{q}_{\perp}^{2}+M^{2}} \underset{Q \gg M}{\widetilde{l n}} \ln \frac{Q^{2}}{M^{2}}+\mathrm{O}(\epsilon)
$$

and

$$
\int_{m+1} \mathrm{~d} \sigma^{\mathrm{R}}(M) \rightarrow \int_{0}^{Q^{2}} \mathrm{~d} \mathbf{q}_{\perp}^{2}\left(\mathbf{q}_{\perp}^{2}\right)^{-\epsilon} \frac{M^{2}}{\left[\mathbf{q}_{\perp}^{2}+M^{2}\right]^{2}} \underset{Q \gg M}{\widetilde{ }} M^{2} \frac{1}{M^{2}}+\mathrm{O}(\epsilon)
$$

where $\mathbf{q}_{\perp}$ generically denotes the typical transverse momentum of the heavy parton with mass $M$. Since these contributions are finite when $\epsilon \rightarrow 0$, naively, they would not require 
any special treatments within the subtraction method. However, this could lead to serious numerical problems.

The problems are evident in the case of the contribution in Eq. (2.4), which is very large when $Q \gg M$. When computing the NLO cross section, this large contribution would appear in the first term (the $(m+1)$-parton integral) on the right-hand side of Eq. (2.3) and it would be compensated by an equally large (but with opposite sign) logarithmic contribution arising from the second term (the $m$-parton integral). Owing to the presence of several large (although compensating) contributions, a similar naive procedure would lead to instabilities in any numerical implementations of the NLO calculation. The numerical instabilities would increase by increasing the ratio $Q / M$ and, in particular, they would prevent from performing the massless limit.

The contribution in Eq. 2.5) may appear harmless, since it approaches a constant (finite) value when $M / Q \rightarrow 0$. However, the constant behaviour is obtained by combining the factor $M^{2}$ from the numerator of the integrand with the factor $1 / M^{2}$ from the integral of the denominator. Owing to the presence of a linearly divergent (in the limit $M^{2} / Q^{2} \rightarrow 0$ ) integral, the contribution in Eq. (2.5) cannot be evaluated numerically by using standard Monte Carlo techniques, since its variance increases linearly with $Q^{2} / M^{2}$. Finite integrals with infinite variance (e.g. integrands with square root singularities) often occur in NLO calculations: they can be treated within Monte Carlo methods by applying importance sampling procedures. In this respect, the term in Eq. (2.5) poses no additional conceptual problems. However, our main point is that the variance of the integral in Eq. (2.5) is not uniform in $M$ : it varies from a finite value when $M=\mathrm{O}(Q)$ to a divergent value when $M / Q \rightarrow 0$. The presence of these type of contributions may thus prevent from straightforwardly constructing a 'partonic Monte Carlo' program that performs equally well in the two kinematical regimes $M=\mathrm{O}(Q)$ and $M / Q \ll 1$.

To avoid these numerical problems in the calculation of cross sections that are IR stable in the massless limit, we set up our massive-parton formalism by choosing the auxiliary cross section $\mathrm{d} \sigma^{\mathrm{A}}(M)$ in such a way that the following property is fulfilled:

$$
\lim _{M \rightarrow 0} \int_{m+1}\left[\left(\mathrm{~d} \sigma^{\mathrm{R}}(M)\right)_{\epsilon=0}-\left(\mathrm{d} \sigma^{\mathrm{A}}(M)\right)_{\epsilon=0}\right]=\int_{m+1}\left[\left(\mathrm{~d} \sigma^{\mathrm{R}}(M=0)\right)_{\epsilon=0}-\left(\mathrm{d} \sigma^{\mathrm{A}}(M=0)\right)_{\epsilon=0}\right] .
$$

Note that, to avoid the problems related to the large logarithmic contributions in Eq. (2.4), it is sufficient to impose that the integral of the subtracted cross section on the left-hand side of Eq. (2.6) is finite when $M \rightarrow 0$. Equation (2.6) is, instead, a stronger constraint. It implies that, in the evaluation of the subtracted cross section, the massless limit (or, more generally, the limit $M / Q \rightarrow 0)$ commutes with the $(m+1)$-parton integral. This guarantees that $\left[\mathrm{d} \sigma^{\mathrm{R}}(M)-\mathrm{d} \sigma^{\mathrm{A}}(M)\right]$ does not contain integrands of the type in Eq. (2.5).

Once Eq. (2.6) is satisfied, the $(m+1)$-parton and $m$-parton contributions to the NLO cross section in Eq. (2.3) separately have smooth behaviour when $M \rightarrow 0$. This behaviour helps to compute the cross section numerically in the kinematical regimes where $Q \gg M$. It can also be used to easily check that, in the massless limit, the massive-parton calculation correctly agrees with the corresponding result obtained in the exactly massless case.

The IR divergent contributions to the real cross section $\mathrm{d} \sigma^{\mathrm{R}}$ are process independent. By this we mean that, in the soft and collinear limits, $\mathrm{d} \sigma^{\mathrm{R}}$ is given by the corresponding 
(process-dependent) Born-level cross section $\mathrm{d} \sigma^{\mathrm{B}}$ times universal (process-independent) singular factors. Owing to these soft and collinear factorization properties [35, 36], it is possible to give general prescriptions for constructing the auxiliary cross section $\mathrm{d} \sigma^{\mathrm{A}}$ in a process-independent manner.

Within the dipole formalism [11], $\mathrm{d} \sigma^{\mathrm{A}}$ is constructed by a sum over different contributions, named dipoles. Each dipole contribution describes soft and collinear radiation from a pair of ordered partons. The first parton is called emitter and the second spectator, since only the kinematics of the former leads to the IR singularities. The dipole configurations can be thought of as being obtained by an effective two-step process: using the Born-level cross section, an $m$-parton configuration is first produced and the emitter and spectator are singled out in all possible ways; then the emitter decays into two partons and the spectator, which contains information on the colour and spin correlations of the real cross section $\mathrm{d} \sigma^{\mathrm{R}}$, is used to balance momentum conservation. The auxiliary cross section $\mathrm{d} \sigma^{\mathrm{A}}$ can symbolically be written as

$$
\mathrm{d} \sigma^{\mathrm{A}}=\sum_{\text {dipoles }} \mathrm{d} \sigma^{\mathrm{B}} \otimes \mathrm{d} V_{\text {dipole }}
$$

where the dipole factors $\mathrm{d} V_{\text {dipole }}$ describe the two-parton decays of the emitters. These factors are universal and can be obtained from the QCD factorization formulae (including the associated colour and spin correlations, as denoted by the symbol $\otimes$ ) in the soft and collinear limits.

The product structure in Eq. (2.7) is made possible by the factorization of QCD amplitudes on soft and collinear poles and by a suitable factorized definition of the phase space. It permits a factorizable mapping from the $(m+1)$-parton phase space to an $m$-parton subspace, identified by the partonic variables in $\mathrm{d} \sigma^{\mathrm{B}}$, times a single-parton phase space, identified by the partonic variables in $\mathrm{d} V_{\text {dipole }}$. The single-parton phase space is processindependent: it describes the two-parton decay of the dipole and embodies the kinematical dependence on the degrees of freedom that lead to the IR singularities. This mapping makes $\mathrm{d} V_{\text {dipole }}$ fully integrable analytically and in a process-independent manner. We can symbolically write:

$$
\int_{m+1} \mathrm{~d} \sigma^{\mathrm{A}}=\sum_{\text {dipoles }} \int_{m} \mathrm{~d} \sigma^{\mathrm{B}} \otimes \int_{1} \mathrm{~d} V_{\text {dipole }}=\int_{m}\left[\mathrm{~d} \sigma^{\mathrm{B}} \otimes \boldsymbol{I}\right],
$$

where the universal factor $\boldsymbol{I}$ is defined by

$$
\boldsymbol{I}=\sum_{\text {dipoles }} \int_{1} \mathrm{~d} V_{\text {dipole }}
$$

and contains all the $\epsilon$ poles that are necessary to cancel the (equal and with opposite sign) poles in $\mathrm{d} \sigma^{\mathrm{V}}$. As a byproduct of this cancellation mechanism, Eq. (2.8) can also be used to indirectly derive explicit information on the $\epsilon$ singularities in the virtual contribution. Since $\mathrm{d} \sigma^{\mathrm{V}}$ and $\mathrm{d} \sigma^{\mathrm{B}}$ are respectively obtained from one-loop and tree-level QCD amplitudes, the factorization structure on the right-hand side of Eq. (2.8) implies that the IR divergences of the one-loop amplitudes can be obtained from the corresponding tree amplitudes in terms of a universal factorization formula [5, 37, 11]. 
The structure of the final NLO result is given as follows in terms of two contributions, $\sigma^{\mathrm{NLO}\{m+1\}}$ and $\sigma^{\mathrm{NLO}\{m\}}$, with $(m+1)$-parton and $m$-parton kinematics, respectively, which are separately finite and integrable in four space-time dimensions:

$$
\begin{aligned}
\sigma^{\mathrm{NLO}} & =\sigma^{\mathrm{NLO}\{m+1\}}+\sigma^{\mathrm{NLO}\{m\}} \\
& =\int_{m+1}\left[\left(\mathrm{~d} \sigma^{\mathrm{R}}\right)_{\epsilon=0}-\left(\sum_{\text {dipoles }} \mathrm{d} \sigma^{\mathrm{B}} \otimes \mathrm{d} V_{\text {dipole }}\right)_{\epsilon=0}\right]+\int_{m}\left[\mathrm{~d} \sigma^{\mathrm{V}}+\mathrm{d} \sigma^{\mathrm{B}} \otimes \boldsymbol{I}\right]_{\epsilon=0} .
\end{aligned}
$$

Equation (2.10) represents the dipole formalism implementation of the general subtraction formula (2.3).

The explicit expressions of the dipole factors $\mathrm{d} V_{\text {dipole }}$ and $\boldsymbol{I}$ for all the cross sections with massless QCD partons were obtained in Ref. [11]. In this paper we present their generalization to the case of massive QCD partons in the final state. As in Ref. [11], the dipole factors $\mathrm{d} V_{\text {dipole }}$ are constructed by starting from the QCD factorization formulae in the soft and collinear limits. Moreover, to implement the smoothness condition in Eq. (2.6), the dipole factors have to match the behaviour of the QCD matrix elements in the so-called quasi-collinear limit [31]. The collinear limit describes the splitting process of one massless parton in two massless partons when the relative transverse momentum $\mathbf{q}_{\perp}$ of the latter vanishes. The quasi-collinear limit (see Sect. 4.2) is a generalization of the collinear limit to the splitting processes of massive partons. It is obtained by letting $\mathbf{q}_{\perp}$ and the parton masses vanish uniformly, i.e. we consider the limit $\mathbf{q}_{\perp}, M \rightarrow 0$ at fixed ratio $\mathbf{q}_{\perp} / M$. In the quasi-collinear limit, the integrands of the type in Eqs. (2.4) and (2.5) are formally regarded as being as singular as those that are proportional to $1 / \mathbf{q}_{\perp}^{2}$ and lead to the collinear divergences. Therefore, constructing the dipole factors in Eq. (2.7) in such a way that they have the same quasi-collinear limit as the real cross section $\mathrm{d} \sigma^{\mathrm{R}}$, our (subtracted) $(m+1)$-parton contribution, $\sigma^{\mathrm{NLO}\{m+1\}}$, to Eq. (2.10) fulfils the smoothness condition in Eq. (2.6). In addition, the dipole factors of the present paper exactly coincide with those of Ref. [11] in the massless limit. We do that for the practical purpose of facilitating a direct comparison with the results in Ref. [11].

Analogously to the massless-parton case, the cancellation of the $\epsilon$ poles in the $m$-parton contribution $\sigma^{\mathrm{NLO}\{m\}}$ to Eq. (2.10) can be exploited to derive a universal factorization formula that controls the IR divergences of any one-loop amplitude with massless and massive partons. Having fulfilled the smoothness condition (2.6) and performing the analytical integration over the one-parton phase space in Eq. (2.9) uniformly in the parton masses, our $\sigma^{\mathrm{NLO}\{m\}}$ can be used to obtain such a factorization formula in a form that explicitly exhibits not only the $\epsilon$ poles, but also (i) the logarithmic terms (of the type $\ln ^{2} M$ and $\ln M$ ) that become singular when $M \rightarrow 0$ and (ii) the constant (finite) terms that originate in the calculation of the one-loop amplitude from the non-commutativity of the limits $M \rightarrow 0$ and $\epsilon \rightarrow 0$. This result was anticipated in Ref. [31].

\subsection{Cross sections in hadron collisions}

The general procedure we have outlined so far applies to all processes with no initialstate hadrons (for instance, jets and heavy-quark production in $e^{+} e^{-}$annihilation). In 
lepton-hadron and hadron-hadron collision processes, the hadronic cross section is computed by convoluting the corresponding partonic cross section $\sigma\left(x p_{\text {had }}\right)$ with the (processindependent) parton distributions $f(x)$ of the incoming hadrons. Here, $p_{\text {had }}$ and $x$ generically denote the momentum of the colliding hadron and the momentum fraction carried by the colliding parton, respectively. The presence of initial-state partons, carrying a well defined momentum, makes the partonic subprocess collinear unsafe, so the partonic cross section cannot naively be computed in QCD perturbation theory. However, provided the incoming parton is massless, the universal factorization theorem [38 of collinear singularities holds, and perturbative QCD can still be applied. The initial-state collinear divergences of the partonic cross section can be factorized and reabsorbed in the definition of the nonperturbative parton distributions. This procedure can consistently be carried out at any perturbative order. In particular, at NLO the perturbatively-computable partonic cross section is given by

$$
\sigma^{\mathrm{NLO}}(p)=\int_{m+1} \mathrm{~d} \sigma^{\mathrm{R}}(p)+\int_{m} \mathrm{~d} \sigma^{\mathrm{V}}(p)+\int_{m} \mathrm{~d} \sigma^{\mathrm{C}}(p)
$$

where

$$
\int_{m} \mathrm{~d} \sigma^{\mathrm{C}}(p)=\int_{0}^{1} \mathrm{~d} x \int_{m} \mathrm{~d} \sigma^{\mathrm{B}}(x p) \Gamma(x) .
$$

Comparing Eq. (2.2) with Eq. (2.11), we see that the latter contains the additional collinear counterterm $\mathrm{d} \sigma^{\mathrm{C}}(p)$, which arises from the redefinition of the parton distributions. As symbolically written in Eq. (2.12), the collinear counterterm is given by the convolution of the Born cross section with a process-independent factor $\Gamma(x)$, which is divergent when $\epsilon \rightarrow 0$.

We have emphasized the fact that the partons that initiate the hard-scattering subprocess have to be massless. The reason is that in the presence of two or more incoming massive partons the Bloch-Nordsieck mechanism [39] of cancellation of soft divergences is violated in QCD [40]. The violation is due to non-cancelled soft divergences that are proportional to the mass $M$ of the incoming partons and are process dependent. Although the violation occurs starting from next-to-next-to-leading order (NNLO), its process dependence spoils the general validity of the factorization theorem [38]. Since the factorization theorem cannot formally be used to perturbatively define and compute partonic cross sections with incoming massive partons, we do not consider these cross sections.

Note, however, that our distinction between massless and massive incoming partons has only a formal meaning. By 'mass' of the incoming parton we do not mean the actual physical mass of the parton. We only mean that the perturbative calculation is performed by setting this mass equal to zero. The practical calculational procedure is as follows. If the physical mass of a parton is much smaller than the typical value $Q$ of the hard-scattering scale in the process, this parton has to be treated as a massless parton and included both in the initial and in the final state. If the case is not so, the parton has to be treated as massive and not considered as an incoming parton. We discuss this point further at the end of this subsection.

The subtraction method and the dipole formalism can be used to compute the NLO partonic cross section in Eq. (2.11). Using the dipole formalism, the auxiliary cross section

\footnotetext{
"By general we mean to all perturbative orders and for arbitrary non-vanishing values of the mass of the incoming partons.
} 
$\mathrm{d} \sigma^{\mathrm{A}}$ still has the form in Eq. (2.7) and the sum over the dipoles is extended [11] to include initial-state dipoles that describe the collinear decays of any initial-state massless parton (the emitter) into two massless partons. Furthermore, in considering the final-state emitters, the sum over spectators has to be extended to include initial-state massless partons, leading to dipole kinematics that differ from those with a final-state spectator. In the case with only massless partons in the final state, the initial-state dipoles were worked out in Ref. [11]. In this paper we extend the results of Ref. [11] to include the case with massive partons in the final state.

Independently of the fact that the final-state partons are massless or massive, the initialstate dipoles can be integrated over the collinear region. Their integral cancels the divergences of the collinear counterterm $\mathrm{d} \sigma^{\mathrm{C}}(x p)$, and the final result for NLO cross section in Eq. (2.11) can be written symbolically as

$$
\begin{aligned}
\sigma^{\mathrm{NLO}}(p) & =\sigma^{\mathrm{NLO}\{m+1\}}(p)+\sigma^{\mathrm{NLO}\{m\}}(p)+\int_{0}^{1} d x \hat{\sigma}^{\mathrm{NLO}\{m\}}(x ; x p) \\
& =\int_{m+1}\left[\left(\mathrm{~d} \sigma^{\mathrm{R}}(p)\right)_{\epsilon=0}-\left(\sum_{\text {dipoles }} \mathrm{d} \sigma^{\mathrm{B}}(p) \otimes \mathrm{d} V_{\text {dipole }}\right)_{\epsilon=0}\right] \\
& +\int_{m}\left[\mathrm{~d} \sigma^{\mathrm{V}}(p)+\mathrm{d} \sigma^{\mathrm{B}}(p) \otimes \boldsymbol{I}\right]_{\epsilon=0}+\int_{0}^{1} d x \int_{m}\left[\mathrm{~d} \sigma^{\mathrm{B}}(x p) \otimes(\boldsymbol{P}+\boldsymbol{K})(x)\right]_{\epsilon=0} .
\end{aligned}
$$

The contributions $\sigma^{\mathrm{NLO}\{m+1\}}(p)$ and $\sigma^{\mathrm{NLO}\{m\}}(p)$ (with $(m+1)$-parton and $m$-parton kinematics, respectively) are analogous to those in Eq. (2.10). The last term on the right-hand side of Eq. (2.13) is a finite (in four dimensions) remainder that is left after cancellation of the $\epsilon$ poles of the collinear counterterm in Eq. (2.12). This term involves a cross section $\hat{\sigma}^{\mathrm{NLO}\{m\}}(x ; x p)$ with $m$-parton kinematics and an additional one-dimensional integration with respect to the longitudinal momentum fraction $x$. This integration arises from the convolution of the Born-level cross section $\mathrm{d} \sigma^{\mathrm{B}}(x p)$ with $x$-dependent functions $\boldsymbol{P}$ and $\boldsymbol{K}$ that are universal and finite for $\epsilon \rightarrow 0$. The explicit expressions of $\boldsymbol{P}$ and $\boldsymbol{K}$ in the massless case were given in Ref. [1]. In this paper we obtain their generalization to the case of partonic cross sections with massive partons in the final state.

In Ref. [11] an extensive discussion was devoted to a fully detailed treatment of cross sections with identified hadrons in the final state, i.e. cross sections that involve the introduction of parton fragmentation functions of the outgoing hadrons. For simplicity, in this paper we do not present an analogous discussion of these cross sections, since the extension to include the associated production of final-state heavy partons is straightforward. More precisely, we can consider two main kinematical configurations according to whether the momentum $Q$ of the final-state massive parton is a) not observed or comparable to its mass $M$ and $b$ ) much larger than its mass. In the case $a$ ), the heavy parton does not require additional (with respect to other cross sections) special treatment, apart from it being included as spectator in the final-state dipoles that are introduced [11] to describe the collinear decay of the massless emitter that undergoes final-state fragmentation. In the case $b$ ), a proper treatment of the large contributions $\ln Q^{2} / M^{2}$ is required, which can be simply achieved by considering the massive parton as effectively massless and by introducing its (process-independent) perturbative fragmentation function 41].

Considering the case of cross section calculations in processes with no initial-state hadrons, in Sect. 2.1 we have discussed how we set up the dipole formalism with mas- 
sive partons to guarantee a smooth and numerically stable interpolation throughout the kinematical region where the hard-scattering scale $Q$ varies from $Q \sim M$ to $Q \gg M$. In hadron collisions, a QCD parton with a non-vanishing physical mass $M$ has to be regarded as effectively massive or massless depending on whether $Q \sim M$ or $Q \gg M$. Moreover, the transition between the former to the latter region needs the introduction and schemedependent definition of the parton distribution of the effectively massless parton. Evidently, a smooth interpolation between the two regions requires a proper and careful matching between the definition of the partonic cross section and that of the parton distribution. This general theoretical issue has been the subject of many investigations in the recent literature (see e.g. the list of references in Ref. [42]), and several practical implementations are available, mostly in the context of deep-inelastic heavy-quark production. Once the matching conditions and the parton distributions within a certain scheme are precisely specified, smoothness conditions similar to Eq. (2.6) can be imposed also on NLO calculations of cross sections that involve parton distributions and fragmentation functions. The procedure outlined in Sect. 2.1 to guarantee the validity of Eq. (2.6) can thus be applied to set up the dipole formalism such that it performs in a manner that is smooth and numerically stable with respect to large variations of the ratio $Q / M$. This is left to future studies.

\subsection{Summary of the general method}

The starting points of the calculation of QCD radiative corrections at NLO are the treelevel and one-loop matrix elements that respectively enter in the expression of the cross section contributions $\mathrm{d} \sigma^{\mathrm{R}}$ and $\mathrm{d} \sigma^{\mathrm{V}}$ in Eq. (2.2) (or Eq. (2.11)). The separate integration of these matrix elements is not trivial because of their infrared divergences. The goal of the dipole formalism is to construct 'effective matrix elements' that can straightforwardly be integrated in four space-time dimensions. The final output of the formalism is summarized in Eq. (2.10) (or Eq. (2.13)), which give the expression of the NLO cross sections in terms of the 'effective matrix elements'. The formalism provides explicit expressions for the universal factors $\mathrm{d} V_{\text {dipole }}$ and $\boldsymbol{I}$ (and $\boldsymbol{P}, \boldsymbol{K}$ ), which we present in Sect. 6. Having these factors at our disposal, the only other ingredients necessary for the full NLO calculation are simply related to the evaluation of the original matrix elements. We need:

- a set of independent colour projections of the matrix element squared at the Born level, summed over parton polarizations, in $d$ dimensions;

- the one-loop contribution $\mathrm{d} \sigma^{\mathrm{V}}$ in $d$ dimensions;

- an additional projection of the Born-level matrix element over the helicity of each external gluon in four dimensions;

- the real emission contribution $\mathrm{d} \sigma^{\mathrm{R}}$ in four dimensions.

Since the comment presented in the 'Note added' section of Ref. [11] is valid independently of the value of the parton masses, this list can be simplified if one uses the dimensionalreduction scheme for regularizing the one-loop matrix elements. In this case the Born-level calculation in the first item above can directly be carried out in four space-time dimensions. 


\section{Notation}

\subsection{Matrix elements}

We consider processes with coloured particles (partons) in the initial and final states. Any number of additional non-coloured particles is allowed, too, but they will be suppressed in the notation. Partons in the initial state are labelled by $a, b, \ldots$, partons in the final state by $i, j, k, \ldots$; generically we write $\{i ; a\}$ for all partons. When we do not make a distinction between initial- and final-state partons, we use the common labels $I, J, \ldots$ and we shortly write $\{I\}=\{i ; a\}$.

The colour indices of the partons are denoted by $c_{a}, c_{i}$, which range over $1, \ldots, N_{c}^{2}-1$ for gluons (or any other partons, such as gluinos, in the adjoint representation of the gauge group) and over $1, \ldots, N_{c}$ for quarks and antiquarks (or any other partons, such as squarks, in the fundamental representation). Spin indices are generically denoted by $s_{a}, s_{i}$. As in Ref. [1]], we formally introduce an orthogonal basis of unit vectors $\left|\left\{c_{i} ; c_{a}\right\}\right\rangle \otimes\left|\left\{s_{i} ; s_{a}\right\}\right\rangle$ in the space of colour and spin, in such a way that an amplitude $\mathcal{M}^{\left\{c_{i}, s_{i} ; c_{a}, s_{a}\right\}}\left(\left\{p_{i} ; p_{a}\right\}\right)$ with definite colour, spin and momenta $\left\{p_{i}, p_{a}\right\}$ can be written as

$$
\mathcal{M}^{\left\{c_{i}, s_{i} ; c_{a}, s_{a}\right\}}\left(\left\{p_{i} ; p_{a}\right\}\right) \equiv\left(\prod_{b} \sqrt{n_{c}(b)}\right)\left(\left\langle\left\{c_{i} ; c_{a}\right\}\left|\otimes\left\langle\left\{s_{i} ; s_{a}\right\}\right|\right) \mid\{i ; a\}\right\rangle .\right.
$$

Thus $|\{i ; a\}\rangle$ is an abstract vector in colour and spin space, and its normalization is fixed by

including a factor of $1 / \sqrt{n_{c}(b)}$ for each initial-state parton $b$ carrying $n_{c}(b)$ colour degrees of freedom. Then the squared amplitude summed over colours and spins and averaged over initial-state colours is

$$
\frac{1}{\prod_{b} n_{c}(b)}\left|\mathcal{M}\left(\left\{p_{i} ; p_{a}\right\}\right)\right|^{2}=\langle\{i ; a\} \mid\{i ; a\}\rangle
$$

Colour interactions at the QCD vertices are represented by associating colour charges $\boldsymbol{T}_{i}$ or $\boldsymbol{T}_{a}$ with the emission of a gluon from each parton $i$ or $a$. The colour charge $\boldsymbol{T}_{i}=\left\{T_{i}^{n}\right\}$ is a vector with respect to the colour indices $n$ of the emitted gluon and an $S U\left(N_{c}\right)$ matrix with respect to the colour indices of the parton $i$; analogously $\boldsymbol{T}_{a}$ describes gluon emission from the initial-state parton $a$. More precisely, for a final-state parton $i$ the action onto the colour space is defined by

$$
\left\langle c_{1}, \ldots, c_{i}, \ldots, c_{m}\left|T_{i}^{n}\right| b_{1}, \ldots, b_{i}, \ldots, b_{m}\right\rangle=\delta_{c_{1} b_{1}} \ldots T_{c_{i} b_{i}}^{n} \ldots \delta_{c_{m} b_{m}}
$$

where $T_{c b}^{n}$ is the colour-charge matrix in the representation of the final-state particle $i$, i.e. $T_{c b}^{n}=\mathrm{i} f_{c n b}$ if $i$ is a gluon or a gluino, $T_{\alpha \beta}^{n}=t_{\alpha \beta}^{n}$ if $i$ is a (s)quark and $T_{\alpha \beta}^{n}=-t_{\beta \alpha}^{n}$ if $i$ is an anti(s)quark. The colour-charge operator of an initial-state parton $a$, for which a relation analogous to Eq. (3.3) holds, is defined by crossing symmetry, that is by $T_{\alpha \beta}^{n}=-t_{\beta \alpha}^{n}$ if $a$ is a (s)quark and $T_{\alpha \beta}^{n}=t_{\alpha \beta}^{n}$ if $a$ is an anti(s)quark. Using this notation, we also define the square of colour-correlated tree-amplitudes with $m$ final-state partons, $\left|\mathcal{M}_{m}^{i, k}\right|^{2}$, as follows

$$
\begin{aligned}
\left|\mathcal{M}_{m}^{j, k}\right|^{2} & \equiv\left(\prod_{b} n_{c}(b)\right){ }_{m}\left\langle\{i, a\}\left|\boldsymbol{T}_{j} \cdot \boldsymbol{T}_{k}\right|\{i, a\}\right\rangle_{m} \\
& =\left[\mathcal{M}_{m}^{a_{1} \ldots b_{j} \ldots b_{k} \ldots a_{m}}\left(\left\{p_{i} ; p_{a}\right\}\right)\right]^{*} T_{b_{j} a_{j}}^{n} T_{b_{k} a_{k}}^{n} \mathcal{M}_{m}^{a_{1} \ldots a_{j} \ldots a_{k} \ldots a_{m}}\left(\left\{p_{i} ; p_{a}\right\}\right),
\end{aligned}
$$


and analogously for the cases in which $j$ and/or $k$ are replaced by initial-state partons.

In our notation, each vector $|\{i ; a\}\rangle$ is a colour-singlet state, so colour conservation is simply

$$
\left(\sum_{j} \boldsymbol{T}_{j}+\sum_{b} \boldsymbol{T}_{b}\right)|\{i ; a\}\rangle=0
$$

where the sum over $j$ and $b$ extends over all the external partons of the state vector $|\{i ; a\}\rangle$ in the final and initial state, respectively.

The colour-charge algebra for the product $\left(\boldsymbol{T}_{i}\right)^{n}\left(\boldsymbol{T}_{j}\right)^{n} \equiv \boldsymbol{T}_{i} \cdot \boldsymbol{T}_{j}$ is:

$$
\boldsymbol{T}_{i} \cdot \boldsymbol{T}_{j}=\boldsymbol{T}_{j} \cdot \boldsymbol{T}_{i} \quad \text { if } i \neq j ; \quad \boldsymbol{T}_{i}^{2}=C_{i},
$$

and analogously for initial-state partons. Here $C_{i}$ is the quadratic Casimir operator in the representation of particle $i$ and we have $C_{\mathrm{F}}=T_{\mathrm{R}}\left(N_{\mathrm{c}}^{2}-1\right) / N_{\mathrm{c}}=\left(N_{\mathrm{c}}^{2}-1\right) /\left(2 N_{\mathrm{c}}\right)$ in the fundamental and $C_{\mathrm{A}}=2 T_{\mathrm{R}} N_{\mathrm{c}}=N_{\mathrm{c}}$ in the adjoint representation, i.e. we are using the customary normalization $T_{\mathrm{R}}=1 / 2$.

\subsection{Dimensional regularization, one-loop amplitudes and renor- malization}

We employ dimensional regularization in $d=4-2 \epsilon$ space-time dimensions to regulate both the IR and UV divergences. More precisely, we use conventional dimensional regularization (CDR), where quarks (spin- $\frac{1}{2}$ Dirac fermions) possess 2 spin polarizations, gluons have $d-2$ helicity states and all particle momenta are taken as $d$-dimensional. The $d$-dimensional phase space for $m$ outgoing particles with momenta $p_{1}, \ldots, p_{m}$, masses $m_{1}, \ldots, m_{m}$ and total momentum $P$ is denoted by

$$
\mathrm{d} \phi_{m}\left(p_{1}, \ldots, p_{m} ; P\right)=\left[\prod_{i=1}^{m} \frac{\mathrm{d}^{d} p_{i}}{(2 \pi)^{d-1}} \delta_{+}\left(p_{i}^{2}-m_{i}^{2}\right)\right](2 \pi)^{d} \delta^{(d)}\left(p_{1}+\ldots+p_{m}-P\right) .
$$

The dimensional-regularization scale, which appears in the calculation of the matrix elements, is denoted by $\mu$. The dependence on $\mu$ cancels after having combined the matrix elements in the NLO calculation of physical cross sections, although the latter eventually depend on the renormalization and factorization scales $\mu_{R}$ and $\mu_{F}$. To avoid a cumbersome notation, we therefore set $\mu_{R}=\mu$, while $\mu$ and $\mu_{F}$ will differ in general.

The virtual contribution $\mathrm{d} \sigma^{\mathrm{V}}$ to the NLO cross section is proportional to the real part of the interference between the tree-level and one-loop matrix elements. Although this interference is not positive definite, we denote it by $\left|\mathcal{M}\left(\left\{p_{i}, p_{a}\right\}\right)\right|_{(1-\text { loop })}^{2}$, since it represents the one-loop correction to the square of the tree-level matrix element. Actually, $\mathrm{d} \sigma^{\mathrm{V}}$ is proportional to the renormalized one-loop correction $\left|\mathcal{M}\left(\left\{p_{i}, p_{a}\right\}\right)\right|_{(1-\mathrm{loop})}^{2}$, which is obtained from the corresponding bare quantity by adding ultraviolet counterterms that implement charge (coupling) and mass renormalization. The renormalized QCD coupling at the renor-

malization scale $\mu$ is shortly denoted by $\alpha_{\mathrm{s}}$. It can be defined either in the customary $\overline{\mathrm{MS}}$ renormalization scheme or in the renormalization scheme of Ref. 43, which differs from 
the $\overline{\mathrm{MS}}$ scheme in the treatment of heavy partons and guarantees their decoupling in the infinite mass limit. The renormalized mass parameters (i.e. parton masses and related parameters such as those which appear in Yukawa couplings) are denoted by $m_{i}$ and can refer either to the $\overline{\mathrm{MS}}$ renormalization scheme or to the pole-mass definition. The results of this paper are independent of these different renormalization prescriptions. In particular, since these prescriptions coincide in the limit of vanishing parton masses, they do not affect the massless limit of IR stable cross sections.

Once renormalization has been performed, the one-loop contribution $|\mathcal{M}|_{(1-\text { loop })}^{2}$ no longer contains $1 / \epsilon$ poles of UV origin, but it still contains $1 / \epsilon$ poles and finite terms of IR origin. The latter depend on the dimensional regularization procedure used for evaluating the loop integral. The regularization-scheme dependence of the virtual corrections has to be consistently matched to that of the real ones, to guarantee the regularization-scheme independence of the NLO cross section. Since in this paper we deal with the real corrections by using the CDR scheme, we need the result for $|\mathcal{M}|_{(1-\text { loop })}^{2}$ within this regularization scheme. One-loop matrix elements are sometimes evaluated by using dimensional-regularization prescriptions that differ from CDR (the difference can be due to the dimensionality of the momenta of the external particles and/or to the number of polarizations of both external and internal particles). If $|\mathcal{M}|_{(1-\text { loop })}^{2}$ is known in a scheme that is different from CDR, its expression in the CDR scheme can be obtained by introducing a correction proportional to the corresponding tree-level amplitude. The correction term for several different regularization schemes can be found in Refs. [44, 45] for the massless-parton case and in Ref. [31] for the general case with massless and massive partons.

\section{Factorization in the soft and (quasi-)collinear limits}

We consider a generic tree-level matrix element $\mathcal{M}_{m+1}$ with $m+1$ massive or massless QCD partons in the final state and up to two massless initial-state partons. At NLO, the dependence of the squared matrix element $\left|\mathcal{M}_{m+1}\right|^{2}$ on the momenta of the final-state partons is singular in two different situations. The first situation occurs in the soft region, where the momentum of a final-state gluon tends to zero in any fixed direction. The second situation corresponds to the (quasi-)collinear region, where a final-state parton becomes collinear to another (initial- or final-state) parton.

\subsection{Soft limit}

In the soft region the momentum $p_{j}$ of a final-state gluon $j$ tends to zero in any fixed direction $q$, i.e. $p_{j}=\lambda q$ with $\lambda \rightarrow 0$ for fixed $q$. In this limit, the squared matrix element diverges as $1 / \lambda^{2}$ and its divergent part can be computed in terms of the eikonal current of the soft gluon [35]. As described in Ref. [11] in more detail, using partial fractioning and colour conservation, the singular behaviour of $\left|\mathcal{M}_{m+1}\right|^{2}$ can be written as a sum over 
emitter-spectator $(I-K)$ pairs,

$$
\begin{aligned}
& m+1, a \ldots\langle\ldots, j, \ldots ; a, \ldots|| \ldots, j, \ldots ; a, \ldots\rangle_{m+1, a \ldots} \widetilde{\lambda \rightarrow 0} \\
& \quad-\frac{8 \pi \mu^{2 \epsilon} \alpha_{\mathrm{s}}}{\lambda^{2}} \sum_{I \neq j} \frac{1}{p_{I} q} \sum_{K \neq j, I} m, a \ldots\left\langle\ldots ; a, \ldots\left|\boldsymbol{T}_{I} \cdot \boldsymbol{T}_{K}\left[\frac{p_{I} p_{K}}{\left(p_{I}+p_{K}\right) q}-\frac{m_{I}^{2}}{2 p_{I} q}\right]\right| \ldots ; a, \ldots\right\rangle_{m, a \ldots},
\end{aligned}
$$

where $|\ldots\rangle_{m, a \ldots}$ corresponds to the $m$-parton matrix element that results from the original $(m+1)$-parton matrix element upon omitting the gluon $j$, and the indices $I, K$ label both initial- and final-state partons. This formula is valid in the general case of massive or massless partons.

\subsection{Collinear and quasi-collinear limits}

In the massless case, the squared matrix element $\left|\mathcal{M}_{m+1}\right|^{2}$ diverges when two external partons become collinear [36]. If at least one of the external partons is massive, the collinear divergence is screened by the finite value of the parton mass. Nonetheless, the cross section contribution of the matrix element from this phase-space region is strongly (logarithmically) enhanced when the parton mass becomes small. As discussed in Sect. 2.1, to control these enhanced contributions we have to study the behaviour of the matrix element in the quasicollinear limit [31, 7]. Since the quasi-collinear limit is a generalization of the customary collinear limit, the latter can in turn be obtained as a special case from the former.

We consider two final-state partons $i$ and $j$ that can be produced by a QCD vertex through the splitting process $\tilde{i j} \rightarrow i+j$. The partons $i$ and $j$ have momenta $p_{i}$ and $p_{j}$ and masses $m_{i}$ and $m_{j}\left(p_{i}^{2}=m_{i}^{2}, p_{j}^{2}=m_{j}^{2}\right)$. The mass of the parton $\tilde{i j}$ is denoted by $m_{i j}$ and is constrained by $m_{i j} \leq m_{i}+m_{j}$. This constraint forbids the on-shell decay of the parton $\tilde{i j}$. We introduce the following Sudakov parametrization of the parton momenta:

$p_{i}^{\mu}=z p^{\mu}+k_{\perp}^{\mu}-\frac{k_{\perp}^{2}+z^{2} m_{i j}^{2}-m_{i}^{2}}{z} \frac{n^{\mu}}{2 p n}, \quad p_{j}^{\mu}=(1-z) p^{\mu}-k_{\perp}^{\mu}-\frac{k_{\perp}^{2}+(1-z)^{2} m_{i j}^{2}-m_{j}^{2}}{1-z} \frac{n^{\mu}}{2 p n}$,

where $p^{\mu}$ is a time-like momentum (with $p^{2}=m_{i j}^{2}$ ) that points towards the collinear (forward) direction, $n^{\mu}$ is an auxiliary light-like vector $\left(n^{2}=0\right)$ and $k_{\perp}$ is the momentum component that is orthogonal to both $p$ and $n\left(p k_{\perp}=n k_{\perp}=0\right)$. The invariant mass of the final-state partons is

$$
\left(p_{i}+p_{j}\right)^{2}=-\frac{k_{\perp}^{2}}{z(1-z)}+\frac{m_{i}^{2}}{z}+\frac{m_{j}^{2}}{1-z} .
$$

The quasi-collinear region is reached when $k_{\perp}$ becomes of $\mathrm{O}(m)$ and small. This region can be identified by performing the uniform rescaling

$$
k_{\perp} \rightarrow \lambda k_{\perp}, \quad m_{i} \rightarrow \lambda m_{i}, \quad m_{j} \rightarrow \lambda m_{j}, \quad m_{i j} \rightarrow \lambda m_{i j}
$$

and studying the limit $\lambda \rightarrow 0$. Neglecting terms that are less singular than $1 / \lambda^{2}$ the squared matrix element $\left|\mathcal{M}_{m+1}\right|^{2}$ behaves as 31]

$$
\begin{aligned}
& m+1, a \ldots\langle\ldots, i, j, \ldots ; a, \ldots|| \ldots, i, j, \ldots ; a, \ldots\rangle_{m+1, a \ldots} \widetilde{\lambda \rightarrow 0} \\
& \quad \frac{1}{\lambda^{2}} \frac{8 \pi \mu^{2 \epsilon} \alpha_{\mathrm{s}}}{\left(p_{i}+p_{j}\right)^{2}-m_{i j}^{2}} m, a \ldots\left\langle\ldots, \tilde{i j}, \ldots ; a, \ldots\left|\hat{P}_{\widetilde{i j}, i}\left(z, k_{\perp},\{m\} ; \epsilon\right)\right| \ldots, \tilde{i j}, \ldots ; a, \ldots\right\rangle_{m, a \ldots},
\end{aligned}
$$


where $|\ldots\rangle_{m, a \ldots}$ corresponds to the $m$-parton matrix element that is obtained from the $(m+1)$-parton matrix element by replacing the parton pair $i, j$ by the single parton $\tilde{i j}$.

The kernel $\hat{P}_{\widetilde{i j}, i}\left(z, k_{\perp},\{m\} ; \epsilon\right)$ in Eq. (4.5) is the generalization of the $d$-dimensional Altarelli-Parisi splitting function [36] from the collinear to the quasi-collinear limits. As in the collinear case, it depends on the momentum fraction $z$, on the transverse momentum $k_{\perp}$ and on the helicity of the parton $\tilde{i j}$ in the $m$-parton matrix element. In the quasi-collinear case, it also depends on the masses $m_{i}, m_{j}, m_{i j}$ of the partons involved in the splitting process. The mass dependence is shortly denoted by $\{m\}$.

The generalized Altarelli-Parisi kernels for the QCD splitting processes $Q \rightarrow Q+g$, $Q \rightarrow g+Q$ and $g \rightarrow Q+\bar{Q}$, are

$$
\begin{aligned}
& \left\langle s\left|\hat{P}_{Q Q}\left(z, k_{\perp}, m_{Q} ; \epsilon\right)\right| s^{\prime}\right\rangle=\delta_{s s^{\prime}} C_{\mathrm{F}}\left[\frac{1+z^{2}}{1-z}-\epsilon(1-z)-\frac{m_{Q}^{2}}{p_{Q} p_{g}}\right], \\
& \left\langle s\left|\hat{P}_{Q g}\left(z, k_{\perp}, m_{Q} ; \epsilon\right)\right| s^{\prime}\right\rangle=\delta_{s s^{\prime}} C_{\mathrm{F}}\left[\frac{1+(1-z)^{2}}{z}-\epsilon z-\frac{m_{Q}^{2}}{p_{g} p_{Q}}\right], \\
& \left\langle\mu\left|\hat{P}_{g Q}\left(z, k_{\perp}, m_{Q} ; \epsilon\right)\right| \nu\right\rangle=T_{\mathrm{R}}\left[-g^{\mu \nu}-4 \frac{k_{\perp}^{\mu} k_{\perp}^{\nu}}{\left(p_{Q}+p_{\bar{Q}}\right)^{2}}\right]
\end{aligned}
$$

where $s, s^{\prime}$ and $\mu, \nu$ denote the indices of the spin correlations for the parton $\tilde{i j}$, and the $k_{\perp}$ and mass dependence of the scalar products $p_{i} p_{j}$ is given in Eq. (4.3). Of course, the $g \rightarrow g g$ splitting function remains unaffected by the inclusion of mass terms,

$$
\left\langle\mu\left|\hat{P}_{g g}\left(z, k_{\perp} ; \epsilon\right)\right| \nu\right\rangle=2 C_{\mathrm{A}}\left[-g^{\mu \nu}\left(\frac{z}{1-z}+\frac{1-z}{z}\right)-2(1-\epsilon) z(1-z) \frac{k_{\perp}^{\mu} k_{\perp}^{\nu}}{k_{\perp}^{2}}\right] .
$$

We also need the spin-averaged form of the splitting functions. For quarks this is obtained by contraction with $\delta_{s s^{\prime}} / 2$ and for a gluon with on-shell momentum $p$, by contraction with

$$
\frac{1}{d-2} d_{\mu \nu}(p)=\frac{1}{2(1-\epsilon)}\left[-g_{\mu \nu}+(\text { gauge terms })\right]
$$

where the gauge terms are proportional either to $p^{\mu}$ or to $p^{\nu}$ and

$$
-g^{\mu \nu} d_{\mu \nu}(p)=d-2, \quad p^{\mu} d_{\mu \nu}(p)=0 .
$$

The spin-averaged QCD splitting functions only depend on $z, \epsilon$ and the ratio

$$
\mu_{i j}^{2}=\frac{m_{i}^{2}+m_{j}^{2}}{\left(p_{i}+p_{j}\right)^{2}-m_{i j}^{2}},
$$

and are denoted by $\left\langle\hat{P}_{\widetilde{i j}, i}\left(z ; \epsilon ; \mu_{i j}^{2}\right)\right\rangle$. They explicitly read

$$
\begin{aligned}
\left\langle\hat{P}_{Q Q}\left(z ; \epsilon ; \mu_{Q g}^{2}\right)\right\rangle & =C_{\mathrm{F}}\left[\frac{1+z^{2}}{1-z}-\epsilon(1-z)-2 \mu_{Q g}^{2}\right], \\
\left\langle\hat{P}_{Q g}\left(z ; \epsilon ; \mu_{g Q}^{2}\right)\right\rangle & =C_{\mathrm{F}}\left[\frac{1+(1-z)^{2}}{z}-\epsilon z-2 \mu_{g Q}^{2}\right], \\
\left\langle\hat{P}_{g Q}(z ; \epsilon)\right\rangle & =T_{\mathrm{R}}\left[1-\frac{2 z(1-z)-\mu_{Q \bar{Q}}^{2}}{1-\epsilon}\right] \\
\left\langle\hat{P}_{g g}(z ; \epsilon)\right\rangle & =2 C_{\mathrm{A}}\left[\frac{z}{1-z}+\frac{1-z}{z}+z(1-z)\right] .
\end{aligned}
$$


These generalized, spin-averaged splitting functions and some of their counterparts in SUSY QCD have already been given in Ref. [31]. The four-dimensional QED analogue of these functions describes the splitting processes $e^{ \pm} \rightarrow e^{ \pm}+\gamma, \gamma \rightarrow e^{+}+e^{-}$and is well known 46, 7, 25.

Setting $m_{i}=m_{j}=m_{i j}=0$ in all the expressions of this subsection, we recover the known expressions for the collinear limit (see e.g. Ref. [11]).

The matrix element $\mathcal{M}_{m+1, a \ldots}$ diverges also when a massless final-state parton $i$ becomes collinear to a massless initial-state parton $a$. The singularity is related to the splitting $a \rightarrow \widetilde{a i}+i$, where $\widetilde{a i}$ is the incoming parton of the related $m$-parton process. Since in this paper we restrict ourselves to massless incoming partons, none of the partons $a, i, \widetilde{a i}$ is massive and we can completely take over the factorization for massless partons described in Ref. [11]. There, the collinear limit is defined by $k_{\perp} \rightarrow 0$ in

$$
p_{i}^{\mu}=(1-x) p_{a}^{\mu}+k_{\perp}^{\mu}-\frac{k_{\perp}^{2}}{1-x} \frac{n^{\mu}}{2 p_{a} n}, \quad p_{a} p_{i}=-\frac{k_{\perp}^{2}}{2(1-x)}
$$

In this limit $\left|\mathcal{M}_{m+1}\right|^{2}$ behaves as

$$
\begin{aligned}
& m+1, a \ldots\langle\ldots, i, \ldots ; a, \ldots|| \ldots, i, \ldots ; a, \ldots\rangle_{m+1, a \ldots} \\
& \underset{k_{\perp \rightarrow 0}}{\sim} \frac{4 \pi \mu^{2 \epsilon} \alpha_{\mathrm{s}}}{x\left(p_{a} p_{i}\right)} \underset{m, \widetilde{a i} \ldots}{\sim}\left\langle\ldots ; \widetilde{a i}, \ldots\left|\hat{P}_{a, \widetilde{a i}}\left(x, k_{\perp} ; \epsilon\right)\right| \ldots ; \widetilde{a i}, \ldots\right\rangle_{m, \widetilde{a i} \ldots},
\end{aligned}
$$

where $|\ldots\rangle_{m, \tilde{a i} \ldots}$ corresponds to the $m$-parton matrix element that is obtained from the $(m+1)$-parton matrix element by replacing the parton pair $a, i$ by the single incoming parton $\widetilde{a i}$, which has the reduced momentum $x p_{a}$ with $0<x<1$.

\section{Dipole factorization formulae}

In the actual calculation of cross sections, Eqs. (4.1), (4.5) and (4.18) cannot straightforwardly be used as 'true' factorization formulae, because the momenta of the partons in the matrix elements on the right-hand sides are unambiguously defined only in the strict soft and (quasi-)collinear limits. In this section we define dipole factorization formulae that, besides having the correct limiting behaviour, implement momentum conservation away from the limits. Momentum conservation is implemented not only exactly but also in a factorizable way. The dipole factorization formulae can thus be used to introduce the dipole factors $\mathrm{d} V_{\text {dipole }}$ that are needed to construct (see Eq. (2.7)) the auxiliary cross section $\mathrm{d} \sigma^{\mathrm{A}}$ and its related integrated counterpart in Eq. (2.3).

We present the dipole factorization formulae as in Ref. [11:

$$
\left|\mathcal{M}_{m+1}\right|^{2}=\sum_{i, j} \sum_{k \neq i, j} \mathcal{D}_{i j, k}+\sum_{i, j} \sum_{a} \mathcal{D}_{i j}^{a}+\sum_{a, i} \sum_{j \neq i} \mathcal{D}_{j}^{a i}+\sum_{a, i} \sum_{b \neq a} \mathcal{D}^{a i, b}+\ldots
$$

where the terms on the right-hand side approximate the matrix element in different singular regions and the dots stand for terms that lead to finite integrals. Four different situations can be distinguished for the emitter-spectator pairs (see Fig. 1), since either one can 
$\underline{\mathcal{D}_{i j, k}}:$
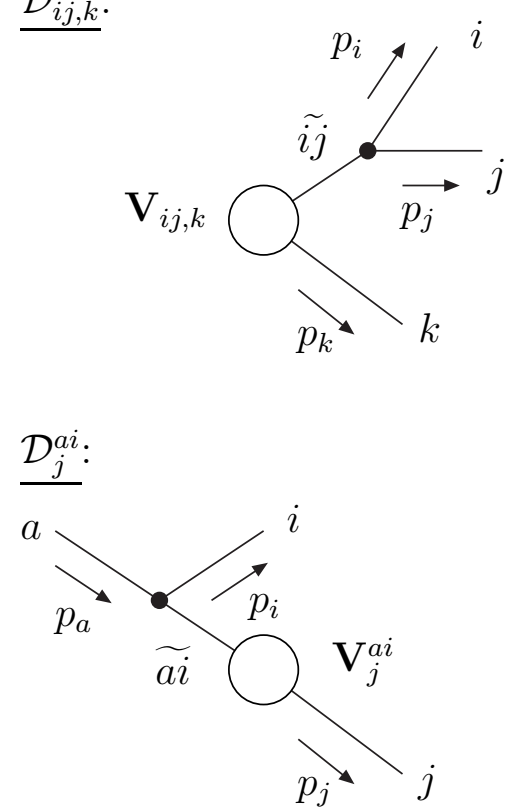

$\underline{\mathcal{D}_{i j}^{a}:}$

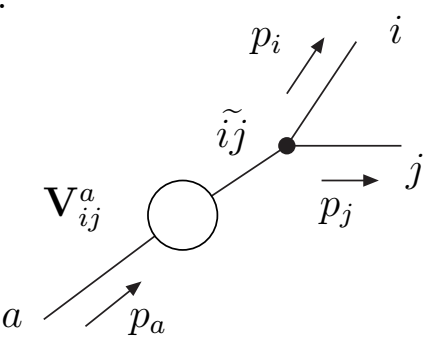

$\underline{\mathcal{D}^{a i, b}}:$

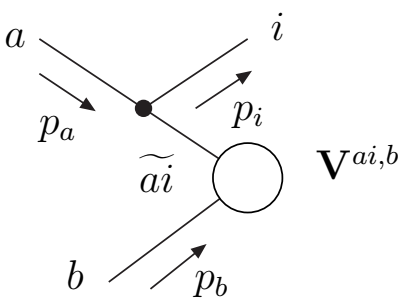

Figure 1: Effective diagrams for the different emitter-spectator cases.

belong to the final or initial states. The first two terms on the right-hand side control the singularities of the $(m+1)$-parton matrix element when two final-state partons $i$ and $j$ become (quasi-)collinear: the emitter is a final-state parton and the spectator can be either in the final $\left(\mathcal{D}_{i j, k}\right)$ or in the initial $\left(\mathcal{D}_{i j}^{a}\right)$ states. The third and fourth terms on the right-hand side control the singularities of the $(m+1)$-parton matrix element when a finalstate parton $i$ and an initial-state parton $a$ become collinear: the emitter is an initial-state parton and the spectator can be either in the final $\left(\mathcal{D}_{j}^{a i}\right)$ or in the initial $\left(\mathcal{D}^{a i, b}\right)$ states. When the parton $i$ is soft, all the four dipole functions $\mathcal{D}$ become singular. These four types of dipoles are considered in the following subsections.

The dipole factors introduced in Ref. [30] differ from ours in several respects. For instance, they do not deal with the case of massive partons with unequal masses and, typically, they treat the kinematic recoil differently. The main overall differences arise from the fact that they are not aimed to control the quasi-collinear region.

\subsection{Final-state emitter and final-state spectator}

The dipole contribution $\mathcal{D}_{i j, k}$ (see Fig. 1) to the factorization formula (5.1) is

$$
\begin{aligned}
& \mathcal{D}_{i j, k}\left(p_{1}, \ldots, p_{m+1}\right)= \\
& \quad-\frac{1}{\left(p_{i}+p_{j}\right)^{2}-m_{i j}^{2}}{ }_{m}\left\langle\ldots, \tilde{i j}, \ldots, \tilde{k}, \ldots\left|\frac{\boldsymbol{T}_{k} \cdot \boldsymbol{T}_{i j}}{\boldsymbol{T}_{i j}^{2}} \mathbf{V}_{i j, k}\right| \ldots, \tilde{i j}, \ldots, \tilde{k}, \ldots\right\rangle_{m}
\end{aligned}
$$

The final-state parton momenta $p_{i}, p_{j}$ and $p_{k}$ have arbitrary masses and their total outgoing momentum is denoted by $Q$,

$$
p_{i}^{2}=m_{i}^{2}, \quad p_{j}^{2}=m_{j}^{2}, \quad p_{k}^{2}=m_{k}^{2}, \quad Q=p_{i}+p_{j}+p_{k} .
$$


The $m$-parton matrix elements in Eq. (5.2) are obtained from the original $(m+1)$-parton matrix element by replacing $i$ and $j$ with the parent parton $\tilde{i j}$ in the splitting process $\tilde{i j} \rightarrow i+j$ and by changing the momentum of the spectator parton $k$. The on-shell mass of the emitter $\tilde{i j}$ is $m_{i j}$ and we only consider situations with $m_{i j} \leq m_{i}+m_{j}$. In Eq. (5.2) we have split off the colour structure from the spin functions $\mathbf{V}_{i j, k}$, which are given in Sect. 5.1.2.

\subsubsection{Kinematics and phase-space factorization}

The auxiliary momenta $\widetilde{p}_{i j}$ and $\widetilde{p}_{k}$ of the emitter and spectator are defined in such a way that $\widetilde{p}_{i j}$ plays the role of $p$ in the quasi-collinear limit (4.2), but at the same time they obey their mass-shell conditions and total momentum conservation,

$$
\widetilde{p}_{i j}^{2}=m_{i j}^{2}, \quad \widetilde{p}_{k}^{2}=m_{k}^{2}, \quad Q^{\mu}=\widetilde{p}_{i j}^{\mu}+\widetilde{p}_{k}^{\mu} .
$$

For later use, we introduce the rescaled parton masses $\mu_{n}$ and the relative velocities $v_{p, q}$ between two massive momenta $p^{\mu}$ and $q^{\mu}$,

$$
\mu_{n}=\frac{m_{n}}{\sqrt{Q^{2}}} \quad(n=i, j, k, i j), \quad v_{p, q}=\sqrt{1-\frac{p^{2} q^{2}}{(p q)^{2}}} .
$$

The relative velocities can also be written as

$$
v_{p, q}=\frac{\sqrt{\lambda\left((p+q)^{2}, p^{2}, q^{2}\right)}}{(p+q)^{2}-p^{2}-q^{2}}
$$

in terms of the customary triangular function $\lambda(x, y, z)$,

$$
\lambda(x, y, z)=x^{2}+y^{2}+z^{2}-2 x y-2 x z-2 y z .
$$

For instance, the velocity $\tilde{v}_{i j, k}$ between $\widetilde{p}_{i j}$ and $\widetilde{p}_{k}$ is given by

$$
\tilde{v}_{i j, k}=\frac{\sqrt{\lambda\left(1, \mu_{i j}^{2}, \mu_{k}^{2}\right)}}{1-\mu_{i j}^{2}-\mu_{k}^{2}}
$$

Our definition of the momenta $\widetilde{p}_{i j}$ and $\widetilde{p}_{k}$ in terms of the original momenta $p_{i}, p_{j}$ and $p_{k}\left(\right.$ or $\left.Q=p_{i}+p_{j}+p_{k}\right)$ is:

$$
\begin{aligned}
& \widetilde{p}_{k}^{\mu}=\frac{\sqrt{\lambda\left(Q^{2}, m_{i j}^{2}, m_{k}^{2}\right)}}{\sqrt{\lambda\left(Q^{2},\left(p_{i}+p_{j}\right)^{2}, m_{k}^{2}\right)}}\left(p_{k}^{\mu}-\frac{Q p_{k}}{Q^{2}} Q^{\mu}\right)+\frac{Q^{2}+m_{k}^{2}-m_{i j}^{2}}{2 Q^{2}} Q^{\mu}, \\
& \tilde{p}_{i j}^{\mu}=Q^{\mu}-\tilde{p}_{k}^{\mu}
\end{aligned}
$$

and it coincides with the one given in Ref. [25] for the specific case with $m_{i}=m_{i j}$ considered there. The definition in Eq. (5.9) is symmetric with respect to $i \leftrightarrow j$ and is uniformly applicable to any configuration of the parton masses $m_{n}(n=i, j, k, i j)$. Different definitions of the momenta $\widetilde{p}_{i j}$ and $\widetilde{p}_{k}$ can be introduced. The authors of Ref. [30] consider only 
the specific cases with $\left\{m_{i}=m_{j}=m_{i j}=0, m_{k} \neq 0\right\},\left\{m_{i}=m_{i j}, m_{k}=m_{j}=0\right\}$ and $\left\{m_{i}=m_{i j}=m_{k}, m_{j}=0\right\}$ : in the last two cases their definition coincides with ours.

Next we exactly factorize the three-particle phase space $\mathrm{d} \phi\left(p_{i}, p_{j}, p_{k} ; Q\right)$ of Eq. (3.7) in terms of the two-particle phase space $\mathrm{d} \phi\left(\widetilde{p}_{i j}, \widetilde{p}_{k} ; Q\right)$ and a single-particle phase-space factor $\left[\mathrm{d} p_{i}\left(\widetilde{p}_{i j}, \widetilde{p}_{k}\right)\right]$,

$$
\mathrm{d} \phi\left(p_{i}, p_{j}, p_{k} ; Q\right)=\mathrm{d} \phi\left(\widetilde{p}_{i j}, \widetilde{p}_{k} ; Q\right)\left[\mathrm{d} p_{i}\left(\widetilde{p}_{i j}, \widetilde{p}_{k}\right)\right] \Theta\left(1-\mu_{i}-\mu_{j}-\mu_{k}\right) .
$$

In App. B of Ref. 25] the derivation of this phase-space splitting was outlined for the case with $m_{i}=m_{i j}$ in four space-time dimensions. The generalization to $m_{i} \neq m_{i j}$ in $d$ dimensions is straightforward; we present only the results. We obtain

$$
\begin{aligned}
\int\left[\mathrm{d} p_{i}\left(\widetilde{p}_{i j}, \widetilde{p}_{k}\right)\right]= & \frac{1}{4}(2 \pi)^{-3+2 \epsilon}\left(Q^{2}\right)^{1-\epsilon}\left(1-\mu_{i}^{2}-\mu_{j}^{2}-\mu_{k}^{2}\right)^{2-2 \epsilon}\left[\lambda\left(1, \mu_{i j}^{2}, \mu_{k}^{2}\right)\right]^{\frac{-1+2 \epsilon}{2}} \int \mathrm{d}^{d-3} \Omega \\
& \times \int_{y_{-}}^{y_{+}} \mathrm{d} y_{i j, k}\left(1-y_{i j, k}\right)^{1-2 \epsilon}\left[\mu_{i}^{2}+\mu_{j}^{2}+\left(1-\mu_{i}^{2}-\mu_{j}^{2}-\mu_{k}^{2}\right) y_{i j, k}\right]^{-\epsilon} \\
& \times \int_{z_{-}\left(y_{i j, k}\right)}^{z_{+}\left(y_{i j, k}\right)} \mathrm{d} \tilde{z}_{i}\left[z_{+}\left(y_{i j, k}\right)-\tilde{z}_{i}\right]^{-\epsilon}\left[\tilde{z}_{i}-z_{-}\left(y_{i j, k}\right)\right]^{-\epsilon}
\end{aligned}
$$

where the variables $\tilde{z}_{i}$ and $y_{i j, k}$ are defined as in Refs. [11, 25]:

$$
\tilde{z}_{i}=1-\tilde{z}_{j}=\frac{p_{i} p_{k}}{p_{i} p_{k}+p_{j} p_{k}}, \quad y_{i j, k}=\frac{p_{i} p_{j}}{p_{i} p_{j}+p_{i} p_{k}+p_{j} p_{k}} .
$$

Their integration boundary is given by

$$
\begin{aligned}
y_{-} & =\frac{2 \mu_{i} \mu_{j}}{1-\mu_{i}^{2}-\mu_{j}^{2}-\mu_{k}^{2}}, \quad y_{+}=1-\frac{2 \mu_{k}\left(1-\mu_{k}\right)}{1-\mu_{i}^{2}-\mu_{j}^{2}-\mu_{k}^{2}}, \\
z_{ \pm}\left(y_{i j, k}\right) & =\frac{2 \mu_{i}^{2}+\left(1-\mu_{i}^{2}-\mu_{j}^{2}-\mu_{k}^{2}\right) y_{i j, k}}{2\left[\mu_{i}^{2}+\mu_{j}^{2}+\left(1-\mu_{i}^{2}-\mu_{j}^{2}-\mu_{k}^{2}\right) y_{i j, k}\right]}\left(1 \pm v_{i j, i} v_{i j, k}\right),
\end{aligned}
$$

where $v_{i j, i}\left(v_{i j, k}\right)$ is the relative velocity between $p_{i}+p_{j}$ and $p_{i}\left(p_{k}\right)$. The relative velocities are functions of $y_{i j, k}$ and explicitly read

$$
\begin{aligned}
& v_{i j, k}= \frac{\sqrt{\left[2 \mu_{k}^{2}+\left(1-\mu_{i}^{2}-\mu_{j}^{2}-\mu_{k}^{2}\right)\left(1-y_{i j, k}\right)\right]^{2}-4 \mu_{k}^{2}}}{\left(1-\mu_{i}^{2}-\mu_{j}^{2}-\mu_{k}^{2}\right)\left(1-y_{i j, k}\right)}, \\
& v_{i j, i}=\frac{\sqrt{\left(1-\mu_{i}^{2}-\mu_{j}^{2}-\mu_{k}^{2}\right)^{2} y_{i j, k}^{2}-4 \mu_{i}^{2} \mu_{j}^{2}}}{\left(1-\mu_{i}^{2}-\mu_{j}^{2}-\mu_{k}^{2}\right) y_{i j, k}+2 \mu_{i}^{2}} .
\end{aligned}
$$

The $\mathrm{d}^{d-3} \Omega$ integration extends over the solid angle perpendicular to $\widetilde{p}_{i j}$ and $\widetilde{p}_{k}$ and thus

$$
\int \mathrm{d}^{d-3} \Omega=\frac{2 \pi}{\pi^{\epsilon} \Gamma(1-\epsilon)}
$$




\subsubsection{The dipole splitting functions}

We give the functions $\mathbf{V}_{i j, k}$ in Eq. (5.2) for the three QCD splitting processes $\tilde{i j} \rightarrow i+j$ :

- $Q \rightarrow g\left(p_{i}\right)+Q\left(p_{j}\right): \quad m_{i}=0$ and $m_{j}=m_{i j}=m_{Q}$,

- $g \rightarrow Q\left(p_{i}\right)+\bar{Q}\left(p_{j}\right): \quad m_{i}=m_{j}=m_{Q}$ and $m_{i j}=0$,

- $g \rightarrow g\left(p_{i}\right)+g\left(p_{j}\right): \quad m_{i}=m_{j}=m_{i j}=0$.

The case $\bar{Q} \rightarrow g \bar{Q}$ is formally identical to $Q \rightarrow g Q$. The spectator mass $m_{k}$ may be zero or non-zero in all cases. Note that in general $\mathbf{V}_{i j, k}$ is non-diagonal in the helicity space of the parton $\tilde{i j}$. For the analytical integration over the singular degrees of freedom (see Sect. 5.1.3) we also need the spin-averaged functions $\left\langle\mathbf{V}_{i j, k}\right\rangle$. Denoting $s, s^{\prime}$ or $\mu, \nu$ the helicities of $\tilde{i j}=Q$ or $\tilde{i j}=g$ in $\langle\ldots, \widetilde{i j}, \ldots \| \ldots, \widetilde{i j}, \ldots\rangle$, we define the dipole functions $\mathbf{V}_{i j, k}$ as follows:

$$
\begin{aligned}
& \left\langle s\left|\mathbf{V}_{g Q, k}\right| s^{\prime}\right\rangle=8 \pi \mu^{2 \epsilon} \alpha_{\mathrm{s}} C_{\mathrm{F}}\left\{\frac{2}{1-\tilde{z}_{j}\left(1-y_{i j, k}\right)}-\frac{\tilde{v}_{i j, k}}{v_{i j, k}}\left[1+\tilde{z}_{j}+\frac{m_{Q}^{2}}{p_{i} p_{j}}+\epsilon\left(1-\tilde{z}_{j}\right)\right]\right\} \delta_{s s^{\prime}} \\
& =\left\langle\mathbf{V}_{g Q, k}\right\rangle \delta_{s s^{\prime}} \\
& \left\langle\mu\left|\mathbf{V}_{Q \bar{Q}, k}\right| \nu\right\rangle=8 \pi \mu^{2 \epsilon} \alpha_{\mathrm{s}} T_{\mathrm{R}} \frac{1}{v_{i j, k}}\left\{-g^{\mu \nu}\left[1-\frac{2 \kappa}{1-\epsilon}\left(z_{+} z_{-}-\frac{m_{Q}^{2}}{\left(p_{i}+p_{j}\right)^{2}}\right)\right]\right. \\
& \left.-\frac{4}{\left(p_{i}+p_{j}\right)^{2}}\left[\tilde{z}_{i}^{(m)} p_{i}^{\mu}-\tilde{z}_{j}^{(m)} p_{j}^{\mu}\right]\left[\tilde{z}_{i}^{(m)} p_{i}^{\nu}-\tilde{z}_{j}^{(m)} p_{j}^{\nu}\right]\right\} \\
& \left\langle\mathbf{V}_{Q \bar{Q}, k}\right\rangle=8 \pi \mu^{2 \epsilon} \alpha_{\mathrm{s}} T_{\mathrm{R}} \frac{1}{v_{i j, k}}\left\{1-\frac{2}{1-\epsilon}\left[\tilde{z}_{i}\left(1-\tilde{z}_{i}\right)-(1-\kappa) z_{+} z_{-}\right.\right. \\
& \left.\left.-\frac{\kappa \mu_{Q}^{2}}{2 \mu_{Q}^{2}+\left(1-2 \mu_{Q}^{2}-\mu_{k}^{2}\right) y_{i j, k}}\right]\right\} \\
& \left\langle\mu\left|\mathbf{V}_{g g, k}\right| \nu\right\rangle=16 \pi \mu^{2 \epsilon} \alpha_{\mathrm{s}} C_{\mathrm{A}}\left\{-g^{\mu \nu}\left[\frac{1}{1-\tilde{z}_{i}\left(1-y_{i j, k}\right)}+\frac{1}{1-\tilde{z}_{j}\left(1-y_{i j, k}\right)}-\frac{2-\kappa z_{+} z_{-}}{v_{i j, k}}\right]\right. \\
& \left.+\frac{1}{v_{i j, k}} \frac{1-\epsilon}{p_{i} p_{j}}\left[\tilde{z}_{i}^{(m)} p_{i}^{\mu}-\tilde{z}_{j}^{(m)} p_{j}^{\mu}\right]\left[\tilde{z}_{i}^{(m)} p_{i}^{\nu}-\tilde{z}_{j}^{(m)} p_{j}^{\nu}\right]\right\} \\
& \left\langle\mathbf{V}_{g g, k}\right\rangle=16 \pi \mu^{2 \epsilon} \alpha_{\mathrm{s}} C_{\mathrm{A}}\left\{\frac{1}{1-\tilde{z}_{i}\left(1-y_{i j, k}\right)}+\frac{1}{1-\tilde{z}_{j}\left(1-y_{i j, k}\right)}\right. \\
& \left.+\frac{\tilde{z}_{i}\left(1-\tilde{z}_{i}\right)-(1-\kappa) z_{+} z_{-}-2}{v_{i j, k}}\right\},
\end{aligned}
$$

where $z_{ \pm}$is given in Eq. (5.13) and the new variables $\tilde{z}_{i}^{(m)}$ and $\tilde{z}_{j}^{(m)}$ are

$$
\tilde{z}_{i}^{(m)}=\tilde{z}_{i}-\frac{1}{2}\left(1-v_{i j, k}\right), \quad \tilde{z}_{j}^{(m)}=\tilde{z}_{j}-\frac{1}{2}\left(1-v_{i j, k}\right) .
$$

The constant $\kappa$ is a free parameter, which only redistributes non-singular contributions between the different terms within square brackets in Eq. (2.3). Choosing different values 
of $\kappa$, we can simplify the expressions of either the subtraction term $(\kappa=0)$ or its integral $(\kappa=2 / 3)$. Of course, the final result for $\sigma^{\mathrm{NLO}}$ must not depend on $\kappa$, i.e. its $\kappa$-independence can serve as a consistency check of the calculation. The velocity factors $\tilde{v}_{i j, k} / v_{i j, k}$ and $1 / v_{i j, k}$ are also harmless in the singular limits; they are introduced in the definition of the functions $\mathbf{V}_{i j, k}$ only to simplify their integration in analytic form. The definition of the four-dimensional $\mathbf{V}_{g Q, k}$ in Ref. [25] differs from ours by some harmless velocity factors.

In App. C, we give the dipole splitting functions $\mathbf{V}_{i j, k}$ that are relevant for SUSY QCD calculations.

\subsubsection{The integrated dipole functions}

The spin-correlation terms of the splitting functions in Eqs. (5.17) and (5.19) have been

defined such that $\left[\tilde{z}_{i}^{(m)} p_{i}^{\mu}-\tilde{z}_{j}^{(m)} p_{j}^{\mu}\right] \tilde{p}_{i j, \mu}=0$, when $m_{i}=m_{j}, m_{i j}=0$. This property ensures [11] that the spin correlations vanishes after azimuthal integration. In the evaluation of the integrals of the dipole factors over the phase space $\left[\mathrm{d} p_{i}\left(\widetilde{p}_{i j}, \widetilde{p}_{k}\right)\right]$, we can thus replace the spin matrices $\mathbf{V}_{i j, k}$ with their spin averages $\left\langle\mathbf{V}_{i j, k}\right\rangle$. We define

$$
\int\left[\mathrm{d} p_{i}\left(\widetilde{p}_{i j}, \widetilde{p}_{k}\right)\right] \frac{1}{\left(p_{i}+p_{j}\right)^{2}-m_{i j}^{2}}\left\langle\mathbf{V}_{i j, k}\right\rangle \equiv \frac{\alpha_{\mathrm{s}}}{2 \pi} \frac{1}{\Gamma(1-\epsilon)}\left(\frac{4 \pi \mu^{2}}{Q^{2}}\right)^{\epsilon} I_{i j, k}(\epsilon)
$$

where $I_{i j, k}(\epsilon)$ depends also on the parton masses and $\widetilde{p}_{i j} \cdot \widetilde{p}_{k}$. In the massless case, the integrals $I_{i j, k}(\epsilon)$ do not depend on $\widetilde{p}_{i j} \cdot \widetilde{p}_{k}$ and become the functions $\mathcal{V}_{i j}(\epsilon)$ of Ref. [11]. For non-vanishing values of the parton masses, these integrals cannot be exactly performed in $d$ dimensions in terms of simple functions. We evaluate them by neglecting corrections of $\mathrm{O}(\epsilon)$. Note, however, that we want to compute them for arbitrary (finite or vanishing) masses, so we do not want to spoil the commutativity of the massless limit with the limit $\epsilon \rightarrow 0$. We thus perform the $\epsilon$ expansion uniformly in the parton masses, i.e. the coefficients of the $\mathrm{O}(\epsilon)$ corrections that we neglect are not singular when one or more parton masses vanish. For instance, we do not expand in $\epsilon$ terms of the form $\mu_{j}^{-2 \epsilon}$, whose limits $\mu_{j} \rightarrow 0$ and $\epsilon \rightarrow 0$ do not commute.

We decompose the integrals $I_{i j, k}(\epsilon)$ into an eikonal part, $I^{\text {eik }}(\epsilon)$, which contains the soft integrals, and a remaining collinear part, $I_{i j, k}^{\text {coll }}(\epsilon)$ :

$$
\begin{aligned}
I_{g Q, k}\left(\mu_{Q}, \mu_{k} ; \epsilon\right) & =C_{\mathrm{F}}\left[2 I^{\mathrm{eik}}\left(\mu_{Q}, \mu_{k} ; \epsilon\right)+I_{g Q, k}^{\mathrm{coll}}\left(\mu_{Q}, \mu_{k} ; \epsilon\right)\right], \\
I_{Q \bar{Q}, k}\left(\mu_{Q}, \mu_{k} ; \epsilon\right) & =T_{\mathrm{R}} I_{Q \bar{Q}, k}^{\mathrm{coll}}\left(\mu_{Q}, \mu_{k} ; \epsilon\right) \\
I_{g g, k}\left(\mu_{k} ; \epsilon\right) & =2 C_{\mathrm{A}}\left[2 I^{\mathrm{eik}}\left(0, \mu_{k} ; \epsilon\right)+I_{g g, k}^{\mathrm{coll}}\left(\mu_{k} ; \epsilon\right)\right]
\end{aligned}
$$

The eikonal integral is defined by

$$
\frac{\alpha_{\mathrm{s}}}{2 \pi} \frac{1}{\Gamma(1-\epsilon)}\left(\frac{4 \pi \mu^{2}}{Q^{2}}\right)^{\epsilon} I^{\mathrm{eik}}\left(\mu_{j}, \mu_{k} ; \epsilon\right)=\int\left[\mathrm{d} p_{i}\left(\widetilde{p}_{i j}, \widetilde{p}_{k}\right)\right] \frac{1}{2 p_{i} p_{j}} \frac{8 \pi \mu^{2 \epsilon} \alpha_{\mathrm{s}}}{1-\tilde{z}_{j}\left(1-y_{i j, k}\right)},
$$

where $m_{i}=0$ and $m_{i j}=m_{j}$ in the kinematics defined in Sect. 5.1.2. This integral can be obtained as a complicated expression of dilogarithmic functions (see App. A). However, for 
the calculation of physical cross sections (see Sect. 6), we explicitly need only the symmetric part of $I^{\text {eik }}$ and that is simpler. We define

$$
I_{ \pm}^{\mathrm{eik}}\left(\mu_{j}, \mu_{k} ; \epsilon\right)=\frac{1}{2}\left[I^{\mathrm{eik}}\left(\mu_{j}, \mu_{k} ; \epsilon\right) \pm I^{\mathrm{eik}}\left(\mu_{k}, \mu_{j} ; \epsilon\right)\right]
$$

so that

$$
I^{\mathrm{eik}}\left(\mu_{j}, \mu_{k} ; \epsilon\right)=I_{+}^{\mathrm{eik}}\left(\mu_{j}, \mu_{k} ; \epsilon\right)+I_{-}^{\mathrm{eik}}\left(\mu_{j}, \mu_{k} ; \epsilon\right) .
$$

The symmetric part of the eikonal integral is given by

$$
\begin{aligned}
I_{+}^{\mathrm{eik}}\left(\mu_{j}, \mu_{k} ; \epsilon\right)= & \frac{1}{\tilde{v}_{i j, k}}\left[\left(1-\left(\mu_{j}+\mu_{k}\right)^{2}\right)^{-2 \epsilon} \frac{1}{2 \epsilon^{2}}\left(1-\frac{1}{2} \rho_{j}^{-2 \epsilon}-\frac{1}{2} \rho_{k}^{-2 \epsilon}\right)\right. \\
& +\frac{\pi^{2}}{24}\left(6-\mu_{j}^{-2 \epsilon}-\mu_{k}^{-2 \epsilon}\right)+2 \operatorname{Li}_{2}(-\rho)-2 \operatorname{Li}_{2}(1-\rho) \\
& \left.-\frac{1}{2} \operatorname{Li}_{2}\left(1-\rho_{j}^{2}\right)-\frac{1}{2} \operatorname{Li}_{2}\left(1-\rho_{k}^{2}\right)\right]+\mathrm{O}(\epsilon)
\end{aligned}
$$

where

$$
\begin{aligned}
\rho_{n}\left(\mu_{j}, \mu_{k}\right) & =\sqrt{\frac{1-\tilde{v}_{i j, k}+2 \mu_{n}^{2} /\left(1-\mu_{j}^{2}-\mu_{k}^{2}\right)}{1+\tilde{v}_{i j, k}+2 \mu_{n}^{2} /\left(1-\mu_{j}^{2}-\mu_{k}^{2}\right)}} \quad(n=j, k), \\
\rho & =\sqrt{\frac{1-\tilde{v}_{i j, k}}{1+\tilde{v}_{i j, k}}} .
\end{aligned}
$$

For the special case $\mu_{j}=0$, which is needed for the $g \rightarrow g g$ splitting, the result for $I_{+}^{\text {eik }}\left(0, \mu_{k} ; \epsilon\right)$ can be easily read off. Using $\rho_{j}\left(0, \mu_{k}\right)=0, \rho_{k}\left(0, \mu_{k}\right)=\mu_{k}$ and $\rho=0$, we obtain

$$
I_{+}^{\mathrm{eik}}\left(0, \mu_{k} ; \epsilon\right)=\left(1-\mu_{k}^{2}\right)^{-2 \epsilon} \frac{1}{2 \epsilon^{2}}\left(1-\frac{1}{2} \mu_{k}^{-2 \epsilon}\right)-\frac{\pi^{2}}{6}-\frac{\pi^{2}}{24} \mu_{k}^{-2 \epsilon}-\frac{1}{2} \operatorname{Li}_{2}\left(1-\mu_{k}^{2}\right)+\mathrm{O}(\epsilon) .
$$

If both masses vanish, then $I_{-}^{\mathrm{eik}}(0,0)=0$, and we recover the massless result [1]:

$$
I^{\mathrm{eik}}(0,0 ; \epsilon)=I_{+}^{\mathrm{eik}}(0,0 ; \epsilon)=\frac{1}{2 \epsilon^{2}}-\frac{\pi^{2}}{4}+\mathrm{O}(\epsilon) .
$$

For the case of non-vanishing masses, we have $\rho_{j} \rho_{k}=\rho$, therefore, we can use the relation

$$
1-\frac{1}{2} \rho_{j}^{-2 \epsilon}-\frac{1}{2} \rho_{k}^{-2 \epsilon}=\epsilon \ln \rho-\epsilon^{2}\left(\ln ^{2} \rho_{j}+\ln ^{2} \rho_{k}\right)+\mathrm{O}\left(\epsilon^{3}\right)
$$

to obtain the following expansion in $\epsilon$ :

$$
\begin{aligned}
& I_{+}^{\mathrm{eik}}\left(\mu_{j}, \mu_{k} ; \epsilon\right)= \\
& \frac{1}{\tilde{v}_{i j, k}}\left[\frac{1}{2 \epsilon} \ln \rho-\ln \rho \ln \left(1-\left(\mu_{j}+\mu_{k}\right)^{2}\right)-\frac{1}{2} \ln ^{2} \rho_{j}-\frac{1}{2} \ln ^{2} \rho_{k}\right. \\
& \left.\quad+\frac{\pi^{2}}{6}+2 \operatorname{Li}_{2}(-\rho)-2 \mathrm{Li}_{2}(1-\rho)-\frac{1}{2} \operatorname{Li}_{2}\left(1-\rho_{j}^{2}\right)-\frac{1}{2} \operatorname{Li}_{2}\left(1-\rho_{k}^{2}\right)\right] \\
& \quad+\mathrm{O}(\epsilon) .
\end{aligned}
$$


The integrals of the collinear parts are simpler. The explicit results are

$$
\begin{aligned}
I_{g Q, k}^{\mathrm{coll}}\left(\mu_{Q}, \mu_{k} ; \epsilon\right)= & \frac{3}{2 \epsilon}-\frac{\mu_{Q}^{-2 \epsilon}}{2 \epsilon}-2 \mu_{Q}^{-2 \epsilon}-2 \ln \left[\left(1-\mu_{k}\right)^{2}-\mu_{Q}^{2}\right]+\ln \left(1-\mu_{k}\right) \\
& -\frac{2 \mu_{Q}^{2}}{1-\mu_{Q}^{2}-\mu_{k}^{2}} \ln \left(\frac{\mu_{Q}}{1-\mu_{k}}\right)+5-\frac{\mu_{k}}{1-\mu_{k}}-\frac{2 \mu_{k}\left(1-2 \mu_{k}\right)}{1-\mu_{Q}^{2}-\mu_{k}^{2}} \\
& +\mathrm{O}(\epsilon), \\
I_{Q \bar{Q}, k}^{\text {coll }}\left(\mu_{Q}, \mu_{k} ; \epsilon\right)= & -\frac{2}{3}\left[\frac{1}{\epsilon}\left(1-\mu_{Q}^{-2 \epsilon}\right)-2 \ln \left(1-\mu_{k}\right)-2 \ln \left(\frac{1+\rho_{1}}{2}\right)+\frac{2}{3} \rho_{1}\left(3+\rho_{1}^{2}\right)-\frac{2 \rho_{1}^{3} \mu_{k}}{1+\mu_{k}}\right] \\
& +\left(\kappa-\frac{2}{3}\right) \frac{2 \mu_{k}^{2}}{1-\mu_{k}^{2}}\left[\rho_{2}^{3} \ln \left(\frac{\rho_{2}-\rho_{1}}{\rho_{2}+\rho_{1}}\right)-\ln \left(\frac{1-\rho_{1}}{1+\rho_{1}}\right)-\frac{8 \rho_{1} \mu_{Q}^{2}}{1-\mu_{k}^{2}}\right] \\
& +\mathrm{O}(\epsilon), \\
& \frac{11}{6 \epsilon}+\frac{50}{9}-\frac{11}{3}\left[\frac{\mu_{k}}{1+\mu_{k}}+\ln \left(1-\mu_{k}\right)\right]+\frac{(2-3 \kappa) \mu_{k}^{2}}{3\left(1-\mu_{k}^{2}\right)} \ln \left(\frac{2 \mu_{k}}{1+\mu_{k}}\right) \\
& +\mathrm{O}(\epsilon),
\end{aligned}
$$

with

$$
\rho_{1}=\sqrt{1-\frac{4 \mu_{Q}^{2}}{\left(1-\mu_{k}\right)^{2}}}, \quad \rho_{2}=\sqrt{1-\frac{4 \mu_{Q}^{2}}{1-\mu_{k}^{2}}} .
$$

Finally, we inspect the special case of parametrically-small masses which is relevant if one considers massless fermions and collinear singularities regularized by small fermion masses, as is often done in electroweak physics. The corresponding asymptotic forms of $I_{+}^{\text {eik }}$ and $I_{i j, k}^{\text {coll }}$ in this limit read

$$
\begin{aligned}
& I_{+}^{\mathrm{eik}} \underset{m_{j}, m_{k} \rightarrow 0}{\widetilde{4}} \frac{1}{4 \epsilon} \ln \frac{m_{j}^{2}}{s_{i j, k}}+\frac{1}{4 \epsilon} \ln \frac{m_{k}^{2}}{s_{i j, k}}-\frac{1}{8} \ln ^{2} \frac{m_{j}^{2}}{s_{i j, k}}-\frac{1}{8} \ln ^{2} \frac{m_{k}^{2}}{s_{i j, k}}-\frac{\pi^{2}}{3}, \\
& I_{g Q, k}^{\mathrm{coll}} \underset{m_{Q}, \widetilde{m_{k}} \rightarrow 0}{\frac{1}{\epsilon}}+\frac{1}{2} \ln \frac{m_{Q}^{2}}{s_{i j, k}}+3, \\
& I_{Q \bar{Q}, k}^{\text {coll }} \quad \underset{m_{Q}, m_{k} \rightarrow 0}{\longrightarrow}-\frac{2}{3} \ln \frac{m_{Q}^{2}}{s_{i j, k}}-\frac{16}{9}, \\
& I_{g g, k}^{\text {coll }} \underset{m_{k} \rightarrow 0}{\sim} \frac{11}{6 \epsilon}+\frac{50}{9},
\end{aligned}
$$

where $s_{i j, k}=2 \widetilde{p}_{i j} \widetilde{p}_{k}$. Note that in these expressions the fermion mass is only regarded as collinear regulator. Therefore, on the right-hand side we have neglected not only contributions of $\mathrm{O}(\epsilon)$, but also all the contributions of $\mathrm{O}\left(\mathrm{m}^{2} / s_{i j, k}\right)$, even in coefficients of $\epsilon$ poles. The collinear logarithmic contributions in the $Q \rightarrow g Q$ splitting are related to those in the fermion-photon splitting considered in Ref. [25], where soft divergences were regularized by an infinitesimal photon mass. 


\subsection{Final-state emitter and initial-state spectator}

The dipole contribution $\mathcal{D}_{i j}^{a}$ (see Fig. 1) to the factorization formula (5.1) is defined in close analogy to that in Eq. (5.2):

$$
\begin{aligned}
& \mathcal{D}_{i j}^{a}\left(p_{1}, \ldots, p_{m+1} ; p_{a}, \ldots\right)= \\
& \quad-\frac{1}{\left(p_{i}+p_{j}\right)^{2}-m_{i j}^{2}} \frac{1}{x_{i j, a}}{ }_{m, a}\left\langle\ldots, \tilde{i j}, \ldots ; \tilde{a}, \ldots\left|\frac{\boldsymbol{T}_{a} \cdot \boldsymbol{T}_{i j}}{\boldsymbol{T}_{i j}^{2}} \mathbf{V}_{i j}^{a}\right| \ldots, \tilde{i j}, \ldots ; \tilde{a}, \ldots\right\rangle_{m, a} .
\end{aligned}
$$

The $m$-parton matrix elements on the right-hand side are obtained from the original $(m+1)$ parton matrix element by replacing the final-state partons $i$ and $j$ with the emitter parton $\widetilde{i j}$ and the initial-state parton $a$ with the spectator parton $\tilde{a}$.

The final-state parton momenta $p_{i}$ and $p_{j}$ have arbitrary masses, the initial-state momentum $p_{a}$ is massless and their total transferred momentum is denoted by $Q$ (not to be confused with $Q$ in Eq. (5.3)),

$$
p_{i}^{2}=m_{i}^{2}, \quad p_{j}^{2}=m_{j}^{2}, \quad p_{a}^{2}=0, \quad Q=p_{i}+p_{j}-p_{a} .
$$

The on-shell mass of the emitter is $m_{i j}$, with $m_{i j} \leq m_{i}+m_{j}$.

As discussed in Sect. 2.2, we do not consider massive partons in the initial state in this paper. A treatment of massive initial-state particles is described in Ref. [25] for photon emission off fermions.

\subsubsection{Kinematics and phase-space factorization}

We introduce the auxiliary variables

$$
\begin{aligned}
x_{i j, a} & =\frac{p_{a} p_{i}+p_{a} p_{j}-p_{i} p_{j}+\frac{1}{2}\left(m_{i j}^{2}-m_{i}^{2}-m_{j}^{2}\right)}{p_{a} p_{i}+p_{a} p_{j}}, \\
\tilde{z}_{i} & =\frac{p_{a} p_{i}}{p_{a} p_{i}+p_{a} p_{j}}, \quad \tilde{z}_{j}=\frac{p_{a} p_{j}}{p_{a} p_{i}+p_{a} p_{j}}
\end{aligned}
$$

and we define the momenta of the emitter and spectator as

$$
\tilde{p}_{a}^{\mu}=x_{i j, a} p_{a}^{\mu}, \quad \tilde{p}_{i j}^{\mu}=p_{i}^{\mu}+p_{j}^{\mu}-\left(1-x_{i j, a}\right) p_{a}^{\mu} .
$$

These momenta obey their mass-shell relations and momentum conservation,

$$
\widetilde{p}_{i j}^{2}=m_{i j}^{2}, \quad \tilde{p}_{a}^{2}=0, \quad Q=\widetilde{p}_{i j}-\tilde{p}_{a} .
$$

We also define the rescaled parton masses $\mu_{n}$ as

$$
\mu_{n}=\frac{m_{n}}{\sqrt{2 \widetilde{p}_{i j} p_{a}}}, \quad n=i, j, i j .
$$

The momentum definition in Eq. (5.43) coincides with that of Ref. [30] for the specific case (with $m_{i j}=m_{j}$ and $m_{i}=0$ ) considered there and with Ref. 225 for $m_{i j}=m_{i}$ and $m_{j}=0$, but differs for arbitrary combinations of masses. 
Since the introduction of the rescaled momentum $\tilde{p}_{a}=x_{i j, a} p_{a}$ defines a new (boosted) frame, the factorization of the two-particle phase space $\mathrm{d} \phi\left(\widetilde{p}_{i j} ; Q+\tilde{p}_{a}\right)$ from the threeparticle phase space $\mathrm{d} \phi\left(p_{i}, p_{j} ; Q+p_{a}\right)$ involves a convolution over a boost parameter $x$,

$$
\mathrm{d} \phi\left(p_{i}, p_{j} ; Q+p_{a}\right)=\int_{0}^{1} \mathrm{~d} x \mathrm{~d} \phi\left(\widetilde{p}_{i j} ; Q+x p_{a}\right)\left[\mathrm{d} p_{i}\left(\widetilde{p}_{i j} ; p_{a}, x\right)\right] \Theta\left(x_{+}-x\right),
$$

with the upper limit

$$
x_{+}=1+\mu_{i j}^{2}-\left(\mu_{i}+\mu_{j}\right)^{2} .
$$

The single-parton phase space $\left[\mathrm{d} p_{i}\left(\widetilde{p}_{i j} ; p_{a}, x\right)\right]$ is

$$
\begin{aligned}
\int\left[\mathrm{d} p_{i}\left(\widetilde{p}_{i j} ; p_{a}, x\right)\right] & =\frac{1}{4}(2 \pi)^{-3+2 \epsilon}\left(2 \widetilde{p}_{i j} p_{a}\right)^{1-\epsilon} \int_{0}^{x_{+}} \mathrm{d} x_{i j, a} \delta\left(x-x_{i j, a}\right)\left(1-x+\mu_{i j}^{2}\right)^{-\epsilon} \\
& \times \int \mathrm{d}^{d-3} \Omega \int_{z_{-}(x)}^{z_{+}(x)} \mathrm{d} \tilde{z}_{i}\left[z_{+}(x)-\tilde{z}_{i}\right]^{-\epsilon}\left[\tilde{z}_{i}-z_{-}(x)\right]^{-\epsilon}
\end{aligned}
$$

with the limits

$$
z_{ \pm}(x)=\frac{1-x+\mu_{i j}^{2}+\mu_{i}^{2}-\mu_{j}^{2} \pm \sqrt{\left(1-x+\mu_{i j}^{2}-\mu_{i}^{2}-\mu_{j}^{2}\right)^{2}-4 \mu_{i}^{2} \mu_{j}^{2}}}{2\left(1-x+\mu_{i j}^{2}\right)} .
$$

The $\mathrm{d}^{d-3} \Omega$ integration is over the solid angle $\Omega$ perpendicular to $\widetilde{p}_{i j}$ and $p_{a}$.

\subsubsection{The dipole splitting functions}

We give the functions $\mathbf{V}_{i j}^{a}$ in Eq. (5.40) for the two different QCD splitting processes $\tilde{i j} \rightarrow i+j$ :

- $Q \rightarrow g\left(p_{i}\right)+Q\left(p_{j}\right): \quad m_{i}=0$ and $m_{j}=m_{i j}=m_{Q}$,

- $g \rightarrow Q\left(p_{i}\right)+\bar{Q}\left(p_{j}\right): \quad m_{i}=m_{j}=m_{Q}$ and $m_{i j}=0$.

The case $\bar{Q} \rightarrow g \bar{Q}$ is formally identical to $Q \rightarrow g Q$. The splitting $g \rightarrow g g$ is already given in Eq. (5.40) of Ref. [11], since no massive parton is involved in this case. Specifically, we define the dipole functions

$$
\begin{aligned}
\left\langle s\left|\mathbf{V}_{g Q}^{a}\right| s^{\prime}\right\rangle & =8 \pi \mu^{2 \epsilon} \alpha_{\mathrm{s}} C_{\mathrm{F}}\left\{\frac{2}{2-x_{i j, a}-\tilde{z}_{j}}-1-\tilde{z}_{j}-\frac{m_{Q}^{2}}{p_{i} p_{j}}-\epsilon\left(1-\tilde{z}_{j}\right)\right\} \delta_{s s^{\prime}} \\
& =\left\langle\mathbf{V}_{g Q}^{a}\right\rangle \delta_{s s^{\prime}}, \\
\left\langle\mu\left|\mathbf{V}_{Q \bar{Q}}^{a}\right| \nu\right\rangle & =8 \pi \mu^{2 \epsilon} \alpha_{\mathrm{s}} T_{\mathrm{R}}\left\{-g^{\mu \nu}-\frac{4}{\left(p_{i}+p_{j}\right)^{2}}\left[\tilde{z}_{i} p_{i}^{\mu}-\tilde{z}_{j} p_{j}^{\mu}\right]\left[\tilde{z}_{i} p_{i}^{\nu}-\tilde{z}_{j} p_{j}^{\nu}\right]\right\}, \\
\left\langle\mathbf{V}_{Q \bar{Q}}^{a}\right\rangle & =8 \pi \mu^{2 \epsilon} \alpha_{\mathrm{s}} T_{\mathrm{R}}\left\{1-\frac{2}{1-\epsilon}\left(z_{+}-\tilde{z}_{i}\right)\left(\tilde{z}_{i}-z_{-}\right)\right\},
\end{aligned}
$$

with $z_{ \pm}$given in Eq. (5.49).

In App. Q, we give the dipole splitting functions $\mathbf{V}_{i j}^{a}$ that are relevant for SUSY QCD calculations. 


\subsubsection{The integrated dipole functions}

The integral of the spin-averaged dipole functions $\left\langle\mathbf{V}_{i j}^{a}\right\rangle$ over the singular phase space $\left[\mathrm{d} p_{i}\left(\widetilde{p}_{i j} ; p_{a}, x\right)\right]$ is defined by

$$
\int\left[\mathrm{d} p_{i}\left(\widetilde{p}_{i j} ; p_{a}, x\right)\right] \frac{1}{\left(p_{i}+p_{j}\right)^{2}-m_{i j}^{2}}\left\langle\mathbf{V}_{i j}^{a}\right\rangle \equiv \frac{\alpha_{\mathrm{s}}}{2 \pi} \frac{1}{\Gamma(1-\epsilon)}\left(\frac{4 \pi \mu^{2}}{2 \widetilde{p}_{i j} p_{a}}\right)^{\epsilon} I_{i j}^{a}(x ; \epsilon),
$$

where $I_{i j}^{a}(x ; \epsilon)$ depends also on $\widetilde{p}_{i j} \cdot p_{a}$ and the parton masses. In the massless case, $I_{i j}^{a}$ does not depend on $\widetilde{p}_{i j} \cdot p_{a}$ and becomes the function $\mathcal{V}_{i j}(x ; \epsilon)$ of Ref. [11.

For $\epsilon=0$ the function $I_{g Q}^{a}(x ; \epsilon)$ is well-defined everywhere, except at the endpoint $x=1$ where the integral is singular as $\frac{\ln (1-x)}{1-x}$. To avoid this singularity, the limit $\epsilon \rightarrow 0$ has to be performed uniformly in $x$, which is achieved by decomposing $I_{g Q}^{a}(x ; \epsilon)$ into a continuum and an endpoint part using the usual ' + '-distribution, which is defined by

$$
\int_{0}^{1} \mathrm{~d} x(f(x))_{+} g(x) \equiv \int_{0}^{1} \mathrm{~d} x f(x)[g(x)-g(1)] .
$$

In this manner, $I_{g Q}^{a}(x ; \epsilon)$ defines an $x$-distribution whose coefficients contain poles in $\epsilon$.

In the case of the splitting process $g \rightarrow Q \bar{Q}$, the endpoint is located at $x_{+}=1-4 \mu_{Q}^{2}$, where the function $I_{Q \bar{Q}}^{a}(x ; \epsilon)$ is not singular. However, it becomes singular as $\frac{1}{1-x}$ for vanishing quark masses. To ensure a smooth massless behaviour, the limit $\epsilon \rightarrow 0$ has to be performed uniformly both in $x$ and in the quark mass. To achieve this, we have to use a modified version of the '+'-distribution, which we call ' $x_{+}$'-distribution,

$$
\int_{0}^{1} \mathrm{~d} x(f(x))_{x_{+}} g(x) \equiv \int_{0}^{1} \mathrm{~d} x f(x) \Theta\left(x_{+}-x\right)\left[g(x)-g\left(x_{+}\right)\right] .
$$

The ' $x_{+}$'-distribution reduces to the usual ' + '-distribution by setting $x_{+}=1$ and, in particular, it tends to the '+'-distribution smoothly when $\mu_{Q} \rightarrow 0$ in $I_{Q \bar{Q}}^{a}(x ; \epsilon)$.

After decomposing $I_{i j}^{a}$ into a continuum and an endpoint part, we further decompose the endpoint part into a singular $\left(J_{i j}^{a ; \mathrm{S}}\right)$ and a finite $\left(J_{i j}^{a ; \mathrm{NS}}\right)$ piece, such that the latter vanishes for vanishing masses:

$$
\begin{aligned}
I_{g Q}^{a}(x ; \epsilon) & =C_{\mathrm{F}}\left\{\left[J_{g Q}^{a}\left(x, \mu_{Q}\right)\right]_{+}+\delta(1-x)\left[J_{g Q}^{a ; \mathrm{S}}\left(\mu_{Q} ; \epsilon\right)+J_{g Q}^{a ; \mathrm{NS}}\left(\mu_{Q}\right)\right]\right\}+\mathrm{O}(\epsilon), \\
I_{Q \bar{Q}}^{a}(x ; \epsilon) & =T_{\mathrm{R}}\left\{\left[J_{Q \bar{Q}}^{a}\left(x, \mu_{Q}\right)\right]_{x_{+}}+\delta\left(x_{+}-x\right)\left[J_{Q \bar{Q}}^{a ; \mathrm{S}}\left(\mu_{Q} ; \epsilon\right)+J_{Q \bar{Q}}^{a ; \mathrm{NS}}\left(\mu_{Q}\right)\right]\right\}+\mathrm{O}(\epsilon),
\end{aligned}
$$

where $x_{+}=1-4 \mu_{Q}^{2}$ and $J_{i j}^{a ; \mathrm{NS}}\left(\mu_{Q}=0\right)=0$.

The three contributions to Eq. (5.56) are

$$
\begin{gathered}
{\left[J_{g Q}^{a}\left(x, \mu_{Q}\right)\right]_{+}=} \\
\left(\frac{1-x}{2\left(1-x+\mu_{Q}^{2}\right)^{2}}-\frac{2}{1-x}\left[1+\ln \left(1-x+\mu_{Q}^{2}\right)\right]\right)_{+} \\
+\left(\frac{2}{1-x}\right)_{+} \ln \left(2+\mu_{Q}^{2}-x\right) \\
J_{g Q}^{a ; S}\left(\mu_{Q} ; \epsilon\right)=\frac{1}{\epsilon^{2}}-\mu_{Q}^{-2 \epsilon}\left(\frac{1}{\epsilon^{2}}+\frac{1}{2 \epsilon}+\frac{\pi^{2}}{6}+2\right)-\frac{1}{\epsilon} \ln \left(1+\mu_{Q}^{2}\right)+\frac{3}{2 \epsilon}+\frac{7}{2}-\frac{\pi^{2}}{2},
\end{gathered}
$$




$$
\begin{aligned}
J_{g Q}^{a ; \mathrm{NS}}\left(\mu_{Q}\right) & =\frac{1}{2} \ln ^{2}\left(1+\mu_{Q}^{2}\right)-2 \ln \mu_{Q}^{2} \ln \left(1+\mu_{Q}^{2}\right)-4 \mathrm{Li}_{2}\left(-\mu_{Q}^{2}\right) \\
& +\frac{1}{2} \ln \left(1+\mu_{Q}^{2}\right)+\frac{\mu_{Q}^{2}}{2\left(1+\mu_{Q}^{2}\right)} .
\end{aligned}
$$

Note that the notation in Eq. (5.58) is symbolic: $\left[J_{g Q}^{a}\left(x, \mu_{Q}\right)\right]_{+}$contains all the plus distribution contributions, but is not itself a plus distribution. The same is true of $\left[J_{g g}^{a}(x)\right]_{+}$ defined in Eq. (5.67).

Setting the mass of the quark to zero, we immediately obtain the corresponding expression in the massless theory (see Eq. (5.57) in Ref. [11]). If the quark mass is non-vanishing, we can expand the singular part of the endpoint contribution as

$$
J_{g Q}^{a ; \mathrm{S}}\left(\mu_{Q}>0\right)=\frac{1}{\epsilon} \ln \frac{\mu_{Q}^{2}}{1+\mu_{Q}^{2}}-\frac{1}{2} \ln ^{2} \mu_{Q}^{2}+\frac{1}{\epsilon}+\frac{1}{2} \ln \mu_{Q}^{2}+\frac{3}{2}-\frac{2}{3} \pi^{2} .
$$

The three contributions to Eq. (5.57) are

$$
\begin{gathered}
{\left[J_{Q \bar{Q}}^{a}\left(x, \mu_{Q}\right)\right]_{x_{+}}=\frac{2}{3}\left(\frac{1-x+2 \mu_{Q}^{2}}{(1-x)^{2}} \sqrt{1-\frac{4 \mu_{Q}^{2}}{1-x}}\right)_{x_{+}},} \\
J_{Q \bar{Q}}^{a ; S}\left(\mu_{Q} ; \epsilon\right)=-\frac{2}{3 \epsilon}\left(1-\mu_{Q}^{-2 \epsilon}\right)-\frac{10}{9}, \\
J_{Q \bar{Q}}^{a ; \mathrm{NS}}\left(\mu_{Q}\right)=\frac{10}{9}\left(1-\sqrt{1-4 \mu_{Q}^{2}}\right)-\frac{8}{9} \mu_{Q}^{2} \sqrt{1-4 \mu_{Q}^{2}}+\frac{4}{3} \ln \left(\frac{1+\sqrt{1-4 \mu_{Q}^{2}}}{2}\right) .
\end{gathered}
$$

The massless result of Eq. (5.58) in Ref. [1] is again recovered by simply setting $m_{Q}=0$. If $m_{Q} \neq 0$, the collinear pole $1 / \epsilon$ is replaced by the logarithm of the scaled quark mass,

$$
J_{Q \bar{Q}}^{a ; \mathrm{S}}\left(\mu_{Q}>0\right)=-\frac{2}{3} \ln \mu_{Q}^{2}-\frac{10}{9} .
$$

As mentioned above, the case of the splitting process $g \rightarrow g g$ is already discussed in Ref. [1]. We recall the result by using the present notation:

$$
I_{g g}^{a}(x ; \epsilon)=2 C_{\mathrm{A}}\left\{\left[J_{g g}^{a}(x)\right]_{+}+\delta(1-x) J_{g g}^{a ; \mathrm{S}}(\epsilon)\right\}+\mathrm{O}(\epsilon)
$$

where

$$
\begin{aligned}
{\left[J_{g g}^{a}(x)\right]_{+} } & =\left(\frac{2}{1-x} \ln \frac{1}{1-x}-\frac{11}{6} \frac{1}{1-x}\right)_{+}+\frac{2}{1-x} \ln (2-x) \\
J_{g g}^{a ; \mathrm{S}}(x) & =\frac{1}{\epsilon^{2}}+\frac{11}{6 \epsilon}+\frac{67}{18}-\frac{\pi^{2}}{2} .
\end{aligned}
$$

Finally, we consider the asymptotics in the case of a parametrically-small quark mass $m_{Q}=\mu_{Q} \sqrt{2 \widetilde{p}_{i j} p_{a}}$, used as collinear regulator:

$$
\begin{aligned}
I_{g Q}^{a}\left(x, \mu_{Q}\right) \underset{\mu_{Q} \rightarrow 0}{\simeq} C_{\mathrm{F}} & {\left[-2\left(\frac{\ln (1-x)}{1-x}\right)_{+}-\frac{3}{2}\left(\frac{1}{1-x}\right)_{+}+\frac{2}{1-x} \ln (2-x)\right.} \\
+ & \left.\delta(1-x)\left(\frac{1}{\epsilon}\left(\ln \mu_{Q}^{2}+1\right)-\frac{1}{2} \ln ^{2} \mu_{Q}^{2}+\frac{1}{2} \ln \mu_{Q}^{2}-\frac{2}{3} \pi^{2}+\frac{3}{2}\right)\right],
\end{aligned}
$$




$$
I_{Q \bar{Q}}^{a}\left(x, \mu_{Q}\right) \underset{\mu_{Q} \rightarrow 0}{\sim} T_{\mathrm{R}}\left[\frac{2}{3}\left(\frac{1}{1-x}\right)_{+}-\delta(1-x)\left(\frac{2}{3} \ln \mu_{Q}^{2}+\frac{10}{9}\right)\right] .
$$

Analogously to Eq. (5.39), on the right-hand side we have neglected all the contributions of $\mathrm{O}\left(\mu_{Q}\right)$, independently of their $\epsilon$ dependence. Comparing these results with Eqs. (5.57) and (5.58) in Ref. [11], we notice that the continuum parts reduce to those of the exactly massless case, as expected since all the singularities are related to the final-state emitter. The $1 / \epsilon$ collinear poles of the massless theory in the endpoint parts are replaced by the mass logarithms, which in the $Q \rightarrow g Q$ splitting are related to those of the fermion-photon splitting [25].

We conclude this section with a comment on a subtle point related to the definition of the $x$-distributions or, more precisely, to the definition of the space of test functions onto which the $x$-distributions act. Indeed, the integrals $I_{i j}^{a}$ depend on two variables, $x$ and $\widetilde{p}_{i j}$, which are both integration variables in the calculation of the cross section. Our definition in Eqs. (5.56) and (5.57), in terms of continuum and endpoint parts, considers $I_{i j}^{a}$ as an $x$-distribution that acts on functions of the variables $x$ and $\widetilde{p}_{i j}$, and $\widetilde{p}_{i j}$ is regarded as an additional and independent integration variable that has to be kept fixed in the subtraction prescriptions of Eqs. (5.54) and (5.55). However, the $x$-distributions can also be defined in a different way, for instance, by regarding $Q=\widetilde{p}_{i j}-x p_{a}$ (instead of $\widetilde{p}_{i j}$ ) as the independent integration variable. This alternative definition is explicitly worked out in App. B, where we show that the replacement $\widetilde{p}_{i j} \rightarrow Q=\widetilde{p}_{i j}-x p_{a}$, being $x$ dependent, has to be handled with care in the definition of the $x$-distributions.

\subsection{Initial-state emitter and final-state spectator}

We define the dipole contribution $\mathcal{D}_{j}^{a i}$ (see Fig. 1) to the factorization formula (5.1) in close analogy to that in Eq. (5.2):

$$
\begin{aligned}
& \mathcal{D}_{j}^{a i}\left(p_{1}, \ldots, p_{m+1} ; p_{a}, \ldots\right)= \\
& \left.\quad-\frac{1}{2 p_{a} p_{i}} \frac{1}{x_{i j, a}} \underset{m, \widetilde{a i}}{\langle\ldots, \tilde{j}}, \ldots ; \widetilde{a i}, \ldots\left|\frac{\boldsymbol{T}_{j} \cdot \boldsymbol{T}_{a i}}{\boldsymbol{T}_{a i}^{2}} \mathbf{V}_{j}^{a i}\right| \ldots, \tilde{j}, \ldots ; \widetilde{a i}, \ldots\right\rangle_{m, \widetilde{a i}}
\end{aligned}
$$

The $m$-parton matrix elements on the right-hand side are obtained from the original $(m+1)$ parton matrix elements by replacing the initial-state parton $a$ with the emitter parton $\widetilde{a i}$, the final-state parton $j$ with $\tilde{j}$ and dropping the final-state parton $i$.

The final-state parton momentum $p_{i}$ and the initial-state momentum $p_{a}$ are massless and the mass of spectator momentum $p_{j}$ is arbitrary. The total transferred momentum is denoted by $Q$ (not to be confused with $Q$ in Eq. (5.3)),

$$
p_{i}^{2}=0, \quad p_{j}^{2}=m_{j}^{2}, \quad p_{a}^{2}=0, \quad Q=p_{i}+p_{j}-p_{a} .
$$

The on-shell mass of the emitter is $m_{a i}=0$. 


\subsubsection{Kinematics and phase-space factorization}

We can completely take over the kinematics of Sect. 5.2.1 after replacing $\tilde{p}_{a} \rightarrow \tilde{p}_{a i}, \tilde{p}_{i j} \rightarrow \tilde{p}_{j}$, $m_{i} \rightarrow 0$ and $m_{i j} \rightarrow m_{j}$ there. Thus, the new momenta read

$$
\tilde{p}_{a i}^{\mu}=x_{i j, a} p_{a}^{\mu}, \quad \tilde{p}_{j}^{\mu}=p_{i}^{\mu}+p_{j}^{\mu}-\left(1-x_{i j, a}\right) p_{a}^{\mu},
$$

where

$$
x_{i j, a}=\frac{p_{a} p_{i}+p_{a} p_{j}-p_{i} p_{j}}{p_{a} p_{i}+p_{a} p_{j}}, \quad \tilde{z}_{i}=\frac{p_{a} p_{i}}{p_{a} p_{i}+p_{a} p_{j}}, \quad \tilde{z}_{j}=\frac{p_{a} p_{j}}{p_{a} p_{i}+p_{a} p_{j}} .
$$

They obey the mass-shell relations

$$
p_{a}^{2}=p_{i}^{2}=\tilde{p}_{a i}^{2}=0, \quad p_{j}^{2}=\tilde{p}_{j}^{2}=m_{j}^{2}
$$

and momentum conservation

$$
Q=p_{i}+p_{j}-p_{a}=\tilde{p}_{j}-\tilde{p}_{a i}
$$

The rescaled mass $\mu_{j}$ of the parton $j$ is defined by

$$
\mu_{j}=\frac{m_{j}}{\sqrt{2 \tilde{p}_{j} p_{a}}}
$$

For the sake of completeness we also write down the phase-space factorization,

$$
\int \mathrm{d} \phi\left(p_{i}, p_{j} ; Q+p_{a}\right)=\int_{0}^{1} \mathrm{~d} x \int \mathrm{d} \phi\left(\tilde{p}_{j} ; Q+x p_{a}\right) \int\left[\mathrm{d} p_{i}\left(\tilde{p}_{j} ; p_{a}, x\right)\right]
$$

with

$$
\begin{aligned}
\int\left[\mathrm{d} p_{i}\left(\tilde{p}_{j} ; p_{a}, x\right)\right]= & \frac{1}{4}(2 \pi)^{-3+2 \epsilon}\left(2 \tilde{p}_{j} p_{a}\right)^{1-\epsilon} \int_{0}^{1} \mathrm{~d} x_{i j, a} \delta\left(x-x_{i j, a}\right)\left(1-x+\mu_{j}^{2}\right)^{-\epsilon} \\
& \times \int \mathrm{d}^{d-3} \Omega \int_{0}^{z_{+}(x)} \mathrm{d} \tilde{z}_{i}\left[z_{+}(x)-\tilde{z}_{i}\right]^{-\epsilon} \tilde{z}_{i}^{-\epsilon}
\end{aligned}
$$

where the upper limit of the $z$-integration is

$$
z_{+}(x)=\frac{1-x}{1-x+\mu_{j}^{2}}
$$

\subsubsection{The dipole functions}

We consider the splittings $q \rightarrow q g$ or $g q, g \rightarrow q \bar{q}$ or $\bar{q} q, g \rightarrow g g$ of massless partons in the presence of the massive spectator parton $j$. The corresponding dipole functions read as follows:

$$
\begin{aligned}
\left\langle s\left|\mathbf{V}_{j}^{q g}\right| s^{\prime}\right\rangle & =8 \pi \mu^{2 \epsilon} \alpha_{\mathrm{s}} C_{\mathrm{F}}\left\{\frac{2}{2-x_{i j, a}-\tilde{z}_{j}}-1-x_{i j, a}-\epsilon\left(1-x_{i j, a}\right)\right\} \delta_{s s^{\prime}} \\
& =\left\langle\mathbf{V}_{j}^{q g}\right\rangle \delta_{s s^{\prime}}
\end{aligned}
$$




$$
\begin{aligned}
\left\langle s\left|\mathbf{V}_{j}^{g \bar{q}}\right| s^{\prime}\right\rangle= & 8 \pi \mu^{2 \epsilon} \alpha_{\mathrm{s}} T_{\mathrm{R}}\left\{1-\epsilon-2 x_{i j, a}\left(1-x_{i j, a}\right)\right\} \delta_{s s^{\prime}} \\
= & \left\langle\mathbf{V}_{j}^{g \bar{q}}\right\rangle \delta_{s s^{\prime}} \\
\left\langle\mu\left|\mathbf{V}_{j}^{q q}\right| \nu\right\rangle= & 8 \pi \mu^{2 \epsilon} \alpha_{\mathrm{s}} C_{\mathrm{F}}\left\{-g^{\mu \nu} x_{i j, a}+\frac{1-x_{i j, a}}{x_{i j, a}} \frac{2 \tilde{z}_{i} \tilde{z}_{j}}{p_{i} p_{j}}\left(\frac{p_{i}^{\mu}}{\tilde{z}_{i}}-\frac{p_{j}^{\mu}}{\tilde{z}_{j}}\right)\left(\frac{p_{i}^{\nu}}{\tilde{z}_{i}}-\frac{p_{j}^{\nu}}{\tilde{z}_{j}}\right)\right\} \\
\left\langle\mathbf{V}_{j}^{q q}\right\rangle= & 8 \pi \mu^{2 \epsilon} \alpha_{\mathrm{s}} C_{\mathrm{F}}\left\{x_{i j, a}+\frac{2\left(1-x_{i j, a}\right)}{x_{i j, a}(1-\epsilon)}-\frac{2 \mu_{j}^{2}}{x_{i j, a}(1-\epsilon)} \frac{\tilde{z}_{i}}{\tilde{z}_{j}}\right\} \\
\left\langle\mu\left|\mathbf{V}_{j}^{g g}\right| \nu\right\rangle=16 \pi \mu^{2 \epsilon} \alpha_{\mathrm{s}} C_{\mathrm{A}} & \left\{-g^{\mu \nu}\left[\frac{1}{2-x_{i j, a}-\tilde{z}_{j}}-1+x_{i j, a}\left(1-x_{i j, a}\right)\right]\right. \\
\left\langle\mathbf{V}_{j}^{g g}\right\rangle=16 \pi \mu^{2 \epsilon} \alpha_{\mathrm{s}} C_{\mathrm{A}} & \left\{\frac{1-x_{i j, a}}{\tilde{z}_{i} \tilde{z}_{j}} \tilde{p}_{i}\left(\frac{p_{i}^{\mu}}{\tilde{z}_{i}}-\frac{p_{j}^{\mu}}{\tilde{z}_{j}}\right)\left(\frac{p_{i}^{\nu}}{\tilde{z}_{i}}-\frac{p_{j}^{\nu}}{\tilde{z}_{j}}\right)\right\}
\end{aligned}
$$

\subsubsection{The integrated dipole functions}

We define the integral of $\left\langle\mathbf{V}_{j}^{a i}\right\rangle$ over the singular phase space $\left[\mathrm{d} p_{i}\left(\tilde{p}_{j} ; p_{a}, x\right)\right]$ by

$$
\int\left[\mathrm{d} p_{i}\left(\tilde{p}_{j} ; p_{a}, x\right)\right] \frac{1}{2 p_{a} p_{i}} \frac{n_{\mathrm{s}}(\widetilde{a i})}{n_{\mathrm{s}}(a)}\left\langle\mathbf{V}_{j}^{a i}\right\rangle \equiv \frac{\alpha_{\mathrm{s}}}{2 \pi} \frac{1}{\Gamma(1-\epsilon)}\left(\frac{4 \pi \mu^{2}}{2 \tilde{p}_{j} p_{a}}\right)^{\epsilon} I_{j}^{a, \widetilde{a i}}\left(x, \mu_{j} ; \epsilon\right),
$$

where the factor $n_{\mathrm{s}}(\widetilde{a i}) / n_{\mathrm{s}}(a)$ turns the spin-average over $\widetilde{a i}$ into the one over $a$. Splitting off the massless part $I_{j}^{a b}(x, 0 ; \epsilon)$, the results for $I_{j}^{a b}\left(x, \mu_{j} ; \epsilon\right)$ can be written in the compact form

$$
\begin{aligned}
I_{j}^{a b}\left(x, \mu_{j} ; \epsilon\right) & =I_{j}^{a b}(x, 0 ; \epsilon)+P_{\text {reg }}^{a b}(x) \ln \left(\frac{1-x}{1-x+\mu_{j}^{2}}\right) \\
& +\delta_{g b} \boldsymbol{T}_{a}^{2} \frac{2 \mu_{j}^{2}}{x} \ln \left(\frac{\mu_{j}^{2}}{1-x+\mu_{j}^{2}}\right)+2 \delta^{a b} \boldsymbol{T}_{a}^{2}\left(\frac{1}{1-x}\right)_{+} \ln \left(\frac{2-x}{2-x+\mu_{j}^{2}}\right) \\
& +\delta^{a b} \delta(1-x) \boldsymbol{T}_{a}^{2}\left[\frac{1}{\epsilon} \ln \left(1+\mu_{j}^{2}\right)+\frac{1}{2} \ln ^{2}\left(1+\mu_{j}^{2}\right)+2 \operatorname{Li}_{2}\left(\frac{1}{1+\mu_{j}^{2}}\right)-\frac{\pi^{2}}{3}\right] \\
& +\mathrm{O}(\epsilon),
\end{aligned}
$$

where the auxiliary functions $P_{\text {reg }}^{a b}(x)$ are given by

$$
\begin{array}{ll}
P_{\text {reg }}^{q q}(x)=-C_{\mathrm{F}}(1+x), & P_{\text {reg }}^{g q}(x)=T_{\mathrm{R}}\left[x^{2}+(1-x)^{2}\right], \\
P_{\text {reg }}^{q g}(x)=C_{\mathrm{F}} \frac{1+(1-x)^{2}}{x}, & P_{\text {reg }}^{g g}(x)=2 C_{\mathrm{A}}\left[\frac{1-x}{x}-1+x(1-x)\right] .
\end{array}
$$

Since the kinematics of the spectator parton $j$ is not connected with any singularity, the limit $m_{j} \rightarrow 0$ is smooth in all cases. The massless part can be written in the following 
compact form,

$$
\begin{aligned}
I_{j}^{a b}(x, 0 ; \epsilon) & =-\frac{1}{\epsilon} P^{a b}(x)+P_{\mathrm{reg}}^{a b}(x) \ln (1-x)+2 \delta^{a b} \boldsymbol{T}_{a}^{2}\left[2\left(\frac{\ln (1-x)}{1-x}\right)_{+}-\frac{\ln (2-x)}{1-x}\right] \\
& +\hat{P}^{\prime a b}(x)+\delta^{a b} \delta(1-x)\left[\boldsymbol{T}_{a}^{2}\left(\frac{1}{\epsilon^{2}}+\frac{\pi^{2}}{6}\right)+\gamma_{a} \frac{1}{\epsilon}\right]+\mathrm{O}(\epsilon),
\end{aligned}
$$

where $\gamma_{a}$ are the following flavour constants:

$$
\gamma_{q}=\frac{3}{2} C_{\mathrm{F}}, \quad \gamma_{g}=\frac{11}{6} C_{\mathrm{A}}-\frac{2}{3} T_{\mathrm{R}} N_{f}
$$

and $\hat{P}^{\prime a b}(x)$ is the $\epsilon$-dependent part of the $d$-dimensional Altarelli-Parisi splitting functions in Eqs. 4.13 4.16):

$$
\left\langle\hat{P}_{a b}(x ; \epsilon)\right\rangle=\left\langle\hat{P}_{a b}(x ; \epsilon=0)\right\rangle-\epsilon \hat{P}^{\prime a b}(x)+\mathrm{O}\left(\epsilon^{2}\right) .
$$

Explicitly,

$$
\hat{P}^{\prime q q}(x)=\hat{P}^{\prime q g}(1-x)=C_{\mathrm{F}}(1-x), \quad \hat{P}^{\prime g q}(x)=2 T_{\mathrm{R}} x(1-x), \quad \hat{P}^{\prime g g}(x)=0 .
$$

The functions $P^{a b}(x)$ in Eq. (5.90) are the usual regularized Altarelli-Parisi functions that can be written as 11

$$
P^{a b}(x)=P_{\mathrm{reg}}^{a b}(x)+\delta^{a b}\left[2 \boldsymbol{T}_{a}^{2}\left(\frac{1}{1-x}\right)_{+}+\gamma_{a} \delta(1-x)\right] .
$$

\subsection{Initial-state emitter and initial-state spectator}

Since we allow for massive partons only in the final state in this paper, the case of emitter and spectator both from the initial state is already completely covered in Ref. [11], where all cases for massless QCD partons are presented. Results for the $Q \rightarrow Q g$ splitting with

a massive quark in the initial state can be deduced from the treatment of photon emission off fermions described in Ref. [25].

\section{QCD cross sections at NLO}

In this section we describe our method for evaluating QCD cross sections of massive and/or massless partons. We start with the precise definitions of the three terms on the right-hand side of Eq. (2.13), which can be considered the final expressions, ready for implementing in a partonic Monte Carlo program. Then we derive the explicit forms of the insertion operators $\boldsymbol{I}, \boldsymbol{P}$ and $\boldsymbol{K}$ relevant to the three different cases of initial state: elementary particle reactions involving zero, one or two initial-state hadrons. More details on our notation can be found in Sects. 6-11 of Ref. [11. 


\subsection{Final formulae}

In order to present the final formulae of our algorithm, we start with the most general case of two incident hadrons and then point out the simplifications if one or both of the incoming particles are leptons.

In the case of processes with two initial-state hadrons carrying momenta $p_{A}^{\mu}$ and $p_{B}^{\mu}$, the calculation of the QCD cross section requires the introduction of parton distributions. If we denote by $f_{a / A}\left(\eta, \mu_{F}^{2}\right)$ the density of partons of type $a$ in the incoming hadron $A$, the hadronic cross section is given by

$$
\sigma\left(p_{A}, p_{B}\right)=\sum_{a, b} \int_{0}^{1} \mathrm{~d} \eta_{a} f_{a / A}\left(\eta_{a}, \mu_{F}^{2}\right) \int_{0}^{1} \mathrm{~d} \eta_{b} f_{b / B}\left(\eta_{b}, \mu_{F}^{2}\right) \sigma_{a b}\left(\eta_{a} p_{A}, \eta_{b} p_{B}\right),
$$

where $\sigma_{a b}$ is the partonic cross section, which in the first two orders of the perturbative expansion has the schematic form given in Eq. (2.1),

$$
\sigma_{a b}\left(p_{a}, p_{b}\right)=\sigma_{a b}^{\mathrm{LO}}\left(p_{a}, p_{b}\right)+\sigma_{a b}^{\mathrm{NLO}}\left(p_{a}, p_{b} ; \mu_{F}^{2}\right)
$$

If one or two of the colliding particles is pointlike, i.e. a lepton in the Standard Model, then the integration becomes trivial by replacing the corresponding parton density by $\delta(1-\eta) \delta_{a A}$. The LO parton-level cross section is given by

$$
\begin{aligned}
\sigma_{a b}^{\mathrm{LO}}\left(p_{a}, p_{b}\right) & =\int_{m} \mathrm{~d} \sigma_{a b}^{\mathrm{B}}\left(p_{a}, p_{b}\right) \\
& =\int \mathrm{d} \Phi^{(m)}\left(p_{a}, p_{b}\right) \frac{1}{n_{c}(a) n_{c}(b)}\left|\mathcal{M}_{m, a b}\left(\left\{p_{i}, m_{i}\right\} ; p_{a}, p_{b}\right)\right|^{2} F_{J}^{(m)}\left(\left\{p_{i}\right\} ; p_{a}, p_{b}\right)
\end{aligned}
$$

where $a$ and $b$ denote the flavours of the incoming partons, $p_{a}$ and $p_{b}$ are their momenta and $n_{c}(a), n_{c}(b)$ are their number of colours (for an incoming lepton $n_{c}$ is set to 1 ). The matrix element $\left|\mathcal{M}_{m, a b}\right|^{2}$ is the square of the tree-level amplitude to produce $m$ final-state partons. The factor

$$
\mathrm{d} \Phi^{(m)}\left(p_{a}, p_{b}\right)=\mathcal{N}_{i n} \frac{1}{n_{s}(a) n_{s}(b) \mathcal{F}\left(p_{a} \cdot p_{b}\right)} \sum_{\{m\}} \mathrm{d} \phi_{m}\left(p_{1}, \ldots, p_{m} ; p_{a}+p_{b}\right) \frac{1}{S_{\{m\}}}
$$

contains (i) the averaging over the number of initial-state polarizations $\left(n_{s}(i)\right.$ number of states for parton $i, i=a$ or $b$ ), (ii) the flux factor $\mathcal{F}\left(p_{a} \cdot p_{b}\right)$, (iii) a summation over all configurations with $m$ partons in the final state, (iv) the Lorentz invariant phase space of $m$ final-state partons $\mathrm{d} \phi_{m},(\mathrm{v})$ a Bose symmetry factor $S_{\{m\}}$ for identical partons in the final state and (vi) $\mathcal{N}_{i n}$, which includes all factors that are QCD independent. Finally, the function $F_{J}^{(m)}$ in Eq. (6.3) defines the jet observable we want to compute. It has to fulfil the formal requirements of infrared and collinear safety. Furthermore, in order for our cross section to have a smooth small-mass limit, $F_{J}$ should also be quasi-collinear safe.

The general definition of quasi-collinear safety is analogous to that of collinear safety: if a pair of partons $i$ and $j$ whose masses and relative transverse momenta are all $\mathrm{O}(\lambda Q)$, where $Q$ is the hard scale, then as $\lambda \rightarrow 0, F_{J}^{(m+1)}\left(\ldots, p_{i}, p_{j}, \ldots\right)$ must tend to the value of $F_{J}^{(m)}\left(\ldots, p_{i}+p_{j}, \ldots\right)$. Any collinear-safe observable for massless partons can be extended 
to be quasi-collinear-safe for massive partons. However, there is a subtlety related to the flavour-dependence of $F_{J}$. To respect infrared and collinear safety, an observable must not depend on the identities of massless partons in the final state. It can however depend on the identities of massive partons, for example the thrust distribution in $e^{+} e^{-}$annihilation events that contain at least one bottom quark is an infrared- and collinear-safe quantity. However it is not quasi-collinear safe, because events in which the $b \bar{b}$ pair is quasi-collinear contribute but identical events with the $b \bar{b}$ pair replaced by a gluon do not. Careful definition of the observable can render it quasi-collinear safe and hence finite in the high-energy limit. One solution in this example would be to only include events in which the $b$ and $\bar{b}$ lie in opposite thrust hemispheres. Our method is perfectly capable of calculating cross sections for observables that are not quasi-collinear safe, but our careful treatment of the quasi-collinear limit only guarantees improved numerical convergence for quasi-collinear safe observables.

According to the general notation in Eq. (2.13), the full NLO contribution is a sum of three terms,

$$
\begin{aligned}
\sigma_{a b}^{\mathrm{NLO}}\left(p_{a}, p_{b} ; \mu_{F}^{2}\right) & =\sigma_{a b}^{\mathrm{NLO}\{m+1\}}\left(p_{a}, p_{b}\right)+\sigma_{a b}^{\mathrm{NLO}\{m\}}\left(p_{a}, p_{b}\right) \\
& +\int_{0}^{1} \mathrm{~d} x\left[\hat{\sigma}_{a b}^{\mathrm{NLO}\{m\}}\left(x ; x p_{a}, p_{b}, \mu_{F}^{2}\right)+\hat{\sigma}_{a b}^{\mathrm{NLO}\{m\}}\left(x ; p_{a}, x p_{b}, \mu_{F}^{2}\right)\right] .
\end{aligned}
$$

The first contribution has $(m+1)$-parton kinematics and is given by the following expression

$$
\begin{aligned}
& \sigma_{a b}^{\mathrm{NLO}\{m+1\}}\left(p_{a}, p_{b}\right)=\int_{m+1} {\left[\left(\mathrm{~d} \sigma_{a b}^{\mathrm{R}}\left(p_{a}, p_{b}\right)\right)_{\epsilon=0}-\left(\sum_{\text {dipoles }} \mathrm{d} \sigma_{a b}^{\mathrm{B}}\left(p_{a}, p_{b}\right) \otimes \mathrm{d} V_{\text {dipole }}\right)_{\epsilon=0}\right] } \\
&=\int \mathrm{d} \Phi^{(m+1)}\left(p_{a}, p_{b}\right)\left[\frac{1}{n_{c}(a) n_{c}(b)}\left|\mathcal{M}_{m+1, a b}\left(\left\{p_{i}, m_{i}\right\} ; p_{a}, p_{b}\right)\right|^{2} F_{J}^{(m+1)}\left(\left\{p_{i}\right\} ; p_{a}, p_{b}\right)\right. \\
&\left.\quad-\sum_{\text {dipoles }}\left(\mathcal{D} \cdot F^{(m)}\right)\left(\left\{p_{i}, m_{i}\right\} ; p_{a}, p_{b}\right)\right]
\end{aligned}
$$

where $\mathcal{M}_{m+1, a b}$ is the tree-level matrix element with $m+1$ partons in the final state and $\sum_{\text {dipoles }}\left(\mathcal{D} \cdot F^{(m)}\right)\left(\left\{p_{i}, m_{i}\right\} ; p_{a}, p_{b}\right)$ is the following sum of the dipole functions:

$$
\begin{aligned}
& \sum_{\substack{\text { pairs } \\
i, j}} \sum_{k \neq i, j} \mathcal{D}_{i j, k}\left(p_{1}, \ldots, p_{m+1} ; p_{a}, p_{b}\right) F_{J}^{(m)}\left(p_{1}, \ldots \widetilde{p}_{i j}, \widetilde{p}_{k}, \ldots, p_{m+1} ; p_{a}, p_{b}\right) \\
& +\sum_{\substack{\text { pairs } \\
i, j}}\left[\mathcal{D}_{i j}^{a}\left(p_{1}, \ldots, p_{m+1} ; p_{a}, p_{b}\right) F_{J}^{(m)}\left(p_{1}, \ldots \widetilde{p}_{i j}, \ldots, p_{m+1} ; \widetilde{p}_{a}, p_{b}\right)+(a \leftrightarrow b)\right] \\
& +\sum_{i} \sum_{k \neq i}\left[\mathcal{D}_{k}^{a i}\left(p_{1}, \ldots, p_{m+1} ; p_{a}, p_{b}\right) F_{J}^{(m)}\left(p_{1}, \ldots \widetilde{p}_{k}, \ldots, p_{m+1} ; \widetilde{p}_{a i}, p_{b}\right)+(a \leftrightarrow b)\right] \\
& +\sum_{i}\left[\mathcal{D}^{a i, b}\left(p_{1}, \ldots, p_{m+1} ; p_{a}, p_{b}\right) F_{J}^{(m)}\left(\widetilde{p}_{1}, \ldots, \widetilde{p}_{m+1} ; \widetilde{p}_{a i}, p_{b}\right)+(a \leftrightarrow b)\right]
\end{aligned}
$$

where $(a \leftrightarrow b)$ indicates that the roles of $a$ and $b$ are reversed. For example, in the second line, $\mathcal{D}_{i j}^{a}$ becomes $\mathcal{D}_{i j}^{b}$ and $\widetilde{p}_{a}, p_{b}$ becomes $p_{a}, \widetilde{p}_{b}$. In the last line of Eq. (6.7), the function $F_{J}^{(m)}$ depends on the final-state momenta $\widetilde{p}_{1}, \ldots, \widetilde{p}_{m+1}$, which are obtained from the 
original momenta $p_{1}, \ldots, p_{m+1}$ by a Lorentz tranformation (see Sect. 5.5 and the Erratum in Ref. [11]).

If an incoming particle is a lepton, then the terms with $\mathcal{D}$ carrying the corresponding parton-flavour index are absent from the sum, i.e. for one initial-state hadron all terms corresponding to the $(a \leftrightarrow b)$ interchange and the full last line has to be dropped and for zero initial-state hadrons, only the first line remains.

The NLO contribution with $m$-parton kinematics is obtained by adding the virtual cross section and the singular part (with some accompanying finite terms) of the integral of the subtraction term over the factorized one-particle phase space. The former is given by the interference term between the tree and one-loop amplitudes $\left|\mathcal{M}_{m, a b}\right|_{(1-\text { loop })}^{2}$ and the latter in terms of an insertion operator $\boldsymbol{I}(\epsilon)$ :

$$
\begin{aligned}
& \sigma_{a b}^{\mathrm{NLO}\{m\}}\left(p_{a}, p_{b}\right)=\int_{m}\left[\mathrm{~d} \sigma_{a b}^{\mathrm{V}}\left(p_{a}, p_{b}\right)+\mathrm{d} \sigma_{a b}^{\mathrm{B}}\left(p_{a}, p_{b}\right) \otimes \boldsymbol{I}\right]_{\epsilon=0} \\
& =\int \mathrm{d} \Phi^{(m)}\left(p_{a}, p_{b}\right)\left[\frac{1}{n_{c}(a) n_{c}(b)}\left|\mathcal{M}_{m, a b}\left(\left\{p_{i}, m_{i}\right\} ; p_{a}, p_{b}\right)\right|_{(1-\mathrm{loop})}^{2}\right. \\
& \left.\quad+{ }_{m, a b}\langle 1, \ldots, m ; a, b|\boldsymbol{I}(\epsilon)| 1, \ldots, m ; a, b\rangle_{m, a b}\right]_{\epsilon=0} F_{J}^{(m)}\left(\left\{p_{i}\right\} ; p_{a}, p_{b}\right),
\end{aligned}
$$

where the operator $\boldsymbol{I}(\epsilon)$ is spelled out explicitly for the various cases of interest: in Eq. (6.16) to be inserted into ${ }_{m}\langle 1, \ldots, m \| 1, \ldots, m\rangle_{m}$ (case of no initial-state hadrons), (6.51) into $m, a\langle 1, \ldots, m ; a \| 1, \ldots, m ; a\rangle_{m, a}$ (case of one initial-state hadron) and (6.66) into ${ }_{m, a b}\langle 1, \ldots, m ; a, b \| 1, \ldots, m ; a, b\rangle_{m, a b}$ (case of two initial-state hadrons).

The third term on the right-hand side of Eq. (6.5) is a finite remainder left after cancellation of the $\epsilon$-poles of the collinear counterterm. It is a sum of as many contributions as the number of initial-state hadrons, each obtained by integrating a cross section with $m$-parton kinematics with respect to the fraction $x$ of the longitudinal momentum carried by one of the incoming partons. When this parton is the parton $a$, we explicitly have:

$$
\begin{array}{rl}
\int_{0}^{1} \mathrm{~d} & x \hat{\sigma}_{a b}^{\mathrm{NLO}\{m\}}\left(x ; x p_{a}, p_{b}, \mu_{F}^{2}\right)=\sum_{a^{\prime}} \int_{0}^{1} \mathrm{~d} x \int_{m}\left[\mathrm{~d} \sigma_{a^{\prime} b}^{\mathrm{B}}\left(x p_{a}, p_{b}\right) \otimes(\boldsymbol{K}+\boldsymbol{P})^{a, a^{\prime}}(x)\right]_{\epsilon=0} \\
= & \sum_{a^{\prime}} \int_{0}^{1} \mathrm{~d} x \int \mathrm{d} \Phi^{(m)}\left(x p_{a}, p_{b}\right) F_{J}^{(m)}\left(\left\{p_{i}\right\} ; x p_{a}, p_{b}\right) \\
& \times_{m, a^{\prime} b}\left\langle 1, \ldots, m ; x p_{a}, p_{b}\left|\boldsymbol{K}^{a, a^{\prime}}(x)+\boldsymbol{P}^{a, a^{\prime}}\left(x, \mu_{F}^{2}\right)\right| 1, \ldots, m ; x p_{a}, p_{b}\right\rangle_{m, a^{\prime} b},
\end{array}
$$

where the colour-charge operators $\boldsymbol{P}$ and $\boldsymbol{K}$ are respectively defined in Eqs. (6.53) and (6.55) to be inserted into $m, a^{\prime}\left\langle 1, \ldots, m ; x p_{a} \| 1, \ldots, m ; x p_{a}\right\rangle_{m, a^{\prime}}$ and Eqs. (6.67) and (6.68) into $_{m, a^{\prime} b}\left\langle 1, \ldots, m ; x p_{a}, p_{b} \| 1, \ldots, m ; x p_{a}, p_{b}\right\rangle_{m, a^{\prime} b}$. If there are two initial-state hadrons in the collision, then we also have to add the integral of $\hat{\sigma}_{a b}^{\mathrm{NLO}\{m\}}\left(x ; p_{a}, x p_{b}, \mu_{F}^{2}\right)$, which has a completely analogous expression to Eq. (6.9), apart from the replacements $x p_{a} \rightarrow p_{a}, p_{b} \rightarrow$ $x p_{b}$ and $\sum_{a^{\prime}} \rightarrow \sum_{b^{\prime}}$.

Equations (6.3), 6.6) and (6.9) are directly evaluated in four space-time dimensions. As for Eq. (6.8), one should first cancel analytically the $\epsilon$ poles of the one-loop matrix element with those of the insertion operator $\boldsymbol{I}$, perform the limit $\epsilon \rightarrow 0$ and then carry out the phase-space integration in four space-time dimensions. 
In the following subsections we derive the explicit form of the $\boldsymbol{I}, \boldsymbol{K}$ and $\boldsymbol{P}$ insertion operators, for the various specific cases, relevant to lepton-lepton, lepton-hadron and hadron-hadron scattering.

\subsection{Jet cross sections with no initial-state hadrons}

In processes with no initial-state hadrons, the QCD cross section for jet observables at NLO accuracy is given by Eqs. (2.1) and (2.2), and the NLO contribution is rewritten into the form of Eq. (2.3). In order to calculate the integral of the subtraction term over the factorized one-parton phase space, we start by writing $\int_{m+1} \mathrm{~d} \sigma^{\mathrm{A}}$ using the notation introduced in Eqs. (6.3), (6.4) and the phase-space factorization of Eq. (5.10). We find that the integral of the subtraction term can be written in the following form:

$$
\begin{array}{rl}
\int_{m+1} \mathrm{~d} \sigma^{\mathrm{A}}=\mathcal{N}_{i n} \sum_{\{m+1\}} \sum_{\substack{\text { pairs } \\
i, j}} \sum_{k \neq i, j} \int_{m} & \mathrm{~d} \phi_{m}\left(p_{1}, \ldots, \widetilde{p}_{i j}, \ldots, \widetilde{p}_{k}, \ldots, p_{m+1} ; Q\right) \\
& \times \frac{1}{S_{\{m+1\}}} F_{J}^{(m)}\left(p_{1}, \ldots, \widetilde{p}_{i j}, \ldots, \widetilde{p}_{k}, \ldots, p_{m+1}\right) \\
& \times \int\left[\mathrm{d} p_{i}\left(\widetilde{p}_{i j}, \widetilde{p}_{k}\right)\right] \mathcal{D}_{i j, k}\left(p_{1}, \ldots, p_{m+1}\right) .
\end{array}
$$

For the integration over $\mathrm{d} p_{i}$ we write the dipole factor as in Eq. (5.2),

$$
\mathcal{D}_{i j, k}\left(p_{1}, \ldots, p_{m+1}\right)=-\left[\frac{\boldsymbol{V}_{i j, k}}{\left(p_{i}+p_{j}\right)^{2}-m_{i j}^{2}} \frac{1}{\boldsymbol{T}_{i j}^{2}}\left|\mathcal{M}_{m}^{i j, k}\left(p_{1}, \ldots, \widetilde{p}_{i j}, \ldots, \widetilde{p}_{k}, \ldots, p_{m+1}\right)\right|^{2}\right]_{h}
$$

where $\mathcal{M}_{m}^{i j, k}$ is a colour-correlated $m$-parton amplitude (see Eq. (3.4)) depending only on $p_{1}, \ldots \widetilde{p}_{i j}, \widetilde{p}_{k}, \ldots, p_{m+1}$ while $\boldsymbol{V}_{i j, k} /\left[\left(p_{i}+p_{j}\right)^{2}-m_{i j}^{2}\right]$ depends only on $p_{i}, p_{j}, p_{k}$ or, equivalently, $p_{i}, \widetilde{p}_{i j}, \widetilde{p}_{k}$. The subscript $h$ on the square bracket in Eq. (6.11) means that $\boldsymbol{V}_{i j, k}$ and $\mathcal{M}_{m}^{i j, k}$ are still coupled in helicity space. According to the discussion in Sect. 5.1.3, this helicity-space coupling vanishes after integration over $\left[\mathrm{d} p_{i}\left(\widetilde{p}_{i j}, \widetilde{p}_{k}\right)\right]$, and the integral of the subtraction term remains the same if we exchange the splitting matrices for the azimuthally averaged splitting matrices. Then we can perform the counting of the symmetry factors in order to change $S_{\{m+1\}}$ to $S_{\{m\}}$, which follows exactly the procedure of the massless case[11] leading to Eq. (6.10) rewritten in a completely factorized form of an $m$-parton contribution multiplied by a singular integral:

$$
\begin{aligned}
\int_{m+1} \mathrm{~d} \sigma^{\mathrm{A}} & =-\mathcal{N}_{i n} \sum_{\{m\}} \int_{m} \mathrm{~d} \phi_{m}\left(p_{1}, \ldots, p_{m} ; Q\right) \frac{1}{S_{\{m\}}} F_{J}^{(m)}\left(p_{1}, \ldots, p_{m}\right) \\
& \times \sum_{j} \sum_{k \neq j} \frac{1}{\boldsymbol{T}_{j}^{2}}\left|\mathcal{M}_{m}^{j, k}\left(p_{1}, \ldots, p_{m}\right)\right|^{2}\left(\int\left[\mathrm{d} p_{i}\left(\widetilde{p}_{i j}, \widetilde{p}_{k}\right)\right] \frac{\left\langle\mathbf{V}_{i j, k}\right\rangle}{\left(p_{i}+p_{j}\right)^{2}-m_{i j}^{2}}\right)_{\substack{i j \rightarrow j \\
\tilde{k} \rightarrow k}} .
\end{aligned}
$$

The substitutions in the last factor are to be performed after the integral is calculated using the results in Sect. 5.1.3. In this context we observe that the colour-connected $m$ parton amplitude, $\mathcal{M}_{m}^{j, k}$ is symmetric in its upper indices, and we sum over all pairs of $j, k$ in Eq. 6.12). Consequently, the antisymmetric part of the eikonal integral, $I_{-}^{\text {eik }}$ does not contribute to the integral of $\mathrm{d} \sigma^{\mathrm{A}}$. 
After these manipulations, the final result for $\int_{m+1} \mathrm{~d} \sigma^{\mathrm{A}}$ in Eq. (6.12) can be written as follows,

$$
\int_{m+1} \mathrm{~d} \sigma^{\mathrm{A}}=\int_{m}\left[\mathrm{~d} \sigma^{\mathrm{B}} \otimes \boldsymbol{I}_{m}\left(\epsilon, \mu^{2} ;\left\{p_{i}, m_{i}\right\}\right)\right]
$$

where the notation $\left[\mathrm{d} \sigma^{\mathrm{B}} \otimes \boldsymbol{I}_{m}\right]$ on the right-hand side means that one has to write down the expression for $\mathrm{d} \sigma^{\mathrm{B}}$ and then replace the corresponding matrix element squared at the Born level

$$
\left|\mathcal{M}_{m}\right|^{2}={ }_{m}\langle 1, \ldots, m|| 1, \ldots, m\rangle_{m}
$$

by

$$
{ }_{m}\left\langle 1, \ldots, m\left|\boldsymbol{I}_{m}\left(\epsilon, \mu^{2} ;\left\{p_{i}, m_{i}\right\}\right)\right| 1, \ldots, m\right\rangle_{m}
$$

where the insertion operator $\boldsymbol{I}_{m}$ depends on the colour charges, momenta and masses of the $m$ final-state partons in $\mathrm{d} \sigma^{\mathrm{B}}$ :

$$
\begin{aligned}
\boldsymbol{I}_{m}\left(\epsilon, \mu^{2} ;\left\{p_{i}, m_{i}\right\}\right)=-\frac{\alpha_{\mathrm{s}}}{2 \pi} \frac{(4 \pi)^{\epsilon}}{\Gamma(1-\epsilon)} \sum_{j} \frac{1}{\boldsymbol{T}_{j}^{2}} \sum_{k \neq j} \boldsymbol{T}_{j} \cdot \boldsymbol{T}_{k} \\
\times\left[\boldsymbol{T}_{j}^{2}\left(\frac{\mu^{2}}{s_{j k}}\right)^{\epsilon}\left(\mathcal{V}_{j}\left(s_{j k}, m_{j}, m_{k},\left\{m_{F}\right\} ; \epsilon, \kappa\right)-\frac{\pi^{2}}{3}\right)+\Gamma_{j}\left(\mu, m_{j},\left\{m_{F}\right\} ; \epsilon\right)\right. \\
\left.\quad+\gamma_{j} \ln \frac{\mu^{2}}{s_{j k}}+\gamma_{j}+K_{j}+\mathrm{O}(\epsilon)\right]
\end{aligned}
$$

where $s_{j k}=2 p_{j} p_{k}$ and $\left\{m_{F}\right\}$ denotes the set of masses of those massive quarks that may appear in the $g \rightarrow q \bar{q}$ splitting. The constants $\gamma_{a}$, were defined in Eq. (5.91), while $K_{a}$ denote the constants introduced in Ref. [47],

$$
K_{q}=\left(\frac{7}{2}-\frac{\pi^{2}}{6}\right) C_{\mathrm{F}}, \quad K_{g}=\left(\frac{67}{18}-\frac{\pi^{2}}{6}\right) C_{\mathrm{A}}-\frac{10}{9} T_{\mathrm{R}} N_{f}
$$

In Eq. (6.16) the $\mathcal{V}_{j}$ kernels depend on the flavour of parton $j$ and on the momenta and masses of both partons $j$ and $k$. Thus colour correlations are accompanied by complicated mass correlations. We decompose these functions into a sum of two contributions: the first is symmetric with respect to interchange of the indices $j$ and $k$ and singular in four dimensions or for vanishing masses and the second is neither symmetric nor singular,

$$
\mathcal{V}_{j}\left(s_{j k}, m_{j}, m_{k},\left\{m_{F}\right\} ; \epsilon, \kappa\right)=\mathcal{V}^{(\mathrm{S})}\left(s_{j k}, m_{j}, m_{k} ; \epsilon\right)+\mathcal{V}_{j}^{(\mathrm{NS})}\left(s_{j k}, m_{j}, m_{k},\left\{m_{F}\right\} ; \kappa\right) .
$$

The singular terms have the following form:

$$
\mathcal{V}^{(\mathrm{S})}\left(s_{j k}, m_{j}, m_{k} ; \epsilon\right)=\frac{1}{v_{j k}}\left(\frac{Q_{j k}^{2}}{s_{j k}}\right)^{\epsilon}\left[\frac{1}{\epsilon^{2}}\left(1-\frac{1}{2} \rho_{j}^{-2 \epsilon}-\frac{1}{2} \rho_{k}^{-2 \epsilon}\right)-\frac{\pi^{2}}{12}\left(\Theta\left(m_{j}\right)+\Theta\left(m_{k}\right)\right)\right]
$$

where we introduced the abbreviation $Q_{j k}^{2} \equiv s_{j k}+m_{j}^{2}+m_{k}^{2}$ and $\Theta(x)$ is the step function with $\Theta(0)=0$. Here and in the following the quantities $\rho, \rho_{j}$ and $\rho_{k}$ are obtained from Eq. (5.30) with the substitutions $\mu_{n}^{2} \rightarrow m_{n}^{2} / Q_{j k}^{2}(n=j, k)$ and $\tilde{v}_{i j, k} \rightarrow v_{j k}$. In practice the 
expanded forms of $\mathcal{V}^{(\mathrm{S})}$ are needed,

$$
\begin{aligned}
\mathcal{V}^{(\mathrm{S})}\left(s_{j k}, m_{j}>0, m_{k}>0 ; \epsilon\right)= & \frac{1}{v_{j k}}\left[\frac{1}{\epsilon} \ln \rho-\frac{1}{4} \ln ^{2} \rho_{j}^{2}-\frac{1}{4} \ln ^{2} \rho_{k}^{2}-\frac{\pi^{2}}{6}\right] \\
& +\frac{1}{v_{j k}} \ln \rho \ln \left(\frac{Q_{j k}^{2}}{s_{j k}}\right), \\
\mathcal{V}^{(\mathrm{S})}\left(s_{j k}, m_{j}>0,0 ; \epsilon\right)= & \frac{1}{2 \epsilon^{2}}+\frac{1}{2 \epsilon} \ln \frac{m_{j}^{2}}{s_{j k}}-\frac{1}{4} \ln ^{2} \frac{m_{j}^{2}}{s_{j k}}-\frac{\pi^{2}}{12} \\
& -\frac{1}{2} \ln \frac{m_{j}^{2}}{s_{j k}} \ln \frac{s_{j k}}{Q_{j k}^{2}}-\frac{1}{2} \ln \frac{m_{j}^{2}}{Q_{j k}^{2}} \ln \frac{s_{j k}}{Q_{j k}^{2}} \\
\mathcal{V}^{(\mathrm{S})}\left(s_{j k}, 0,0 ; \epsilon\right)= & \frac{1}{\epsilon^{2}} .
\end{aligned}
$$

Of course, the decomposition defined in Eq. (6.18) is not unique. One can still shift finite terms between the non-singular and singular terms. In particular, in Ref. [31] we have not included those terms in the singular parts that vanish for vanishing masses (terms in the second lines of the equations in $(\underline{6.20}))$.

The non-singular terms depend on the flavours and masses of partons $j$ and $k$. If $j$ is a quark, then $\mathcal{V}_{q}^{(\mathrm{NS})}$ does not depend on $\left\{m_{F}\right\}$ and $\kappa$, so we suppress these arguments. If both $j$ and $k$ are massive quarks, then

$$
\begin{aligned}
\mathcal{V}_{q}^{(\mathrm{NS})} & \left(s_{j k}, m_{j}, m_{k}\right)=\frac{\gamma_{q}}{\boldsymbol{T}_{q}^{2}} \ln \frac{s_{j k}}{Q_{j k}^{2}} \\
& +\frac{1}{v_{j k}}\left[\ln \rho^{2} \ln \left(1+\rho^{2}\right)+2 \operatorname{Li}_{2}\left(\rho^{2}\right)-\mathrm{Li}_{2}\left(1-\rho_{j}^{2}\right)-\operatorname{Li}_{2}\left(1-\rho_{k}^{2}\right)-\frac{\pi^{2}}{6}\right] \\
& +\ln \frac{Q_{j k}-m_{k}}{Q_{j k}}-2 \ln \frac{\left(Q_{j k}-m_{k}\right)^{2}-m_{j}^{2}}{Q_{j k}^{2}}-\frac{2 m_{j}^{2}}{s_{j k}} \ln \frac{m_{j}}{Q_{j k}-m_{k}} \\
& -\frac{m_{k}}{Q_{j k}-m_{k}}+\frac{2 m_{k}\left(2 m_{k}-Q_{j k}\right)}{s_{j k}}+\frac{\pi^{2}}{2}
\end{aligned}
$$

where we used the abbreviation $Q_{j k}=\sqrt{Q_{j k}^{2}}$. If $j$ is a massive quark, but $k$ is a massless parton, this reduces to

$$
\mathcal{V}_{q}^{(\mathrm{NS})}\left(s_{j k}, m_{j}, 0\right)=\frac{\gamma_{q}}{\boldsymbol{T}_{q}^{2}} \ln \frac{s_{j k}}{Q_{j k}^{2}}+\frac{\pi^{2}}{6}-\mathrm{Li}_{2}\left(\frac{s_{j k}}{Q_{j k}^{2}}\right)-2 \ln \frac{s_{j k}}{Q_{j k}^{2}}-\frac{m_{j}^{2}}{s_{j k}} \ln \frac{m_{j}^{2}}{Q_{j k}^{2}} .
$$

If $j$ is a massless quark and $k$ is a massive parton, then

$$
\mathcal{V}_{q}^{(\mathrm{NS})}\left(s_{j k}, 0, m_{k}\right)=\frac{\gamma_{q}}{\boldsymbol{T}_{q}^{2}}\left[\ln \frac{s_{j k}}{Q_{j k}^{2}}-2 \ln \frac{Q_{j k}-m_{k}}{Q_{j k}}-\frac{2 m_{k}}{Q_{j k}+m_{k}}\right]+\frac{\pi^{2}}{6}-\operatorname{Li}_{2}\left(\frac{s_{j k}}{Q_{j k}^{2}}\right)
$$

If $j$ is a gluon, then

$$
\begin{aligned}
& \mathcal{V}_{j}^{(\mathrm{NS})}\left(s_{j k}, 0, m_{k},\left\{m_{F}\right\} ; \kappa\right)= \\
& \quad \frac{\gamma_{g}}{\boldsymbol{T}_{g}^{2}}\left[\ln \frac{s_{j k}}{Q_{j k}^{2}}-2 \ln \frac{Q_{j k}-m_{k}}{Q_{j k}}-\frac{2 m_{k}}{Q_{j k}+m_{k}}\right]+\frac{\pi^{2}}{6}-\operatorname{Li}_{2}\left(\frac{s_{j k}}{Q_{j k}^{2}}\right)
\end{aligned}
$$




$$
\begin{array}{r}
+\frac{4}{3} \frac{T_{\mathrm{R}}}{C_{\mathrm{A}}} \sum_{F=1}^{N_{F}^{j k}}\left[\ln \frac{Q_{j k}-m_{k}}{Q_{j k}}+\frac{m_{k} \rho_{1}^{3}}{Q_{j k}+m_{k}}+\ln \frac{1+\rho_{1}}{2}-\frac{\rho_{1}}{3}\left(3+\rho_{1}^{2}\right)-\frac{1}{2} \ln \frac{m_{F}^{2}}{Q_{j k}^{2}}\right] \\
+\frac{2}{3} \frac{T_{\mathrm{R}}}{C_{\mathrm{A}}} \sum_{F=1}^{N_{F}} \ln \frac{m_{F}^{2}}{Q_{\mathrm{aux}}^{2}}+\left(\kappa-\frac{2}{3}\right) \frac{m_{k}^{2}}{s_{j k}}\left[\left(2 \frac{T_{\mathrm{R}}}{C_{\mathrm{A}}} N_{f}-1\right) \ln \frac{2 m_{k}}{Q_{j k}+m_{k}}\right. \\
\left.+2 \frac{T_{\mathrm{R}}}{C_{\mathrm{A}}} \sum_{F=1}^{N_{F}^{j k}}\left(\rho_{2}^{3} \ln \left(\frac{\rho_{2}-\rho_{1}}{\rho_{2}+\rho_{1}}\right)-\ln \left(\frac{1-\rho_{1}}{1+\rho_{1}}\right)-\frac{8 \rho_{1} m_{F}^{2}}{s_{j k}}\right)\right],
\end{array}
$$

where $N_{F}$ is the number of heavy flavours and $N_{F}^{j k}$ is the number of those heavy flavours for which $s_{j k}>4 m_{F}\left(m_{F}+m_{k}\right)$. Note that for $m_{F} \rightarrow 0$, we can set $N_{F}^{j k}=N_{F}$ because the region of vanishing $s_{j k}$ gives a vanishing contribution to infrared-safe observables. Thus $\mathcal{V}_{j}^{(\mathrm{NS})}\left(s_{j k}, 0, m_{k},\left\{m_{F}\right\} ; \kappa\right)$ is finite in the massless limit. The auxiliary mass scale $Q_{\text {aux }}$ has been introduced only to ensure this finiteness property. It can be chosen arbitrarily will cancel against a similar contribution in $\Gamma_{g}\left(\left\{m_{F}\right\} ; \epsilon\right)$ (cf. Eq. (6.27)). In Eq. 66.24) $\rho_{1}$ and $\rho_{2}$ as defined in Eq. (5.38) can be rewritten as

$$
\rho_{1}=\sqrt{1-\frac{4 m_{F}^{2}}{\left(Q_{j k}-m_{k}\right)^{2}}}, \quad \rho_{2}=\sqrt{1-\frac{4 m_{F}^{2}}{Q_{j k}^{2}-m_{k}^{2}}} .
$$

We see that choosing $\kappa=2 / 3$ simplifies Eq. (6.24) considerably.

Finally, if both $j$ and $k$ are massless partons, then $\mathcal{V}_{q}^{(\mathrm{NS})}\left(s_{j k}, 0,0\right)=0$ and Eq. (6.24) reduces to

$$
\begin{aligned}
& \mathcal{V}_{g}^{(\mathrm{NS})}\left(s_{j k}, 0,0,\left\{m_{F}\right\}\right)= \\
& \quad \frac{4}{3} \frac{T_{\mathrm{R}}}{C_{\mathrm{A}}} \sum_{F=1}^{N_{F}^{j k}}\left[\ln \frac{1+\rho_{1}}{2}-\frac{\rho_{1}}{3}\left(3+\rho_{1}^{2}\right)-\frac{1}{2} \ln \frac{m_{F}^{2}}{s_{j k}}\right]+\frac{2}{3} \frac{T_{\mathrm{R}}}{C_{\mathrm{A}}} \sum_{F=1}^{N_{F}} \ln \frac{m_{F}^{2}}{Q_{\mathrm{aux}}^{2}},
\end{aligned}
$$

where we dropped $\kappa$ from the arguments because the dependence on $\kappa$ in Eq. (6.24) is proportional to the mass of parton $k$. If the heavy partons are completely absent, then $\mathcal{V}_{g}^{(\mathrm{NS})}\left(s_{j k}, 0,0,\{\}\right)=0$, with \{\} meaning the empty set.

The functions $\Gamma_{j}$ depend on the flavour of the parton $j$ and on the parton masses. In the case of gluons and massless quarks (antiquarks) we have 45] (with dummy arguments suppressed)

$$
\begin{aligned}
\Gamma_{g}\left(\left\{m_{F}\right\} ; \epsilon\right) & =\frac{1}{\epsilon} \gamma_{g}-\frac{2}{3} T_{\mathrm{R}} \sum_{F=1}^{N_{F}} \ln \frac{m_{F}^{2}}{Q_{\mathrm{aux}}^{2}}, \\
\Gamma_{q}(\epsilon) & =\frac{1}{\epsilon} \gamma_{q},
\end{aligned}
$$

while for massive quarks (antiquarks) we find

$$
\Gamma_{q}\left(\mu, m_{q} ; \epsilon\right)=\boldsymbol{T}_{q}^{2}\left(\frac{1}{\epsilon}-\ln \frac{m_{q}^{2}}{\mu^{2}}-2\right)+\gamma_{q} \ln \frac{m_{q}^{2}}{\mu^{2}}=C_{\mathrm{F}}\left[\frac{1}{\epsilon}+\frac{1}{2} \ln \frac{m_{q}^{2}}{\mu^{2}}-2\right] .
$$

\footnotetext{
"In Ref. [31] $Q_{\text {aux }}$ was chosen to be the renormalization scale.
} 
In Eq. (6.27) the summation runs over the heavy flavours.

Using formulae (6.16)-(6.29), it is easy to verify that the $\boldsymbol{I}_{m}\left(\epsilon, \mu^{2} ;\left\{p_{i}, m_{i}\right\}\right)$ operator tends to the corresponding operator of the massless theory smoothly,

$$
\lim _{\left\{m_{i}\right\} \rightarrow 0} \boldsymbol{I}_{m}\left(\epsilon, \mu^{2} ;\left\{p_{i}, m_{i}\right\}\right)=\boldsymbol{I}(\epsilon)+\mathrm{O}(\epsilon),
$$

where $\boldsymbol{I}(\epsilon)$ is defined in Eq. (7.26) of Ref. [11].

The NLO QCD cross section in Eq. (2.3) is finite for infrared-safe jet observables. We constructed the subtraction terms such that the first term is not only finite independently of the parton masses, but it also tends to the corresponding massless expressions smoothly if $F_{J}$ is quasi-collinear safe (see Eq. (2.6)). Therefore, the second term, spelled out in Eq. (6.8), also has to be finite and, using Eq. (6.30), tends smoothly to the corresponding massless expressions in the zero-mass limit. Thus all the $\epsilon$ poles and $\ln \left(m_{i}\right)$ terms that are singular in the zero-mass limit in $\mathrm{d} \sigma^{\mathrm{V}}$ must be cancelled by corresponding terms in $\mathrm{d} \sigma^{\mathrm{B}} \otimes \boldsymbol{I}_{m}(\epsilon)$, which enables us to write down the singular terms of the one-loop amplitudes, as spelled out explicitly in Ref. [31.

\subsection{Jet cross sections with one initial-state hadron}

As mentioned before, in this paper we consider only massless partons in the initial state, therefore, the implementation of the subtraction procedure for processes with hadrons in the initial state is the same as described in Ref. [1] (see also Sect. (6.1)). The presence of the parton masses induces non-trivial changes in the evaluation of the sum of the integral of the full subtraction term and the collinear counterterm,

$$
\int_{m+1} \mathrm{~d} \sigma_{a}^{\mathrm{A}}\left(p_{a}\right)+\int_{m} \mathrm{~d} \sigma_{a}^{\mathrm{C}}\left(p_{a}\right)
$$

where the index $a$ refers to the flavour of the incoming parton and $p_{a}$ to its momentum. The full subtraction term $\mathrm{d} \sigma_{a}^{\mathrm{A}}\left(p_{a}\right)$ is a sum of three terms, corresponding to the first three terms in Eq. (5.1),

$$
\mathrm{d} \sigma_{a}^{\mathrm{A}}\left(p_{a}\right)=\mathrm{d} \sigma_{a}^{\mathrm{A}^{\prime}}\left(p_{a}\right)+\mathrm{d} \sigma_{a}^{\mathrm{A}^{\prime \prime}}\left(p_{a}\right)+\mathrm{d} \sigma_{a}^{\mathrm{A}^{\prime \prime \prime}}\left(p_{a}\right) .
$$

The first term on the right-hand side is the subtraction term for final-state singularities with final-state spectators and has the same form as Eq. (6.10):

$$
\begin{array}{rl}
\int_{m+1} \mathrm{~d} \sigma_{a}^{\mathrm{A}^{\prime}}=\frac{\mathcal{N}_{i n}}{n_{s}(a) \mathcal{F}\left(p_{a}\right)} \sum_{\{m+1\}} \sum_{\substack{\text { pairs } \\
i, j}} \sum_{k \neq i, j} \int_{m} & \mathrm{~d} \phi_{m}\left(p_{1}, \ldots, \widetilde{p}_{i j}, \ldots, \widetilde{p}_{k}, \ldots, p_{m+1} ; p_{a}+Q\right) \\
& \times \frac{1}{S_{\{m+1\}}} F_{J}^{(m)}\left(p_{1}, \ldots, \widetilde{p}_{i j}, \ldots, \widetilde{p}_{k}, \ldots, p_{m+1} ; p_{a}\right) \\
& \times \int\left[\mathrm{d} p_{i}\left(\widetilde{p}_{i j}, \widetilde{p}_{k}\right)\right] \mathcal{D}_{i j, k}\left(p_{1}, \ldots, p_{m+1} ; p_{a}\right),
\end{array}
$$

where the flux factor fulfils the following scaling property:

$$
\mathcal{F}\left(\eta p_{a}\right)=\eta \mathcal{F}\left(p_{a}\right) .
$$


The integration of this term follows the steps of the previous section and leads to the analogous result, which, for later purposes, we write in a different form:

$$
\int_{m+1} \mathrm{~d} \sigma_{a}^{\mathrm{A}^{\prime}}=\sum_{a^{\prime}} \int_{0}^{1} \mathrm{~d} x \int_{m}\left[\mathrm{~d} \sigma_{a^{\prime}}^{\mathrm{B}}\left(x p_{a}\right) \otimes\left(\delta^{a a^{\prime}} \delta(1-x) \boldsymbol{I}_{m}(\epsilon)\right)\right]
$$

with $\boldsymbol{I}_{m}(\epsilon)$ given in (6.16).

The second term on the right-hand side of Eq. (6.32) is the subtraction term for finalstate singularities with initial-state spectators. We have seen in Eq. (5.46) that the phasespace factorization leads to a convolution over the boost parameter $x$, thus

$$
\begin{array}{rl}
\int_{m+1} \mathrm{~d} \sigma_{a}^{\mathrm{A}^{\prime \prime}}=\frac{\mathcal{N}_{i n}}{n_{s}(a) \mathcal{F}\left(p_{a}\right)} \sum_{\{m+1\}} \sum_{\substack{\text { pairs } \\
i, j}} \int_{0}^{1} & \mathrm{~d} x \int \mathrm{d} \phi_{m}\left(p_{1}, \ldots, \widetilde{p}_{i j}, \ldots, p_{m+1} ; x p_{a}+Q\right) \\
& \times \frac{1}{S_{\{m+1\}}} F_{J}^{(m)}\left(p_{1}, \ldots, \widetilde{p}_{i j}, \ldots, p_{m+1} ; \tilde{p}_{a}\right) \\
& \times \int\left[\mathrm{d} p_{i}\left(\widetilde{p}_{i j} ; p_{a}, x\right)\right] \mathcal{D}_{i j}^{a}\left(p_{1}, \ldots, p_{m+1} ; p_{a}\right) .
\end{array}
$$

Using the definition of the dipole function in Eq. (5.40), following the same manipulations as for the integral of the $\mathrm{d} \sigma^{\mathrm{A}^{\prime}}$ term and finally making use of the scaling property in Eq. (6.34), we find that Eq. (6.36) can be recast in the following form:

$$
\int_{m+1} \mathrm{~d} \sigma_{a}^{\mathrm{A}^{\prime \prime}}=\sum_{a^{\prime}} \int_{0}^{1} \mathrm{~d} x \int_{m}\left[\mathrm{~d} \sigma_{a^{\prime}}^{\mathrm{B}}\left(x p_{a}\right) \otimes\left(\delta^{a a^{\prime}} \boldsymbol{I}_{m, a^{\prime}}(x ; \epsilon)\right)\right] .
$$

The new insertion operator is

$$
\boldsymbol{I}_{m, a^{\prime}}\left(x ; \epsilon, \mu^{2} ;\left\{p_{i}, m_{i}\right\}, p_{a}\right)=-\frac{\alpha_{\mathrm{s}}}{2 \pi} \frac{(4 \pi)^{\epsilon}}{\Gamma(1-\epsilon)} \sum_{j} \boldsymbol{T}_{j} \cdot \boldsymbol{T}_{a^{\prime}}\left(\frac{\mu^{2}}{s_{j a}}\right)^{\epsilon} \frac{1}{\boldsymbol{T}_{j}^{2}} \mathcal{V}_{j}\left(x ; s_{j a}, m_{j},\left\{m_{F}\right\} ; \epsilon\right)
$$

where $s_{j a}=2 p_{j} p_{a}$. We emphasize that $p_{a}$ is the original initial-state momentum of the incoming parton, while the final-state momentum $p_{j}$ belongs to the boosted phase space with incoming momentum $x p_{a}$. The flavour kernel for a massive quark (or antiquark) $j$ is

$$
\begin{aligned}
& \mathcal{V}_{q}\left(x ; s_{j a}, m_{q},\{\} ; \epsilon\right)=C_{\mathrm{F}}\left[J_{g Q}^{a}\left(x, m_{q} / \sqrt{s_{j a}}\right)\right]_{+}+\delta(1-x)\left[\Gamma_{q}\left(\sqrt{s_{j a}}, m_{q} ; \epsilon\right)+K_{q}\right] \\
& \quad+\delta(1-x) C_{\mathrm{F}}\left[\frac{1}{\epsilon} \ln \frac{m_{q}^{2}}{s_{j a}+m_{q}^{2}}-\frac{1}{2} \ln ^{2} \frac{m_{q}^{2}}{s_{j a}}-\frac{\pi^{2}}{2}+J_{g Q}^{a ; \mathrm{NS}}\left(\frac{m_{q}}{\sqrt{s_{j a}}}\right)\right] .
\end{aligned}
$$

The function $\Gamma_{q}\left(\sqrt{s_{j a}}, m_{q} ; \epsilon\right)$ is that defined in Eq. (6.29) with the $\mu \rightarrow \sqrt{s_{j a}}$ substitution. Here and in the following, \{\} denotes the empty set. For a massless quark $j=q$ the flavour kernel is given by

$$
\mathcal{V}_{q}\left(x ; s_{j a}, 0,\{\} ; \epsilon\right)=C_{\mathrm{F}}\left[J_{g Q}^{a}(x, 0)\right]_{+}+\delta(1-x)\left[C_{\mathrm{F}}\left(\frac{1}{\epsilon^{2}}-\frac{\pi^{2}}{3}\right)+\Gamma_{q}(\epsilon)+K_{q}\right] .
$$

If $j$ is a gluon, then

$$
\mathcal{V}_{g}\left(x ; s_{j a}, 0,\left\{m_{F}\right\} ; \epsilon\right)=C_{\mathrm{A}}\left[J_{g g}^{a}(x)\right]_{+}+T_{\mathrm{R}} N_{f}\left[J_{Q \bar{Q}}^{a}(x, 0)\right]_{+}
$$




$$
\begin{aligned}
& +\delta(1-x)\left[C_{\mathrm{A}}\left(\frac{1}{\epsilon^{2}}-\frac{\pi^{2}}{3}\right)+\Gamma_{g}\left(\left\{m_{F}\right\} ; \epsilon\right)+K_{g}\right] \\
& +T_{\mathrm{R}} \sum_{F=1}^{N_{F}^{j a}}\left\{\left[J_{Q \bar{Q}}^{a}\left(x, \frac{m_{F}}{\sqrt{s_{j a}}}\right)\right]_{x_{+}}+\delta\left(x_{+}-x\right)\left[J_{Q \bar{Q}}^{a ; \mathrm{NS}}\left(\frac{m_{F}}{\sqrt{s_{j a}}}\right)-\frac{10}{9}\right]\right\} \\
& +\frac{2}{3} T_{\mathrm{R}}\left[\delta(1-x) \sum_{F=1}^{N_{F}} \ln \frac{m_{F}^{2}}{Q_{\mathrm{aux}}^{2}}-\delta\left(x_{+}-x\right) \sum_{F=1}^{N_{F}^{j a}} \ln \frac{m_{F}^{2}}{s_{j a}}\right]
\end{aligned}
$$

where $x_{+}=1-4 m_{F}^{2} / s_{j a}$. Note that the last line, containing the difference of two $\delta$ functions, gives a contribution to Eq. (6.37) that can easily be calculated and has a smooth massless limit, since $N_{F}^{j a}$ can be replaced by $N_{F}$ as $m_{F} \rightarrow 0$.

The third term on the right-hand side of Eq. (6.32) is the subtraction term for initialstate singularities with final-state spectators. The relevant phase-space factorization, given in Eq. (5.46), is again a convolution,

$$
\begin{array}{rl}
\int_{m+1} \mathrm{~d} \sigma_{a}^{\mathrm{A}^{\prime \prime \prime}}=\frac{\mathcal{N}_{i n}}{n_{s}(a) \mathcal{F}\left(p_{a}\right)} \sum_{\{m+1\}} \sum_{i} \sum_{j \neq i} \int_{0}^{1} & \mathrm{~d} x \int \mathrm{d} \phi_{m}\left(p_{1}, \ldots, \tilde{p}_{j}, \ldots, p_{m+1} ; x p_{a}+Q\right) \\
& \times \frac{1}{S_{\{m+1\}}} F_{J}^{(m)}\left(p_{1}, \ldots, \tilde{p}_{j}, \ldots, p_{m+1} ; \tilde{p}_{a i}\right) \\
& \times \int\left[\mathrm{d} p_{i}\left(\tilde{p}_{j} ; p_{a}, x\right)\right] \mathcal{D}_{j}^{a i}\left(p_{1}, \ldots, p_{m+1} ; p_{a}\right) .
\end{array}
$$

The evaluation of the integral over $\left[\mathrm{d} p_{i}\left(\tilde{p}_{j} ; p_{a}, x\right)\right]$ follows that in the massless case, using the explicit results in Sect. 5.3.3. Then the counting of the symmetry factors is also straightforward and using the scaling property in Eq. (6.34), we find that Eq. (6.42) can be recast in the following form:

$$
\int_{m+1} \mathrm{~d} \sigma_{a}^{\mathrm{A}^{\prime \prime \prime}}=\sum_{a^{\prime}} \int_{0}^{1} \mathrm{~d} x \int_{m}\left[\mathrm{~d} \sigma_{a^{\prime}}^{\mathrm{B}}\left(x p_{a}\right) \otimes \boldsymbol{I}_{m, a a^{\prime}}(x ; \epsilon)\right]
$$

where the insertion operator is

$$
\boldsymbol{I}_{m, a a^{\prime}}\left(x ; \epsilon, \mu^{2} ;\left\{p_{i}, m_{i}\right\}, p_{a}\right)=-\frac{\alpha_{\mathrm{s}}}{2 \pi} \frac{(4 \pi)^{\epsilon}}{\Gamma(1-\epsilon)} \sum_{j} \boldsymbol{T}_{j} \cdot \boldsymbol{T}_{a^{\prime}}\left(\frac{\mu^{2}}{s_{j a}}\right)^{\epsilon} \frac{1}{\boldsymbol{T}_{a^{\prime}}^{2}} \mathcal{V}^{a, a^{\prime}}\left(x ; s_{j a}, m_{j} ; \epsilon\right)
$$

The functions $\mathcal{V}^{a, a^{\prime}}\left(x ; s_{j a}, m_{j} ; \epsilon\right)$ are related to the $I_{j}^{a b}\left(x ; \mu_{j} ; \epsilon\right)$ functions given in Eq. (5.88),

$$
\mathcal{V}^{a, a^{\prime}}\left(x ; s_{j a}, m_{j} ; \epsilon\right)=I_{j}^{a a^{\prime}}\left(x ; \frac{m_{j}}{\sqrt{s_{j a}}} ; \epsilon\right) .
$$

Note that $\mathrm{d} \sigma_{a}^{\mathrm{A}^{\prime \prime \prime}}$ is the only contribution to $\mathrm{d} \sigma_{a}^{\mathrm{A}}$ in $(6.32)$ where the flavours of the partons $a$ and $a^{\prime}$ can be different. However, both $a$ and $a^{\prime}$ are massless, since we do not allow for massive partons in the initial state. The fact that $\mathcal{V}^{g, a^{\prime}}$ does not receive contributions from the splitting $g \rightarrow Q \bar{Q}$ into massive quarks $Q$ is not a restriction of the presented formalism, it simply means that the logarithmic corrections $\alpha_{\mathrm{s}} \ln m_{Q}$ of this origin are not extracted from the real correction $\mathrm{d} \sigma^{\mathrm{R}}$ in (2.2). These terms could be extracted by a further subtraction into suitably-defined parton distributions, as discussed at the end of Sect. 2.2. 
Next we sum Eqs. (6.35), 6.37), 6.43) and the well-known expression for the collinearsubtraction counterterm,

$$
d \sigma_{a}^{C}\left(p_{a} ; \mu_{F}^{2}\right)=-\frac{\alpha_{\mathrm{s}}}{2 \pi} \frac{(4 \pi)^{\epsilon}}{\Gamma(1-\epsilon)} \sum_{a^{\prime}} \int_{0}^{1} \mathrm{~d} x\left[-\frac{1}{\epsilon}\left(\frac{\mu^{2}}{\mu_{F}^{2}}\right)^{\epsilon} P^{a a^{\prime}}(x)+K_{\mathrm{F} . \mathrm{S} .}^{a a^{\prime}}(x)\right] \mathrm{d} \sigma_{a^{\prime}}^{\mathrm{B}}\left(x p_{a}\right),
$$

to obtain the sum of the full subtraction term and the collinear counterterm in Eq. (6.31). In Eq. (6.46) $\mu_{F}$ is the factorization scale and the functions $K_{\mathrm{FS} .}^{a a^{\prime}}(x)$ define the factorization scheme. The $\overline{\mathrm{MS}}$ scheme is defined by $K_{\mathrm{FS} .}^{a a^{\prime}}(x)=0$. More on Eq. (6.46) can be found e.g. in Sect. 6 of Ref. [11. We see that $\int_{m+1} \mathrm{~d} \sigma_{a}^{\mathrm{A}}\left(p_{a}\right)+\int_{m} \mathrm{~d} \sigma_{a}^{\mathrm{C}}\left(p_{a}\right)$ is obtained from the leading-order expression $\int_{m} \mathrm{~d} \sigma_{a}^{\mathrm{B}}\left(x p_{a}\right)$ by replacing the leading-order matrix element squared

$$
\frac{1}{n_{s}(a)}{ }_{m, a}\left\langle\ldots ; p_{a} \| \ldots ; p_{a}\right\rangle_{m, a}
$$

by

$$
\sum_{a^{\prime}} \frac{1}{n_{s}\left(a^{\prime}\right)} m, a^{\prime}\left\langle\ldots ; x p_{a}\left|\boldsymbol{I}^{a, a^{\prime}}(x ; \epsilon)\right| \ldots ; x p_{a}\right\rangle_{m, a^{\prime}}
$$

and performing the $x$-integration. Here the insertion operator $\boldsymbol{I}^{a, a^{\prime}}(x ; \epsilon)$ also depends on the renormalization and factorization scales, colour charges, momenta, masses and flavours of the QCD partons,

$$
\begin{aligned}
& \boldsymbol{I}^{a, a^{\prime}}\left(x ; \epsilon, \mu^{2}, \mu_{F}^{2} ;\left\{p_{i}, m_{i}\right\}, p_{a}\right)=\delta^{a a^{\prime}} \delta(1-x) \boldsymbol{I}_{m}\left(\epsilon, \mu^{2} ;\left\{p_{i}, m_{i}\right\}\right) \\
& \quad+\delta^{a a^{\prime}} \boldsymbol{I}_{m, a^{\prime}}\left(x ; \epsilon, \mu^{2} ;\left\{p_{i}, m_{i}\right\}, p_{a}\right)+\boldsymbol{I}_{m, a a^{\prime}}\left(x ; \epsilon, \mu^{2} ;\left\{p_{i}, m_{i}\right\}, p_{a}\right) \\
& \quad-\frac{\alpha_{\mathrm{s}}}{2 \pi} \frac{(4 \pi)^{\epsilon}}{\Gamma(1-\epsilon)}\left[-\frac{1}{\epsilon}\left(\frac{\mu^{2}}{\mu_{F}^{2}}\right)^{\epsilon} P^{a a^{\prime}}(x)+K_{\mathrm{FS}}^{a a^{\prime}}(x)\right]+\mathrm{O}(\epsilon) .
\end{aligned}
$$

One can understand the structure of the $\boldsymbol{I}^{a, a^{\prime}}$ insertion operator, if we rewrite it as

$$
\begin{gathered}
\boldsymbol{I}^{a, a^{\prime}}\left(x ; \epsilon, \mu^{2}, \mu_{F}^{2} ;\left\{p_{i}, m_{i}\right\}, p_{a}\right)=\delta^{a a^{\prime}} \delta(1-x) \boldsymbol{I}_{m+a}\left(\epsilon, \mu^{2} ;\left\{p_{i}, m_{i}\right\}, p_{a}\right) \\
+\boldsymbol{P}_{m}^{a, a^{\prime}}\left(x ; \mu_{F}^{2} ;\left\{p_{i}\right\}, x p_{a}\right)+\boldsymbol{K}_{m}^{a, a^{\prime}}\left(x ;\left\{p_{i}, m_{i}\right\}, p_{a}\right)+\mathrm{O}(\epsilon),
\end{gathered}
$$

where the operator $\boldsymbol{I}_{m+a}\left(\epsilon, \mu^{2} ;\left\{p_{i}, m_{i}\right\}, p_{a}\right)$ is similar to the operator defined in Eq. (6.16),

$$
\boldsymbol{I}_{m+a}\left(\epsilon, \mu^{2} ;\left\{p_{i}, m_{i}\right\}, p_{a}\right)=\boldsymbol{I}_{m}\left(\epsilon, \mu^{2} ;\left\{p_{i}, m_{i}\right\}\right)+\boldsymbol{I}_{a}\left(\epsilon, \mu^{2} ;\left\{p_{i}, m_{i}\right\}, p_{a}\right),
$$

where

$$
\begin{aligned}
& \boldsymbol{I}_{a}\left(\epsilon, \mu^{2} ;\left\{p_{i}, m_{i}\right\}, p_{a}\right)=-\frac{\alpha_{\mathrm{s}}}{2 \pi} \frac{(4 \pi)^{\epsilon}}{\Gamma(1-\epsilon)} \sum_{j}\{ \\
& \frac{1}{\boldsymbol{T}_{j}^{2}} \boldsymbol{T}_{j} \cdot \boldsymbol{T}_{a}\left[\boldsymbol{T}_{j}^{2}\left(\frac{\mu^{2}}{s_{j a}}\right)^{\epsilon}\left(\mathcal{V}_{j}\left(s_{j a}, m_{j}, 0,\left\{m_{F}\right\} ; \epsilon, \kappa\right)-\frac{\pi^{2}}{3}\right)+\Gamma_{j}\left(\mu, m_{j},\left\{m_{F}\right\} ; \epsilon\right)\right. \\
& \left.+\gamma_{j} \ln \frac{\mu^{2}}{s_{j a}}+\gamma_{j}+K_{j}\right] \\
& +\frac{1}{\boldsymbol{T}_{a}^{2}} \boldsymbol{T}_{a} \cdot \boldsymbol{T}_{j}\left[\boldsymbol{T}_{a}^{2}\left(\frac{\mu^{2}}{s_{a j}}\right)^{\epsilon}\left(\mathcal{V}_{a}\left(s_{a j}, 0, m_{j},\{\} ; \epsilon, 2 / 3\right)-\frac{\pi^{2}}{3}\right)+\frac{\gamma_{a}}{\epsilon}\right. \\
& \left.\left.+\gamma_{a} \ln \frac{\mu^{2}}{s_{a j}}+\gamma_{a}+K_{a}\right]\right\} \text {. }
\end{aligned}
$$


The only difference between $\boldsymbol{I}_{m}$ and $\boldsymbol{I}_{m+a}$ is that the latter depends on the additional initial-state parton $a$ such that in the functions $\mathcal{V}_{a}$ and $\Gamma_{a}$ the set $\left\{m_{F}\right\}$ is empty and $\kappa=2 / 3$. The singular terms $\mathcal{V}^{(\mathrm{S})}$ do not depend on the value of $\left\{m_{F}\right\}$ and $\kappa$, and crossing the momentum of a parton from the final to the initial state does not change the singular terms in the one-loop QCD amplitudes, therefore, this insertion operator cancels all the singularities in the virtual contribution $\int_{m} \mathrm{~d} \sigma_{a}^{\mathrm{V}}\left(p_{a}\right)$. As a result, the operators $\boldsymbol{P}_{m}^{a, a^{\prime}}$ and $\boldsymbol{K}_{m}^{a, a^{\prime}}$ are finite in four dimensions, as can also be seen from their explicit forms below.

The $\boldsymbol{P}_{m}^{a, a^{\prime}}$ operator, which is defined by

$$
\boldsymbol{P}_{m}^{a, a^{\prime}}\left(x ; \mu_{F}^{2} ;\left\{p_{i}\right\}, x p_{a}\right)=\frac{\alpha_{\mathrm{s}}}{2 \pi} P^{a a^{\prime}}(x) \frac{1}{\boldsymbol{T}_{a^{\prime}}^{2}} \sum_{j} \boldsymbol{T}_{j} \cdot \boldsymbol{T}_{a^{\prime}} \ln \frac{\mu_{F}^{2}}{x s_{j a}},
$$

is independent of the presence of massive partons and contains the full dependence on the factorization scale $\mu_{F}$. In order to obtain this form of the $\mu_{F}$ dependence, we have used colour conservation $\left(\sum_{j} \boldsymbol{T}_{j}=-\boldsymbol{T}_{a^{\prime}}\right)$ and expanded in $\epsilon$,

$$
\left[\sum_{j} \boldsymbol{T}_{j} \cdot \boldsymbol{T}_{a^{\prime}}\left(\frac{\mu^{2}}{s_{j a}}\right)^{\epsilon} \frac{1}{\boldsymbol{T}_{a^{\prime}}^{2}}+\left(\frac{\mu^{2}}{\mu_{F}^{2}}\right)^{\epsilon}\right] \frac{1}{\epsilon} P^{a a^{\prime}}(x)=P^{a a^{\prime}}(x) \frac{1}{\boldsymbol{T}_{a^{\prime}}^{2}} \sum_{j} \boldsymbol{T}_{j} \cdot \boldsymbol{T}_{a^{\prime}} \ln \frac{\mu_{F}^{2}}{s_{j a}}+\mathrm{O}(\epsilon) .
$$

The $\boldsymbol{K}_{m}^{a, a^{\prime}}$ operator contains all remaining terms, including the factorization-scheme dependence and further finite-mass corrections:

$$
\begin{aligned}
\boldsymbol{K}_{m}^{a, a^{\prime}}\left(x ;\left\{p_{i}, m_{i}\right\}, p_{a}\right) & =\frac{\alpha_{\mathrm{s}}}{2 \pi}\left\{\bar{K}^{a a^{\prime}}(x)-K_{\mathrm{F.S}}^{a a^{\prime}}(x)-\sum_{j} \boldsymbol{T}_{j} \cdot \boldsymbol{T}_{a^{\prime}} \mathcal{K}_{j}^{a, a^{\prime}}\left(x ; s_{j a}, m_{j},\left\{m_{F}\right\}\right)\right. \\
-\frac{1}{\boldsymbol{T}_{a^{\prime}}^{2}} \sum_{j} \boldsymbol{T}_{j} \cdot \boldsymbol{T}_{a^{\prime}}\left[P_{\mathrm{reg}}^{a a^{\prime}}(x) \ln \frac{(1-x) s_{j a}}{(1-x) s_{j a}+m_{j}^{2}}+\gamma_{a} \delta^{a a^{\prime}} \delta(1-x)\right. & \\
& \left.\left.\times\left(\ln \frac{s_{j a}-2 m_{j} \sqrt{s_{j a}+m_{j}^{2}}+2 m_{j}^{2}}{s_{j a}}+\frac{2 m_{j}}{\sqrt{s_{j a}+m_{j}^{2}}+m_{j}}\right)\right]\right\}
\end{aligned}
$$

Comparing the expressions for the various insertion operators on the right-hand side of Eq.(6.49), we observe that the finiteness of $\boldsymbol{K}_{m}^{a, a^{\prime}}$ is highly non-trivial: terms from the integration of all possible dipole contributions have to be combined, together with the collinear subtraction, before taking the $\epsilon \rightarrow 0$ limit.

In order to simplify the final formulae, in Eq. (6.55) we used the functions $P_{\text {reg }}^{a a^{\prime}}(x)$ and $P^{a a^{\prime}}(x)$, defined in Eqs. (5.89) and (5.94), respectively, and also the $\bar{K}^{a a^{\prime}}(x)$ functions defined in Ref. [11],

$$
\begin{aligned}
& \bar{K}^{a a^{\prime}}(x)=P_{\text {reg }}^{a a^{\prime}}(x) \ln \frac{1-x}{x}+\hat{P}^{\prime a a^{\prime}}(x) \\
& \quad+\delta^{a a^{\prime}}\left[\boldsymbol{T}_{a}^{2}\left(\frac{2}{1-x} \ln \frac{1-x}{x}\right)_{+}-\delta(1-x)\left(\gamma_{a}+K_{a}-\frac{5}{6} \pi^{2} \boldsymbol{T}_{a}^{2}\right)\right] .
\end{aligned}
$$

Recall that the functions $\hat{P}^{\prime a b}(x)$ come from the $\epsilon$-dependence of the splitting functions and are given in Eq. (5.93). 
The functions $\mathcal{K}_{q}^{a, a^{\prime}}\left(x ; s_{j a}, m_{j},\left\{m_{F}\right\}\right)$ depend on the flavours of partons $a, a^{\prime}$ and $j$. For the sake of clarity, we consider the various cases separately. If $j$ is a quark, then $\mathcal{K}_{q}^{a, a^{\prime}}$ does not depend on $\left\{m_{F}\right\}$ and we have

$$
\begin{aligned}
\mathcal{K}_{q}^{g, q}\left(x ; s_{j a}, m_{j}\right) & =0 \\
\mathcal{K}_{q}^{q, q}\left(x ; s_{j a}, m_{j}\right) & =2\left[\left(\frac{\ln (1-x)}{1-x}\right)_{+}-\frac{\ln (2-x)}{1-x}\right]+\left[J_{g Q}^{a}\left(x, \frac{m_{j}}{\sqrt{s_{j a}}}\right)\right]_{+} \\
& +2\left(\frac{1}{1-x}\right)_{+} \ln \frac{(2-x) s_{j a}}{(2-x) s_{j a}+m_{j}^{2}}-\frac{\gamma_{q}}{C_{\mathrm{F}}} \delta(1-x) \\
& +\delta(1-x)\left(\frac{m_{j}^{2}}{s_{j a}} \ln \frac{m_{j}^{2}}{s_{j a}+m_{j}^{2}}+\frac{1}{2} \frac{m_{j}^{2}}{s_{j a}+m_{j}^{2}}\right), \\
\mathcal{K}_{q}^{q, g}\left(x ; s_{j a}, m_{j}\right) & =2 \frac{C_{\mathrm{F}}}{C_{\mathrm{A}}} \frac{m_{j}^{2}}{x s_{j a}} \ln \frac{m_{j}^{2}}{(1-x) s_{j a}+m_{j}^{2}}, \\
\mathcal{K}_{q}^{g, g}\left(x ; s_{j a}, m_{j}\right) & =\mathcal{K}_{q}^{q, q}\left(x ; s_{j a}, m_{j}\right)+\frac{C_{\mathrm{A}}}{C_{\mathrm{F}}} \mathcal{K}_{q}^{q, g}\left(x ; s_{j a}, m_{j}\right),
\end{aligned}
$$

If $j$ is a gluon, then

$$
\begin{aligned}
& \mathcal{K}_{g}^{a, a^{\prime}}\left(x ; s_{j a}, 0,\right.\left.\left\{m_{F}\right\}\right)=-\delta^{a a^{\prime}} \frac{\gamma_{g}}{C_{\mathrm{A}}}\left[\left(\frac{1}{1-x}\right)_{+}+\delta(1-x)\right] \\
&+\delta^{a a^{\prime}} \frac{T_{\mathrm{R}}}{C_{\mathrm{A}}} \sum_{F=1}^{N_{F}^{j a}}\left\{\left(\delta(1-x)-\delta\left(x_{+}-x\right)\right)\left[\frac{2}{3}\left(\ln \frac{m_{F}^{2}}{s_{j a}}+\frac{5}{3}\right)-J_{Q \bar{Q}}^{a ; \mathrm{NS}}\left(\frac{m_{F}}{\sqrt{s_{j a}}}\right)\right]\right. \\
&\left.+\left[J_{Q \bar{Q}}^{a}\left(x, \frac{m_{F}}{\sqrt{s_{j a}}}\right)\right]_{x_{+}}+\delta(1-x) \frac{2}{3}\left(1-\frac{4 m_{F}^{2}}{s_{j a}}\right)^{3 / 2}\right\}
\end{aligned}
$$

where $x_{+}=1-4 m_{F}^{2} / s_{j a}$ and $N_{F}^{j a}$ is the number of flavours with $s_{j a}>4 m_{F}^{2}$. Note that in this case $m_{j}=0$, so the terms proportional to $1 / \boldsymbol{T}_{a^{\prime}}^{2}$ in Eq. (6.55) vanish. In the zero-mass limit the functions $\mathcal{K}_{j}^{a, a^{\prime}}$ simplify to

$$
\mathcal{K}_{j}^{a, a^{\prime}}\left(x ; s_{j a}, 0,\{\}\right)=-\delta^{a a^{\prime}} \frac{\gamma_{j}}{\boldsymbol{T}_{j}^{2}}\left[\left(\frac{1}{1-x}\right)_{+}+\delta(1-x)\right] .
$$

Using Eq. (6.62), we see immediately that in the massless limit the operator $\boldsymbol{K}_{m}^{a, a^{\prime}}$ tends to the corresponding massless operator smoothly,

$$
\lim _{\left\{m_{i}\right\} \rightarrow 0} \boldsymbol{K}_{m}^{a, a^{\prime}}\left(x ;\left\{p_{i}, m_{i}\right\}, p_{a}\right)=\boldsymbol{K}_{m}^{a, a^{\prime}}(x),
$$

where $\boldsymbol{K}_{m}^{a, a^{\prime}}(x)$ is the same operator as defined in Eq. (8.38) of Ref. 11.

Finally, we explain the actual evaluation of the '+'-distribution that contains the kinematic variable $s_{j a}$ in more detail. As mentioned at the end of Sect. 5.2.3, $s_{j a}$ has to be kept fixed during the $x$ integration if $s_{j a}$ appears inside the ' + '-prescription, as is the case in $\mathcal{K}_{j}^{a, b}$ for $a=b$. Schematically we have to evaluate an integral of the form

$$
\int_{0}^{1} \mathrm{~d} x \int \mathrm{d} \Phi(x)\left[J\left(x, s_{j a}^{(x)}\right)\right]_{+}|\mathcal{M}(\Phi(x))|^{2}
$$


where the label $(x)$ of $s_{j a}^{(x)}$ indicates that in caclulating this variable the final-state momentum $p_{j}$ belongs to the phase space $\Phi(x)$ of the $x$-boosted frame, but $p_{a}$ is the original initial-state momentum of the incoming parton. At first sight this seems to be very inconvenient, but in practice the procedure can be simplified according to

$$
\begin{gathered}
\int_{0}^{1} \mathrm{~d} x \int \mathrm{d} \Phi(x) \int_{-\infty}^{+\infty} \mathrm{d} \bar{s}_{j a} \delta\left(\bar{s}_{j a}-s_{j a}^{(x)}\right)\left[J\left(x, \bar{s}_{j a}\right)\right]_{+}|\mathcal{M}(\Phi(x))|^{2} \\
=\int_{0}^{1} \mathrm{~d} x \int_{-\infty}^{+\infty} \mathrm{d} \bar{s}_{j a} J\left(x, \bar{s}_{j a}\right)\left\{\int \mathrm{d} \Phi(x)|\mathcal{M}(\Phi(x))|^{2} \delta\left(\bar{s}_{j a}-s_{j a}^{(x)}\right)\right. \\
\left.-\int \mathrm{d} \Phi(1)|\mathcal{M}(\Phi(1))|^{2} \delta\left(\bar{s}_{j a}-s_{j a}^{(1)}\right)\right\} \\
=\int_{0}^{1} \mathrm{~d} x\left\{\int \mathrm{d} \Phi(x) J\left(x, s_{j a}^{(x)}\right)|\mathcal{M}(\Phi(x))|^{2}-\int \mathrm{d} \Phi(1) J\left(x, s_{j a}^{(1)}\right)|\mathcal{M}(\Phi(1))|^{2}\right\} .
\end{gathered}
$$

Note that the variable $s_{j a}$ that appears in the function $J\left(x, s_{j a}\right)$ is the one in the respective phase-space integral. A non-trivial change in the endpoint contribution occurs if this variable $s_{j a}$ is eliminated in favour of an $x$-dependent variable $f(x)$, such as $f(x)=m_{j}^{2}-x s_{j a}$ as described in App. B. More precisely, if $s_{j a}^{(1)}$ in Eq. (6.65) is replaced by $f(1)$, the endpoint changes; if $s_{j a}^{(1)}$ is replaced by $f(x)$ the endpoint remains unchanged.

\subsection{Jet cross sections with two initial-state hadrons}

The derivation of the insertion operators $\boldsymbol{I}_{m+a+b}, \boldsymbol{P}_{m+b}$ and $\boldsymbol{K}_{m+b}$, relevant for calculating the NLO correction of Eqs. (6.8) and (6.9), does not contain any new features as compared to the combination of arguments given in Sect. 10 of Ref. [11] and in the previous subsection. Therefore, we simply list the corresponding results.

The $\boldsymbol{I}_{m+a+b}$ insertion operator is again very similar to $\boldsymbol{I}_{m}$ of Eq. (6.16). The only difference is that it depends on the additional initial-state partons $a$ and $b$ such that in the functions $\mathcal{V}_{a(b)}$ and $\Gamma_{a(b)}$ the set $\left\{m_{F}\right\}$ is empty and $\kappa=2 / 3$, which does not influence the singularity structure:

$$
\begin{aligned}
& \boldsymbol{I}_{m+a+b}\left(\epsilon, \mu^{2} ;\left\{p_{i}, m_{i}\right\}, p_{a}, p_{b}\right)= \\
& \quad \boldsymbol{I}_{m}\left(\epsilon, \mu^{2} ;\left\{p_{i}, m_{i}\right\}\right)+\boldsymbol{I}_{a}\left(\epsilon, \mu^{2} ;\left\{p_{i}, m_{i}\right\}, p_{a}\right)+\boldsymbol{I}_{b}\left(\epsilon, \mu^{2} ;\left\{p_{i}, m_{i}\right\}, p_{b}\right) \\
& \quad-\frac{\alpha_{\mathrm{s}}}{2 \pi} \frac{(4 \pi)^{\epsilon}}{\Gamma(1-\epsilon)}\left(\frac{1}{\boldsymbol{T}_{a}^{2}} \boldsymbol{T}_{a} \cdot \boldsymbol{T}_{b}\left[\left(\frac{\mu^{2}}{s_{a b}}\right)^{\epsilon}\left(\frac{\boldsymbol{T}_{a}^{2}}{\epsilon^{2}}+\frac{\gamma_{a}}{\epsilon}\right)-\boldsymbol{T}_{a}^{2} \frac{\pi^{2}}{3}+\gamma_{a}+K_{a}\right]+(a \leftrightarrow b)\right) .
\end{aligned}
$$

The operator $\boldsymbol{P}_{m+b}$ is completely analogous to $\boldsymbol{P}_{m}$ of Eq. (6.53) apart from the trivial dependence on the additional initial-state parton,

$$
\boldsymbol{P}_{m+b}^{a, a^{\prime}}\left(x ; \mu_{F}^{2} ;\left\{p_{i}\right\}, x p_{a}, p_{b}\right)=\boldsymbol{P}_{m}^{a, a^{\prime}}\left(x ; \mu_{F}^{2} ;\left\{p_{i}\right\}, x p_{a}\right)+\frac{\alpha_{\mathrm{s}}}{2 \pi} P^{a a^{\prime}}(x) \frac{1}{\boldsymbol{T}_{a^{\prime}}^{2}} \boldsymbol{T}_{b} \cdot \boldsymbol{T}_{a^{\prime}} \ln \frac{\mu_{F}^{2}}{x s_{a b}}
$$

Finally, the $\boldsymbol{K}_{m+b}$ operator is

$$
\begin{aligned}
& \boldsymbol{K}_{m+b}^{a, a^{\prime}}\left(x ;\left\{p_{i}, m_{i}\right\}, p_{a}, p_{b}\right)=\boldsymbol{K}_{m}^{a, a^{\prime}}\left(x ;\left\{p_{i}, m_{i}\right\}, p_{a}\right) \\
& \quad-\frac{\alpha_{\mathrm{s}}}{2 \pi} \boldsymbol{T}_{b} \cdot \boldsymbol{T}_{a^{\prime}}\left\{\frac{1}{\boldsymbol{T}_{a^{\prime}}^{2}} P_{\mathrm{reg}}^{a a^{\prime}}(x) \ln (1-x)+\delta^{a a^{\prime}}\left[2\left(\frac{\ln (1-x)}{1-x}\right)_{+}-\frac{\pi^{2}}{3} \delta(1-x)\right]\right\} .
\end{aligned}
$$


We see that $\boldsymbol{K}_{m+b}$ has the form of $\boldsymbol{K}_{m}$ of Eq. (6.55) with the additional term in the second line containing parton-parton correlations between the initial-state partons.

\section{Summary}

In this paper, we have presented an extension of the dipole subtraction method for calculating arbitrary (phenomenologically relevant) jet cross sections at NLO accuracy in arbitrary scattering processes involving heavy partons in the final state. In the case of lepton collisions, we have set up the formalism such that for those cross sections for which the mass of the heavy parton does not set the hard-scattering scale, i.e. the massless limit is IR safe, we can simply set the masses of the partons to zero to recover the massless limit of the computations, discussed in great detail in Ref. [11]. Thus the implementation of our formalism into a general purpose NLO partonic Monte Carlo program leads to a code that is smooth in the massless limit or, more importantly, numerically stable for any values of the hard-scattering scale. In hadron collisions, similar behaviour can be achieved by matching the partonic calculation with a suitable definition of the heavy-parton distributions in the massless limit. This feature will be particularly important at the future colliders (LHC, NLC), where the ratios of parton masses to other relevant kinematical invariants can run over a very wide range of values.

The factorization of the QCD matrix elements on soft poles in the presence of massive partons has been known and used for a long time. In those kinematical situations in which two partons become collinear and at least one of them is massive, the collinear divergences in the matrix elements are screened by the finite value of the parton mass and the regularization of the real corrections in these regions is not, strictly speaking, necessary. Nevertheless, the cross section receives a logarithmically enhanced contribution from these phase space regions when the parton masses become small compared to other relevant kinematical invariants. In order to assure the smooth massless behaviour of the cross section, we introduced the notion of quasi-collinear limit and presented the factorization of the QCD matrix elements on quasi-collinear poles (factors that become real poles in the massless limit). The smooth massless limit also serves as a powerful check of both the analytic calculations and any numerical implementation.

Our extension is formulated very closely along the lines of Ref. [11]. We changed the formalism to the least extent that was necessary to incorporate the mass corrections. Technically the generalization is cumbersome, but the complications only concern the derivation of the insertion operators (done in this paper) and the actual application is not much more involved than it is in the massless case. As a result, an existing general purpose NLO Monte Carlo program, such as NLOJET++ 17, 18, written for computations in the massless theory, can be changed straightforwardly to incorporate parton masses. In particular, the general form of the dipole subtraction terms remains unchanged and the dipole splitting functions receive trivial mass corrections. Only the definition of the emitter and spectator momenta, needed for writing the matrix elements and jet functions of the subtraction term change in a somewhat cumbersome, but straightforward, way.

The NLO contribution containing the virtual corrections and the insertion operators also 
has the same form as in the massless case. We have presented these operators explicitly, thus, for constructing a numerical program to calculate the NLO corrections to arbitrary jet quantities in a given process, the only additional ingredients that we need are simply related to the evaluation of the original matrix elements, as listed in Sect. 2.3.

Acknowledgements: This work was supported in part by the EU Fourth Framework Programme "Training and Mobility of Researchers", Network "QCD and particle structure", contract FMRX-CT98-0194 (DG 12 - MIHT) and by the Hungarian Scientific Research Fund grants OTKA T-025482 and T-038240. SD and ZT are grateful for the kind hospitality of the CERN Theory Division, where much of this work was carried out. We are also grateful to Arnd Brandenburg for supplying maple files of the results of Ref. [59], which we used to derive Eq. (D.40).

\section{Appendix}

\section{A The eikonal integral}

In Sect. 5.1.3 we have defined the eikonal integral $I^{\text {eik }}\left(\mu_{j}, \mu_{k}\right)$ in Eq. (5.26), but did not give the general expression, since only the part symmetric in $\mu_{j} \leftrightarrow \mu_{k}$ is needed in the final result. An intermediate result of the general integral, which is given in the following, is

$$
\begin{aligned}
I^{\mathrm{eik}}\left(\mu_{j}, \mu_{k}\right)=\frac{1}{\tilde{v}_{i j, k}} & {\left[\left(1-\left(\mu_{j}+\mu_{k}\right)^{2}\right)^{-2 \epsilon} \frac{1}{2 \epsilon^{2}}\left(1-\rho_{j}^{-2 \epsilon}\right)+\frac{\pi^{2}}{12}\left(1-\rho_{j}^{-2 \epsilon}\right)\right.} \\
+ & \left.\operatorname{Li}_{2}\left(1-\left(\mu_{j}+\mu_{k}\right)^{2}\right)-\operatorname{Li}_{2}\left(1-\rho_{j}^{2}\right)-2 f\left(\mu_{j}, \mu_{k}\right)\right] \\
+ & \mathrm{O}(\epsilon)
\end{aligned}
$$

with $\rho_{j}, \rho_{k}, \rho$ defined in Eq. (5.30) and $f\left(\mu_{j}, \mu_{k}\right)$ is an auxiliary integral,

$$
f\left(\mu_{j}, \mu_{k}\right)=\int_{t_{-}}^{1} \mathrm{~d} t(1-t)^{-1} \ln \frac{1+\mu_{j}^{2}-\mu_{k}^{2}+\sqrt{\lambda\left(1, \mu_{j}^{2}, \mu_{k}^{2}\right)}}{t+\mu_{j}^{2}-\mu_{k}^{2}+\sqrt{\lambda\left(t, \mu_{j}^{2}, \mu_{k}^{2}\right)}}, \quad t_{-}=\left(\mu_{j}+\mu_{k}\right)^{2} .
$$

The explicit result for $f\left(\mu_{j}, \mu_{k}\right)$ reads

$$
\begin{aligned}
f\left(\mu_{j}, \mu_{k}\right)= & \operatorname{Li}_{2}\left(\frac{1-\rho_{j}^{2}}{1+\rho}\right)+\operatorname{Li}_{2}\left(\frac{\rho\left(\rho_{j}^{2}-1\right)}{(1+\rho) \rho_{j}^{2}}\right)+\operatorname{Li}_{2}\left(1-\rho_{j}^{2}\right)-\operatorname{Li}_{2}\left(-\frac{\mu_{k}}{\mu_{j}}\right)+\operatorname{Li}_{2}\left(-\frac{\mu_{k}}{\mu_{j} \rho}\right) \\
& -2 \ln \rho_{j} \ln \left(\frac{\rho_{k}}{1+\rho}\right)-\ln \rho \ln \left(1+\frac{\mu_{k}}{\mu_{j} \rho}\right),
\end{aligned}
$$

where the massless limit $\mu_{j} \rightarrow 0$ yields $f\left(0, \mu_{k}\right)=\pi^{2} / 6$.

The symmetric part of the integral defined in Eq. (A.2) is much simpler than the full result and is given by

$$
\frac{1}{2}\left[f\left(\mu_{j}, \mu_{k}\right)+f\left(\mu_{k}, \mu_{j}\right)\right]=\frac{1}{2} \operatorname{Li}_{2}\left(1-\left(\mu_{j}+\mu_{k}\right)^{2}\right)-\operatorname{Li}_{2}(-\rho)+\operatorname{Li}_{2}(1-\rho)-\frac{\pi^{2}}{12},
$$


with $\rho$ as defined in Eq. (5.30). We used the fact that $\tilde{v}_{i j, k}=\tilde{v}_{i k, j}$ if the emitted parton $i$ is a massless parton, as in the case of the eikonal integral.

\section{B Alternative integrated dipole functions}

At the end of Sect. 5.2.3 we have emphasized that the forms of the $J_{i j}^{a}$ endpoint parts of the $x$-distributions depend on the convention for which kinematical invariant $P^{2}$ is kept fixed during the $x$-integration which is performed over a plus distribution $\left[f\left(x, P^{2}\right)\right]_{+}$. This becomes obvious if we substitute the invariant $P^{2}=2 p_{a} \widetilde{p}_{i j}$ used in Sect. 5.2.3 with another invariant such as, for instance,

$$
P^{2}=Q^{2}=\left(\widetilde{p}_{i j}-x p_{a}\right)^{2}=m_{i j}^{2}-2 x p_{a} \widetilde{p}_{i j},
$$

which was used in Ref. [25]. In this appendix we provide the necessary formulae for this particular variant of treating the integrated dipole functions.

The transition to the parametrization in terms of the new $P^{2}=Q^{2}$ requires some changes in the separation of the one-particle phase space described in Sect. 5.2.1. The rescaled parton masses have to be normalized to $P^{2}$, so we define

$$
\bar{\mu}_{n}^{2}=\frac{m_{n}^{2}}{-Q^{2}}, \quad n=i, j, i j
$$

The previously defined rescaled masses $\mu_{n}$ are, thus, replaced by $\bar{\mu}_{n}$ using

$$
\mu_{n}^{2}=\frac{x \bar{\mu}_{n}^{2}}{1+\bar{\mu}_{i j}^{2}}
$$

in Eqs. (5.48) and (5.49), leading to

$$
\begin{aligned}
& \int\left[\mathrm{d} p_{i}\left(Q ; p_{a}, x\right)\right]=\frac{1}{4}(2 \pi)^{-3+2 \epsilon}\left(-Q^{2}\right)^{1-\epsilon}\left(1+\bar{\mu}_{i j}^{2}\right) \int_{0}^{\bar{x}_{+}} \mathrm{d} x \delta\left(x-x_{i j, a}\right) x^{-1+\epsilon}\left(1-x+\bar{\mu}_{i j}^{2}\right)^{-\epsilon} \\
& \times \int \mathrm{d}^{d-3} \Omega \int_{\bar{z}_{-}(x)}^{\bar{z}_{+}(x)} \mathrm{d} \tilde{z}_{i}\left[\bar{z}_{+}(x)-\tilde{z}_{i}\right]^{-\epsilon}\left[\tilde{z}_{i}-\bar{z}_{-}(x)\right]^{-\epsilon}, \\
& \bar{z}_{ \pm}(x)=\frac{1+\bar{\mu}_{i j}^{2}-x+x \bar{\mu}_{i}^{2}-x \bar{\mu}_{j}^{2} \pm \sqrt{\left(1+\bar{\mu}_{i j}^{2}-x-x \bar{\mu}_{i}^{2}-x \bar{\mu}_{j}^{2}\right)^{2}-4 \bar{\mu}_{i}^{2} \bar{\mu}_{j}^{2} x^{2}}}{2\left(1+\bar{\mu}_{i j}^{2}-x\right)} .
\end{aligned}
$$

From these expressions the upper limit $\bar{x}_{+}$for $x_{i j, a}$ is derived in terms of $\bar{\mu}_{n}$,

$$
\bar{x}_{+}=\frac{1+\bar{\mu}_{i j}^{2}}{1+\left(\bar{\mu}_{i}+\bar{\mu}_{j}\right)^{2}} .
$$

The dipole functions $\mathbf{V}_{i j}^{a}$, defined in Sect. 5.2.2, remain unchanged. The integrated dipole functions

$$
\int\left[\mathrm{d} p_{i}\left(Q ; p_{a}, x\right)\right] \frac{1}{\left(p_{i}+p_{j}\right)^{2}-m_{i j}^{2}}\left\langle\mathbf{V}_{i j}^{a}\right\rangle \equiv \frac{\alpha_{\mathrm{s}}}{2 \pi} \frac{1}{\Gamma(1-\epsilon)}\left(\frac{4 \pi \mu^{2}}{m_{i j}^{2}-Q^{2}}\right)^{\epsilon} \bar{I}_{i j}^{a}(x ; \epsilon)
$$


are given in terms of new auxiliary functions $\bar{I}_{i j}^{a}(x ; \epsilon)$ and their calculation proceeds along the same lines as described in Sect. 5.2.3. The endpoints are split off according to

$$
\begin{aligned}
\bar{I}_{g Q}^{a}(x ; \epsilon) & =C_{\mathrm{F}}\left(\left[\bar{J}_{g Q}^{a}\left(x, \bar{\mu}_{Q}\right)\right]_{+}+\delta(1-x)\left[\bar{J}_{g Q}^{a ; \mathrm{S}}\left(\bar{\mu}_{Q} ; \epsilon\right)+\bar{J}_{g Q}^{a ; \mathrm{NS}}\left(\bar{\mu}_{Q}\right)\right]\right)+\mathrm{O}(\epsilon), \\
\bar{I}_{Q \bar{Q}}^{a}(x ; \epsilon) & =T_{\mathrm{R}}\left(\left[\bar{J}_{Q \bar{Q}}^{a}\left(x, \bar{\mu}_{Q}\right)\right]_{\bar{x}_{+}}+\delta\left(\bar{x}_{+}-x\right)\left[\bar{J}_{Q \bar{Q}}^{a ; S}\left(\bar{\mu}_{Q} ; \epsilon\right)+\bar{J}_{Q \bar{Q}}^{a ; \mathrm{NS}}\left(\bar{\mu}_{Q}\right)\right]\right)+\mathrm{O}(\epsilon),
\end{aligned}
$$

where $\bar{x}_{+}=1 /\left(1+4 \bar{\mu}_{Q}^{2}\right)$. The continuum part for the $Q \rightarrow g Q$ splitting,

$$
\begin{aligned}
{\left[\bar{J}_{g Q}^{a}\left(x, \bar{\mu}_{Q}\right)\right]_{+}=} & \left(\frac{2}{1-x}\left[\ln \left(\frac{1+\bar{\mu}_{Q}^{2}}{1+\bar{\mu}_{Q}^{2}-x}\right)-1\right]+\frac{\left(1+\bar{\mu}_{Q}^{2}\right)^{2}(1-x)}{2\left(1+\bar{\mu}_{Q}^{2}-x\right)^{2}}\right)_{+} \\
& +\left(\frac{2}{1-x}\right)_{+} \ln \left(\frac{2+2 \bar{\mu}_{Q}^{2}-x}{1+\bar{\mu}_{Q}^{2}}\right),
\end{aligned}
$$

and the singular part of the corresponding endpoint contribution,

$$
\begin{aligned}
\bar{J}_{g Q}^{a ; \mathrm{S}}\left(\bar{\mu}_{Q} ; \epsilon\right)= & \frac{1}{\epsilon^{2}}-\frac{\pi^{2}}{3}-\left(\frac{\bar{\mu}_{Q}^{2}}{1+\bar{\mu}_{Q}^{2}}\right)^{-\epsilon}\left(\frac{1}{\epsilon^{2}}+\frac{1}{2 \epsilon}+\frac{\pi^{2}}{6}+2\right) \\
& -\frac{1}{\epsilon} \ln \left(\frac{1+2 \bar{\mu}_{Q}^{2}}{1+\bar{\mu}_{Q}^{2}}\right)+\frac{1}{C_{\mathrm{F}}}\left[\frac{1}{\epsilon} \gamma_{q}+K_{q}\right],
\end{aligned}
$$

result from their counterparts $\left[J_{g Q}^{a}\left(x, \mu_{Q}\right)\right]_{+}$and $J_{g Q}^{a ; \mathrm{S}}\left(\mu_{Q} ; \epsilon\right)$, given in Eqs. (5.58) and (5.59), upon the simple substitutions $\mu_{Q}^{2} \rightarrow x \bar{\mu}_{Q}^{2} /\left(1+\bar{\mu}_{Q}^{2}\right)$ and $\mu_{Q}^{2} \rightarrow \bar{\mu}_{Q}^{2} /\left(1+\bar{\mu}_{Q}^{2}\right)$, respectively. The non-singular endpoint contribution is given by

$$
\begin{aligned}
\bar{J}_{g Q}^{a ; \mathrm{NS}}\left(\bar{\mu}_{Q}\right) & =\frac{2 \pi^{2}}{3}-2 \operatorname{Li}_{2}\left(\frac{1}{1+\bar{\mu}_{Q}^{2}}\right)-2 \operatorname{Li}_{2}\left(\frac{1+\bar{\mu}_{Q}^{2}}{1+2 \bar{\mu}_{Q}^{2}}\right)-\frac{1}{2} \ln ^{2}\left(\frac{1+\bar{\mu}_{Q}^{2}}{1+2 \bar{\mu}_{Q}^{2}}\right) \\
& +\frac{1}{2} \bar{\mu}_{Q}^{2}\left(2+\bar{\mu}_{Q}^{2}\right) \ln \left(\frac{1+\bar{\mu}_{Q}^{2}}{\bar{\mu}_{Q}^{2}}\right)-\frac{1}{2} \bar{\mu}_{Q}^{2} .
\end{aligned}
$$

Note that this does not follow from $J_{g Q}^{a ; \mathrm{NS}}\left(\mu_{Q}\right)$, given in Eq. (5.60), upon substituting $\mu_{Q}$, but a finite difference remains

$$
\begin{aligned}
\Delta \bar{J}_{g Q}^{a ; \mathrm{NS}}\left(\bar{\mu}_{Q}\right) & =\bar{J}_{g Q}^{a ; \mathrm{NS}}\left(\bar{\mu}_{Q}\right)-J_{g Q}^{a ; \mathrm{NS}}\left(\frac{\bar{\mu}_{Q}}{\sqrt{1+\bar{\mu}_{Q}^{2}}}\right) \\
& =\frac{\pi^{2}}{3}-2 \operatorname{Li}_{2}\left(\frac{1}{1+\bar{\mu}_{Q}^{2}}\right)+2 \operatorname{Li}_{2}\left(\frac{-\bar{\mu}_{Q}^{2}}{1+\bar{\mu}_{Q}^{2}}\right)+\frac{1}{2} \ln \left(\frac{1+\bar{\mu}_{Q}^{2}}{1+2 \bar{\mu}_{Q}^{2}}\right) \\
& +\frac{1}{2} \bar{\mu}_{Q}^{2}\left(2+\bar{\mu}_{Q}^{2}\right) \ln \left(\frac{1+\bar{\mu}_{Q}^{2}}{\bar{\mu}_{Q}^{2}}\right)-\frac{\bar{\mu}_{Q}^{2}\left(1+\bar{\mu}_{Q}^{2}\right)}{1+2 \bar{\mu}_{Q}^{2}} .
\end{aligned}
$$

For the $g \rightarrow Q \bar{Q}$ splitting the same features are observed. The continuum part,

$$
\left[\bar{J}_{Q \bar{Q}}^{a}\left(x, \bar{\mu}_{Q}\right)\right]_{\bar{x}_{+}}=\frac{2}{3}\left(\frac{1-x+2 x \bar{\mu}_{Q}^{2}}{(1-x)^{2}} \sqrt{1-\frac{4 x \bar{\mu}_{Q}^{2}}{1-x}}\right)_{\bar{x}_{+}},
$$


results from $\left[J_{Q \bar{Q}}^{a}\left(x, \mu_{Q}\right)\right]_{x_{+}}$, given in Eq. (5.62), upon substituting $\mu_{Q}^{2} \rightarrow x \bar{\mu}_{Q}^{2}$ and $x_{+} \rightarrow \bar{x}_{+}$. The singular endpoint part,

$$
\bar{J}_{Q \bar{Q}}^{a ; \mathrm{S}}\left(\bar{\mu}_{Q} ; \epsilon\right)=-\frac{2}{3 \epsilon}\left[1-\left(\frac{\bar{\mu}_{Q}^{2}}{1+4 \bar{\mu}_{Q}^{2}}\right)^{-\epsilon}\right]-\frac{10}{9},
$$

is obtained from $J_{Q \bar{Q}}^{a ; \mathrm{S}}\left(\mu_{Q} ; \epsilon\right)$, given in Eq. (5.63), by the replacement $\mu_{Q}^{2} \rightarrow \bar{x}_{+} \bar{\mu}_{Q}^{2}$. However, the non-singular endpoint part differs from $J_{Q \bar{Q}}^{a ; \mathrm{NS}}\left(\bar{\mu}_{Q}\right)$, given in Eq. (5.64), in a non-trivial way:

$$
\bar{J}_{Q \bar{Q}}^{a ; \mathrm{NS}}\left(\bar{\mu}_{Q}\right)=\frac{8}{3} \bar{\mu}_{Q}^{2}+\frac{2}{3} \ln \left(\frac{\bar{\mu}_{Q}^{2}}{1+4 \bar{\mu}_{Q}^{2}}\right)+\frac{4}{3}\left(1-2 \bar{\mu}_{Q}^{2}\right) \sqrt{1+4 \bar{\mu}_{Q}^{2}} \ln \left(\frac{\sqrt{1+4 \bar{\mu}_{Q}^{2}}+1}{2 \bar{\mu}_{Q}}\right) .
$$

The difference between $\bar{J}_{Q \bar{Q}}^{a ; \mathrm{NS}}\left(\bar{\mu}_{Q}\right)$ and the reparametrized $J_{Q \bar{Q}}^{a ; \mathrm{NS}}\left(\bar{\mu}_{Q}\right)$ reads

$$
\begin{aligned}
\Delta \bar{J}_{Q \bar{Q}}^{a ; \mathrm{NS}}\left(\bar{\mu}_{Q}\right) & =\bar{J}_{Q \bar{Q}}^{a ; \mathrm{NS}}\left(\bar{\mu}_{Q}\right)-J_{Q \bar{Q}}^{a ; \mathrm{NS}}\left(\frac{\bar{\mu}_{Q}}{\sqrt{1+4 \bar{\mu}_{Q}^{2}}}\right) \\
& =\frac{8}{3} \bar{\mu}_{Q}^{2}-\frac{10}{9}+\left(\frac{10}{9}+\frac{16}{3} \bar{\mu}_{Q}^{2}\right)\left(1+4 \bar{\mu}_{Q}^{2}\right)^{-3 / 2} \\
& +\frac{4}{3}\left[\left(1-2 \bar{\mu}_{Q}^{2}\right) \sqrt{1+4 \bar{\mu}_{Q}^{2}}-1\right] \ln \left(\frac{\sqrt{1+4 \bar{\mu}_{Q}^{2}}+1}{2 \bar{\mu}_{Q}}\right) .
\end{aligned}
$$

Since the non-trivial differences between the two endpoint parametrizations appear only in the integrated dipole functions for final-state emitter and initial-state spectator, the necessary changes in Sects. 6.3 and 6.4 can be easily inferred. The new functions $\overline{\mathcal{K}}_{j}^{a b}$ follow from their previously defined counterparts $\mathcal{K}_{j}^{a b}$ by simple substitutions and adding the extra terms $\Delta \bar{J}_{g Q}^{a ; \mathrm{NS}}$ and $\Delta \bar{J}_{Q \bar{Q}}^{a ; \mathrm{NS}}$,

$$
\begin{aligned}
\overline{\mathcal{K}}_{q}^{a b}\left(x ; Q_{j a}^{2}, m_{j}\right)= & \left.\mathcal{K}_{q}^{a b}\left(x ; s_{j a}, m_{j}\right)\right|_{s_{j a} \rightarrow\left(m_{j}^{2}-Q_{j a}^{2}\right) / x} \\
& +\delta^{a b} \delta(1-x) \Delta \bar{J}_{g Q}^{a ; \mathrm{NS}}\left(\frac{m_{j}}{\sqrt{-Q_{j a}^{2}}}\right), \\
\overline{\mathcal{K}}_{g}^{a b}\left(x ; Q_{j a}^{2}, 0,\left\{m_{F}\right\}\right)= & \left.\mathcal{K}_{g}^{a b}\left(x ; s_{j a}, 0,\left\{m_{F}\right\}\right)\right|_{s_{j a} \rightarrow-Q_{j a}^{2} / x, x_{+} \rightarrow \bar{x}_{+}} \\
& +\delta^{a b} \delta\left(\bar{x}_{+}-x\right) \frac{T_{\mathrm{R}}}{C_{\mathrm{A}}} \sum_{F=1}^{N_{F}} \Delta \bar{J}_{Q \bar{Q}}^{a ; \mathrm{NS}}\left(\frac{m_{F}}{\sqrt{-Q_{j a}^{2}}}\right),
\end{aligned}
$$

where $Q_{j a}^{2}=m_{j}^{2}-x s_{j a}$, not to be confused with $Q_{j a}^{2}=s_{j a}+m_{j}^{2}$ used in Sect. 6.3 of the main text. Note that in the scheme considered in this appendix, all masses of quark are accessible at all values of $Q_{j a}^{2}$, so $N_{F}$ appears in Eq. (B.18) rather than $N_{F}^{j a}$. In fact all occurrences of $N_{F}^{j a}$ in $\boldsymbol{I}$ and $\boldsymbol{K}$ should be replaced by $N_{F}$ in this scheme. 


\section{Dipole functions in SUSY QCD}

In this appendix we define the dipole functions $\mathcal{D}_{i j, k}$ and $\mathcal{D}_{i j}^{a}$ needed to construct the dipole subtraction function specified in Eq. (5.1) for SUSY QCD calculations (see for example Refs. [48, 49, 50, 51]). The new dipoles fall into two classes: one that involves gluinos and gluons and the other that involves squarks and gluons. We consider only those dipoles that lead to $1 / \epsilon$ poles after integration in $d=4-2 \epsilon$ dimensions. Owing to the large masses of the SUSY particles in typical supersymmetric extensions of the SM, the massless limit is not too relevant even at LHC or NLC energies. Thus we do not define dipole functions corresponding to either $g \rightarrow \tilde{g} \tilde{g}, \tilde{q} \tilde{q}$ or splittings involving a squark, a gluino and a quark.

In principle, to calculate one-loop amplitudes in supersymmetric theories one has to use a regularization prescription that respects the supersymmetric Ward identities, for instance, dimensional reduction (DR). Within the dipole formalism, the use of different regularization prescriptions only affects the actual calculation of the cross section contribution $\sigma^{\mathrm{NLO}\{m\}}$ in Eq. (6.8). In this paper we derived our formulae using conventional dimensional regularization (CDR), therefore, the one-loop amplitudes $\left|\mathcal{M}_{m, a b}\left(\left\{p_{i}, m_{i}\right\} ; p_{a}, p_{b}\right)\right|_{(1-\mathrm{loop})}^{2}$ are also needed in CDR. To derive the insertion operator $\boldsymbol{I}(\epsilon)$ for SUSY QCD one has two options, either to use DR throughout and the transition rules between CDR and DR elucidated in Refs. [44, 11, 31, 45], or to use CDR throughout and correct for the violation of the SUSY Ward identities in the calculation of the one-loop contribution $\left|\mathcal{M}_{m, a b}\left(\left\{p_{i}, m_{i}\right\} ; p_{a}, p_{b}\right)\right|_{(1-\text { loop })}^{2}$ as explained in Refs. [52, 48]. We choose to do the latter.

As discussed in Sect. 2, we do not consider massive partons in the initial state, therefore,

new dipoles of the $\mathcal{D}_{j}^{a i}$ type do not appear in the supersymmetric theory. The dipole functions that involve gluinos are obtained immediately by changing the colour factor $C_{\mathrm{F}}$ in Eqs. (5.16) and (5.50) to $C_{\mathrm{A}}$. As a result the integrated dipole functions are also obtained by this simple change in Eqs. (5.23) and (5.56).

The flavour and the spin of the spectators do not influence the dipole functions, therefore, we have to consider only final-state emitter squarks as new cases. Thus, we define the splitting functions $\mathbf{V}_{i j, k}$ and $\mathbf{V}_{i j}^{a}$ for the $\tilde{q} \rightarrow g \tilde{q}$ splitting. The case $\overline{\tilde{q}} \rightarrow g \overline{\tilde{q}}$ is formally identical to $\tilde{q} \rightarrow g \tilde{q}$.

When the spectator is also in the final state, then we define

$$
\begin{aligned}
\left\langle s\left|\mathbf{V}_{g \tilde{q}, k}\right| s^{\prime}\right\rangle & =8 \pi \mu^{2 \epsilon} \alpha_{\mathrm{s}} C_{\mathrm{F}}\left\{\frac{2}{1-\tilde{z}_{j}\left(1-y_{i j, k}\right)}-\frac{\tilde{v}_{i j, k}}{v_{i j, k}}\left(2+\frac{m_{\tilde{q}}^{2}}{p_{i} p_{j}}\right)\right\} \delta_{s s^{\prime}} \\
& =\left\langle\mathbf{V}_{g \tilde{q}, k}\right\rangle \delta_{s s^{\prime}} .
\end{aligned}
$$

We write the integral of $\left\langle\mathbf{V}_{g \tilde{q}, k}\right\rangle /\left(2 p_{g} p_{\tilde{q}}\right)$ over the one-parton subspace in Eq. (5.11) in the form of Eqs. (5.22) and (5.23). The eikonal integral depends only on the mass of the emitter, but not on its flavour or spin. The collinear integral is obtained as

$$
I_{g \tilde{q}, k}^{\mathrm{coll}}\left(\mu_{\tilde{q}}, \mu_{k}\right)=\frac{2}{\epsilon}-\frac{\mu_{\tilde{q}}^{-2 \epsilon}}{\epsilon}-2 \mu_{\tilde{q}}^{-2 \epsilon}+6-2 \ln \left[\left(1-\mu_{k}\right)^{2}-\mu_{\tilde{q}}^{2}\right]+\frac{4 \mu_{k}\left(\mu_{k}-1\right)}{1-\mu_{\tilde{q}}^{2}-\mu_{k}^{2}}+\mathrm{O}(\epsilon) .
$$


If the spectator is in the initial state, we define

$$
\begin{aligned}
\left\langle s\left|\mathbf{V}_{g \tilde{q}}^{a}\right| s^{\prime}\right\rangle & =8 \pi \mu^{2 \epsilon} \alpha_{\mathrm{s}} C_{\mathrm{F}}\left\{\frac{2}{2-x_{i j, a}-\tilde{z}_{j}}-2-\frac{m_{\tilde{q}}^{2}}{p_{i} p_{j}}\right\} \delta_{s s^{\prime}} \\
& =\left\langle\mathbf{V}_{g \tilde{q}}^{a}\right\rangle \delta_{s s^{\prime}} .
\end{aligned}
$$

We write the integral of $\left\langle\mathbf{V}_{g \tilde{q}}^{a}\right\rangle /\left(2 p_{g} p_{\tilde{q}}\right)$ over the one-parton subspace in Eq. (5.48) in the form of Eqs. (5.53) and (5.56). The continuum part is

$$
\left[J_{g \tilde{q}}^{a}\left(x, \mu_{\tilde{q}}\right)\right]_{+}=\left(-\frac{2}{1-x}\left[1+\ln \left(1-x+\mu_{\tilde{q}}^{2}\right)\right]\right)_{+}+\left(\frac{2}{1-x}\right)_{+} \ln \left(2+\mu_{\tilde{q}}^{2}-x\right) .
$$

The endpoint parts are

$$
\begin{gathered}
J_{g \tilde{q}}^{a ; \mathrm{S}}\left(\mu_{\tilde{q}} ; \epsilon\right)=\frac{1}{\epsilon^{2}}-\frac{\pi^{2}}{3}-\mu_{\tilde{q}}^{-2 \epsilon}\left(\frac{1}{\epsilon^{2}}+\frac{1}{\epsilon}+\frac{\pi^{2}}{6}+2\right)-\frac{1}{\epsilon} \ln \left(1+\mu_{\tilde{q}}^{2}\right)+\frac{1}{C_{\mathrm{F}}}\left(\frac{1}{\epsilon} \gamma_{\tilde{q}}+K_{\tilde{q}}\right) \\
J_{g \tilde{q}}^{a ; \mathrm{NS}}\left(\mu_{\tilde{q}}\right)=\frac{\pi^{2}}{3}-2 \operatorname{Li}_{2}\left(\frac{1}{1+\mu_{\tilde{q}}^{2}}\right)-2 \operatorname{Li}_{2}\left(-\mu_{\tilde{q}}^{2}\right)-\frac{1}{2} \ln ^{2}\left(1+\mu_{\tilde{q}}^{2}\right)
\end{gathered}
$$

where

$$
\gamma_{\tilde{q}}=2 C_{\mathrm{F}}, \quad K_{\tilde{q}}=\left(4-\frac{\pi^{2}}{6}\right) C_{\mathrm{F}}
$$

Using these results, we obtain the functions needed for the construction of the insertion operators $\boldsymbol{I}_{m}$ and $\boldsymbol{K}^{a, b}$. The singular functions $\mathcal{V}^{(\mathrm{S})}$ are independent of the flavour and spin. The non-singular function $\mathcal{V}_{j}^{(\mathrm{NS})}$ for the gluino is

$$
\mathcal{V}_{\tilde{g}}^{(\mathrm{NS})}\left(s_{j k}, m_{j}, m_{k}\right)=\mathcal{V}_{q}^{(\mathrm{NS})}\left(s_{j k}, m_{j}, m_{k}\right)
$$

and for the squark it is

$$
\begin{gathered}
\mathcal{V}_{\tilde{q}}^{(\mathrm{NS})}\left(s_{j k}, m_{j}, m_{k}\right)=\frac{\gamma_{\tilde{q}}}{\boldsymbol{T}_{\tilde{q}}^{2}} \ln \frac{s_{j k}}{Q_{j k}^{2}}-2 \ln \frac{\left(Q_{j k}-m_{k}\right)^{2}-m_{j}^{2}}{Q_{j k}^{2}}+\frac{4 m_{k}\left(m_{k}-Q_{j k}\right)}{s_{j k}}+\frac{\pi^{2}}{2} \\
+\frac{1}{v_{j k}}\left[\ln \rho^{2} \ln \left(1+\rho^{2}\right)+2 \operatorname{Li}_{2}\left(\rho^{2}\right)-\mathrm{Li}_{2}\left(1-\rho_{j}^{2}\right)-\mathrm{Li}_{2}\left(1-\rho_{k}^{2}\right)-\frac{\pi^{2}}{6}\right]
\end{gathered}
$$

if $k$ is massive, and

$$
\mathcal{V}_{\tilde{q}}^{(\mathrm{NS})}\left(s_{j k}, m_{j}, 0\right)=\frac{\gamma_{\tilde{q}}}{\boldsymbol{T}_{\tilde{q}}^{2}} \ln \frac{s_{j k}}{Q_{j k}^{2}}+\frac{\pi^{2}}{6}-\mathrm{Li}_{2}\left(\frac{s_{j k}}{Q_{j k}^{2}}\right)-2 \ln \frac{s_{j k}}{Q_{j k}^{2}}
$$

if $k$ is massless. The singular function $\Gamma_{j}$ for the gluino is

$$
\Gamma_{\tilde{g}}\left(\mu, m_{\tilde{g}} ; \epsilon\right)=\boldsymbol{T}_{\tilde{g}}^{2}\left(\frac{1}{\epsilon}-\ln \frac{m_{\tilde{g}}^{2}}{\mu^{2}}-2\right)+\gamma_{\tilde{g}} \ln \frac{m_{\tilde{g}}^{2}}{\mu^{2}}=C_{\mathrm{A}}\left[\frac{1}{\epsilon}+\frac{1}{2} \ln \frac{m_{\tilde{g}}^{2}}{\mu^{2}}-2\right],
$$

and the flavour constants are

$$
\gamma_{\tilde{g}}=\frac{3}{2} C_{\mathrm{A}}, \quad K_{\tilde{g}}=\left(\frac{7}{2}-\frac{\pi^{2}}{6}\right) C_{\mathrm{A}} .
$$


The singular function $\Gamma_{j}$ for the squark is

$$
\Gamma_{\tilde{q}}\left(\mu, m_{\tilde{q}} ; \epsilon\right)=\boldsymbol{T}_{\tilde{q}}^{2}\left(\frac{1}{\epsilon}-\ln \frac{m_{\tilde{q}}^{2}}{\mu^{2}}-2\right)+\gamma_{\tilde{q}} \ln \frac{m_{\tilde{q}}^{2}}{\mu^{2}}=C_{\mathrm{F}}\left[\frac{1}{\epsilon}+\ln \frac{m_{\tilde{q}}^{2}}{\mu^{2}}-2\right] .
$$

Finally, the functions $\mathcal{K}_{j}^{a, b}$ for the gluino are

$$
\mathcal{K}_{\tilde{g}}^{a, b}\left(x ; s_{j a}, m_{j}\right)=\mathcal{K}_{q}^{a, b}\left(x ; s_{j a}, m_{j}\right),
$$

and those for the squark are

$$
\begin{gathered}
\mathcal{K}_{\tilde{q}}^{a, b}\left(x ; s_{j a}, m_{j}\right)=\mathcal{K}_{q}^{a, b}\left(x ; s_{j a}, m_{j}\right)-\delta^{a b}\left(\frac{s_{j a}^{2}(1-x)}{2\left[s_{j a}(1-x)+m_{j}^{2}\right]^{2}}\right)+ \\
+\delta^{a b} \delta(1-x)\left[-\left(\frac{m_{j}^{2}}{s_{j a}} \ln \frac{m_{j}^{2}}{Q_{j a}^{2}}+\frac{m_{j}^{2}}{2 Q_{j a}^{2}}\right)+\frac{\gamma_{q}-\gamma_{\tilde{q}}}{C_{\mathrm{F}}}\right] .
\end{gathered}
$$

\section{Example Applications}

We illustrate our method with the three simplest examples, $e^{+} e^{-}$annihilation to heavy quarks, $e^{+} e^{-} \rightarrow Q \bar{Q}$, to heavy quarks and a jet, $e^{+} e^{-} \rightarrow Q \bar{Q} g$ and heavy quark production in deep inelastic scattering, $e p \rightarrow e Q \bar{Q}+X$.

\section{$\mathrm{D} .1 e^{+} e^{-} \rightarrow Q \bar{Q}$}

We begin by recalling the tree-level result to set the notation. Since our aim is to illustrate our method, we try to keep the analytical formulae simple by taking the average over event plane orientations and neglecting electron polarization. The extension to oriented and polarized observables is straightforward.

In order to match consistently with the one-loop corrections that are available in the literature, it is necessary to evaluate the tree-level matrix element in $d$ dimensions. In fact, since all singularities can be cancelled before averaging over event orientation, it is sufficient to consistently evaluate the hadronic tensor in $d$ dimensions. We are then free to take the rest of the process, as well as the remaining angular integrations, in 4 dimensions. Since after averaging over event orientation and polarization we only encounter Dirac traces with no or two $\gamma^{5}$ matrices, we can easily eliminate $\gamma^{5}$ by taking it to be totally anticommuting in $d$ dimensions and using $\gamma_{5}^{2}=1$.

With this prescription, the $d$-dimensional matrix element is identical to the 4-dimensional one which, labelling the momenta by $e^{+}+e^{-} \rightarrow \gamma^{*} / Z(q) \rightarrow Q\left(p_{1}\right)+\bar{Q}\left(p_{2}\right)$, is given by 53

$$
\left|\mathcal{M}_{2}\right|^{2}=\left(g^{V V}+g^{A A}\right)\left(1+2 \mu_{Q}^{2}\right)-g^{A A}\left(6 \mu_{Q}^{2}\right)
$$


where $\mu_{Q} \equiv m_{Q} / \sqrt{s}$, with $s=q^{2}$, and the normalization is such that the cross section is given by

$$
\sigma^{\mathrm{LO}}=\sigma_{0} v\left|\mathcal{M}_{2}\right|^{2} F_{J}^{(2)}\left(p_{1}, p_{2}\right)
$$

where $v=\sqrt{1-4 \mu_{Q}^{2}}$ is the velocity of the heavy quark in the centre-of-mass frame and $F_{J}$ is our (infrared-safe) observable. The coupling constants and point-like cross section appearing in Eqs. (D.1) and (D.2) are given by

$$
\begin{aligned}
g^{V V} & =Q_{Q}^{2}-2 g_{v}^{e} g_{v}^{Q} Q_{Q} \operatorname{Re}\{\chi(s)\}+\left(g_{v}^{e 2}+g_{a}^{e 2}\right) g_{v}^{Q^{2}}|\chi(s)|^{2} \\
g^{A A} & =\left(g_{v}^{e 2}+g_{a}^{e 2}\right) g_{a}^{Q^{2}}|\chi(s)|^{2} \\
\sigma_{0} & =N_{\mathrm{c}} \frac{4 \pi \alpha^{2}}{3 s}
\end{aligned}
$$

where $g_{v}^{f}=T_{3}^{f}-2 Q_{f} \sin ^{2} \theta_{w}, g_{a}^{f}=T_{3}^{f}, Q_{f}$ is the electric charge of fermion type $f$ and $\theta_{w}$ is the weak mixing angle. The function $\chi(s)$ parametrizes the $\mathrm{Z}^{0}$ propagator and coupling factors,

$$
\chi(s)=\frac{1}{4 \sin ^{2} \theta_{w} \cos ^{2} \theta_{w}} \frac{s}{s-m_{Z}^{2}+i m_{Z} \Gamma_{Z}},
$$

with $m_{Z}$ and $\Gamma_{Z}$ the mass and width of the $\mathrm{Z}^{0}$.

The NLO real emission process is $e^{+} e^{-} \rightarrow \gamma^{*} / Z(q) \rightarrow Q\left(p_{1}\right)+\bar{Q}\left(p_{2}\right)+g\left(p_{3}\right)$, with matrix element $\mathcal{M}_{3}\left(p_{1}, p_{2}, p_{3}\right)$. In discussing the NLO corrections to the $Q \bar{Q} g$ process, we will need to discuss the $d$-dimensional behaviour of $\mathcal{M}_{3}$ in more detail, so we explicitly indicate the number of dimensions in which it is evaluated. In four dimensions we can rewrite the known result [53] as

$$
\begin{aligned}
\left|\mathcal{M}_{3}^{(4)}\left(p_{1}, p_{2}, p_{3}\right)\right|^{2}=C_{\mathrm{F}} \frac{8 \pi \alpha_{\mathrm{s}}}{s} & \\
\times & \left\{\left|\mathcal{M}_{2}\right|^{2}\left[\frac{1}{\left(1-x_{1}\right)}\left(\frac{2\left(1-2 \mu_{Q}^{2}\right)}{2-x_{1}-x_{2}}-2-\frac{2 \mu_{Q}^{2}}{1-x_{1}}\right)+\left(x_{1} \leftrightarrow x_{2}\right)\right]\right. \\
& \left.+\left[g^{V V}+g^{A A}\left(1+2 \mu_{Q}^{2}\right)\right]\left(\frac{1-x_{2}}{1-x_{1}}+\frac{1-x_{1}}{1-x_{2}}\right)+g^{A A} 4 \mu_{Q}^{2}\right\},
\end{aligned}
$$

where $x_{i} \equiv 2 p_{i} \cdot q / q^{2}$. In terms of these variables, the phase space is given by

$$
\mathrm{d} \Phi^{(3)}=\frac{s}{16 \pi^{2}} \mathrm{~d} x_{1} \mathrm{~d} x_{2} \Theta\left(x_{+}-x_{2}\right) \Theta\left(x_{2}-x_{-}\right) \Theta\left(1-x_{1}\right) \Theta\left(x_{1}-2 \mu_{Q}\right),
$$

with

$$
x_{ \pm}=\frac{\left(2-x_{1}\right)\left(1-x_{1}+2 \mu_{Q}^{2}\right) \pm\left(1-x_{1}\right) \sqrt{x_{1}^{2}-4 \mu_{Q}^{2}}}{2\left(1-x_{1}+\mu_{Q}^{2}\right)} .
$$

The three-parton cross section is then

$$
\sigma^{(3)}=\sigma_{0} \int \mathrm{d} \Phi^{(3)}\left|\mathcal{M}_{3}\right|^{2} F_{J}^{(3)}\left(p_{1}, p_{2}, p_{3}\right),
$$

where we used a different normalization from that in Eq. (3.7). 
According to the dipole subtraction method, this integral is rendered finite by subtracting from it an auxiliary cross section constructed from the two dipole contributions, $\mathcal{D}_{31,2}$ and $\mathcal{D}_{32,1}$, defined by Eq. (5.2). The colour and spin algebra are trivial in this case, and we obtain

$$
\mathcal{D}_{31,2}\left(p_{1}, p_{2}, p_{3}\right)=\frac{1}{2 p_{3} \cdot p_{1}}\left\langle\mathbf{V}_{g_{3} Q_{1}, 2}\right\rangle\left|\mathcal{M}_{2}\right|^{2},
$$

with

$$
\left\langle\mathbf{V}_{g_{3} Q_{1}, 2}\right\rangle=8 \pi \alpha_{\mathrm{s}} C_{\mathrm{F}}\left\{\frac{2}{1-\tilde{z}_{1}\left(1-y_{31,2}\right)}-\frac{\tilde{v}_{31,2}}{v_{31,2}}\left[1+\tilde{z}_{1}+\frac{m_{Q}^{2}}{p_{3} \cdot p_{1}}\right]\right\} .
$$

Inserting the definitions of $y_{31,2}, \tilde{z}_{1}, v_{31,2}$ and $\tilde{v}_{31,2}$ from Eqs. (5.12), (5.14) and (5.8), we obtain

$$
\begin{aligned}
& \mathcal{D}_{31,2}\left(p_{1}, p_{2}, p_{3}\right)=C_{\mathrm{F}} \frac{8 \pi \alpha_{\mathrm{s}}}{s}\left|\mathcal{M}_{2}\right|^{2} \\
& \quad \times \frac{1}{1-x_{2}}\left\{\frac{2\left(1-2 \mu_{Q}^{2}\right)}{2-x_{1}-x_{2}}-\sqrt{\frac{1-4 \mu_{Q}^{2}}{x_{2}^{2}-4 \mu_{Q}^{2}}} \frac{x_{2}-2 \mu_{Q}^{2}}{1-2 \mu_{Q}^{2}}\left[2+\frac{x_{1}-1}{x_{2}-2 \mu_{Q}^{2}}+\frac{2 \mu_{Q}^{2}}{1-x_{2}}\right]\right\} .
\end{aligned}
$$

The associated dipole kinematics are given by

$$
\widetilde{p}_{2}^{\mu}=\frac{1}{2} q^{\mu}+\frac{\sqrt{1-4 \mu_{Q}^{2}}}{\sqrt{x_{2}^{2}-4 \mu_{Q}^{2}}}\left(p_{2}^{\mu}-\frac{1}{2} x_{2} q^{\mu}\right), \quad \widetilde{p}_{31}^{\mu}=\frac{1}{2} q^{\mu}-\frac{\sqrt{1-4 \mu_{Q}^{2}}}{\sqrt{x_{2}^{2}-4 \mu_{Q}^{2}}}\left(p_{2}^{\mu}-\frac{1}{2} x_{2} q^{\mu}\right) .
$$

The dipole $\mathcal{D}_{32,1}$ is obtained from $\mathcal{D}_{31,2}$ by the replacement $p_{1} \leftrightarrow p_{2}$.

Combining the expressions for the real and auxiliary cross sections, we obtain the threeparton integral,

$$
\begin{aligned}
& \sigma^{\mathrm{NLO}\{3\}}=\sigma_{0} C_{\mathrm{F}} \frac{\alpha_{\mathrm{s}}}{2 \pi} \int \mathrm{d} x_{1} \mathrm{~d} x_{2} \\
& \times\left\{| \mathcal { M } _ { 2 } | ^ { 2 } \left[\frac{1}{1-x_{1}}\left(\frac{2\left(1-2 \mu_{Q}^{2}\right)}{2-x_{1}-x_{2}}-2-\frac{2 \mu_{Q}^{2}}{1-x_{1}}\right) F_{J}^{(3)}\left(p_{1}, p_{2}, p_{3}\right)\right.\right. \\
& \quad-\frac{1}{1-x_{1}}\left(\frac{2\left(1-2 \mu_{Q}^{2}\right)}{2-x_{1}-x_{2}}-\sqrt{\frac{1-4 \mu_{Q}^{2}}{x_{1}^{2}-4 \mu_{Q}^{2}}} \frac{x_{1}-2 \mu_{Q}^{2}}{1-2 \mu_{Q}^{2}}\left(2+\frac{x_{2}-1}{x_{1}-2 \mu_{Q}^{2}}+\frac{2 \mu_{Q}^{2}}{1-x_{1}}\right)\right) \\
& \left.\quad \times F_{J}^{(2)}\left(\widetilde{p}_{1}, \widetilde{p}_{32}\right)+\left(x_{1} \leftrightarrow x_{2}, \widetilde{p}_{1} \rightarrow \widetilde{p}_{31}, \widetilde{p}_{32} \rightarrow \widetilde{p}_{2}\right)\right] \\
& \left.+\left[\left(g^{V V}+g^{A A}\left(1+2 \mu_{Q}^{2}\right)\right)\left(\frac{1-x_{2}}{1-x_{1}}+\frac{1-x_{1}}{1-x_{2}}\right)+g^{A A} 4 \mu_{Q}^{2}\right] F_{J}^{(3)}\left(p_{1}, p_{2}, p_{3}\right)\right\} .(\mathrm{D} .15)
\end{aligned}
$$

It is straightforward to check that the soft singularity cancels between the different terms in Eq. (D.15), provided the observable is infrared safe (implying that $F_{J}^{(3)} \rightarrow F_{J}^{(2)}$ in the soft limit). Furthermore, for any quasi-collinear-safe observable (implying also that $F_{J}^{(3)} \rightarrow F_{J}^{(2)}$ in the quasi-collinear limit), Eq. (D.15) is finite in the small-mass limit. In fact, taking the quark mass to zero, one exactly recovers the massless result in Eq. (D.7) of Ref. [11].

Next we have to evaluate the insertion operator $\boldsymbol{I}(\epsilon)$, which gives the integral of the auxiliary cross section and combine it with the virtual cross section. The one-loop matrix 
element was calculated in Ref. [54] and is given byث*,

$$
\begin{aligned}
\left|\mathcal{M}_{2}\right|_{(1-\text { loop })}^{2}= & \left(\frac{4 \pi \mu^{2}}{m_{Q}^{2}}\right)^{\epsilon} \frac{1}{\Gamma(1-\epsilon)} 2 \operatorname{Re}\left\{f_{1}\right\}\left|\mathcal{M}_{2}\right|^{2} \\
& +2 \operatorname{Re}\left\{f_{2}\right\}\left(\frac{3}{2}\left(g^{V V}+g^{A A}\right)-\left(\frac{5}{2}-4 \mu_{Q}^{2}\right) g^{A A}\right) .
\end{aligned}
$$

The form factors appearing in Eq. (D.16) are given by

$$
\begin{aligned}
\operatorname{Re}\left\{f_{1}\right\}= & C_{\mathrm{F}} \frac{\alpha_{\mathrm{s}}}{2 \pi}\left\{\frac{-1}{\epsilon}\left(1+\frac{1+v^{2}}{2 v} \ln \frac{1-v}{1+v}\right)-2-\frac{1+2 v^{2}}{2 v} \ln \frac{1-v}{1+v}\right. \\
& \left.+\frac{1+v^{2}}{v}\left[\operatorname{Li}_{2}\left(\frac{1-v}{1+v}\right)+\frac{\pi^{2}}{3}-\frac{1}{4} \ln ^{2} \frac{1-v}{1+v}+\ln \frac{1-v}{1+v} \ln \frac{2 v}{1+v}\right]\right\}, \\
\operatorname{Re}\left\{f_{2}\right\}= & C_{\mathrm{F}} \frac{\alpha_{\mathrm{s}}}{2 \pi} \frac{1-v^{2}}{2 v} \ln \frac{1-v}{1+v} .
\end{aligned}
$$

For later convenience, we define a function $f_{f}$,

$$
\operatorname{Re}\left\{f_{1}\right\}=C_{\mathrm{F}} \frac{\alpha_{\mathrm{s}}}{2 \pi}\left\{\frac{-1}{\epsilon}\left(1+\frac{1+v^{2}}{2 v} \ln \frac{1-v}{1+v}\right)-2-\frac{3}{2} \ln \frac{1-v}{1+v}+\frac{2 \pi^{2}}{3}-\frac{1}{2} \ln ^{2} \frac{1-v}{1+v}+f_{f}(v)\right\},
$$

such that $f_{f}(v)$ vanishes in the small-mass (i.e. $v \rightarrow 1$ ) limit.

The general expression for $\boldsymbol{I}(\epsilon)$ is given in Eq. (6.16). In our case, the colour structure is trivial and we obtain

$$
\begin{aligned}
& { }_{2}\left\langle 1,2\left|\boldsymbol{I}_{2}\left(\epsilon, \mu^{2} ;\left\{p_{i}, m_{i}\right\}\right)\right| 1,2\right\rangle_{2}=\left|\mathcal{M}_{2}\right|^{2} \times 2 C_{\mathrm{F}} \frac{\alpha_{\mathrm{s}}}{2 \pi} \frac{(4 \pi)^{\epsilon}}{\Gamma(1-\epsilon)} \\
& \quad \times\left[\left(\frac{\mu^{2}}{s_{12}}\right)^{\epsilon}\left(\mathcal{V}_{q}\left(s_{12}, m_{Q}, m_{Q} ; \epsilon\right)-\frac{\pi^{2}}{3}\right)+\frac{1}{C_{\mathrm{F}}} \Gamma_{q}\left(\mu, m_{Q} ; \epsilon\right)+\frac{3}{2} \ln \frac{\mu^{2}}{s_{12}}+5-\frac{\pi^{2}}{6}\right],
\end{aligned}
$$

where $s_{12}=2 p_{1} \cdot p_{2}=s-2 m_{Q}^{2}$ and we have suppressed the dependence on $\left\{m_{F}\right\}$ and $\kappa$, which do not enter our cross section. The function $\mathcal{V}_{q}$ is decomposed into singular and non-singular parts according to Eq. (6.18). The $\epsilon$-expansion of the singular term is given in Eq. (6.20). In our case it is

$$
\mathcal{V}^{(\mathrm{S})}\left(s_{12}, m_{Q}, m_{Q} ; \epsilon\right)=\frac{1+v^{2}}{2 v}\left[\frac{1}{\epsilon} \ln \frac{1-v}{1+v}-\frac{1}{2} \ln ^{2} \frac{1-v}{1+v}-\frac{\pi^{2}}{6}+\ln \frac{1-v}{1+v} \ln \frac{2}{1+v^{2}}\right] .
$$

The non-singular term is given in Eq. (6.21), and is given by

$$
\begin{aligned}
& \mathcal{V}_{q}^{(\mathrm{NS})}\left(s_{12}, m_{Q}, m_{Q}\right)=\frac{3}{2} \ln \frac{1+v^{2}}{2} \\
& +\frac{1+v^{2}}{2 v}\left[2 \ln \frac{1-v}{1+v} \ln \frac{2\left(1+v^{2}\right)}{(1+v)^{2}}+2 \operatorname{Li}_{2}\left(\left(\frac{1-v}{1+v}\right)^{2}\right)-2 \operatorname{Li}_{2}\left(\frac{2 v}{1+v}\right)-\frac{\pi^{2}}{6}\right] \\
& +\ln \left(1-\frac{1}{2} \sqrt{1-v^{2}}\right)-2 \ln \left(1-\sqrt{1-v^{2}}\right)-\frac{1-v^{2}}{1+v^{2}} \ln \frac{\sqrt{1-v^{2}}}{2-\sqrt{1-v^{2}}} \\
& \quad-\frac{\sqrt{1-v^{2}}}{2-\sqrt{1-v^{2}}}+2 \frac{1-v^{2}-\sqrt{1-v^{2}}}{1+v^{2}}+\frac{\pi^{2}}{2} .
\end{aligned}
$$

** Note that we have inserted a factor of $\left(4 \pi \mu^{2} / m_{Q}^{2}\right)^{\epsilon} / \Gamma(1-\epsilon)$, where $\mu$ is the dimensional-regularization scale, which is necessary for consistency with our notation. 
Note that in the massless limit, $\mathcal{V}_{q}^{(\mathrm{NS})}$ vanishes. The function $\Gamma_{q}$ appearing in Eq. (D.20) is simply given by

$$
\Gamma_{q}\left(\mu, m_{Q} ; \epsilon\right)=C_{\mathrm{F}}\left[\frac{1}{\epsilon}+\frac{1}{2} \ln \frac{m_{Q}^{2}}{\mu^{2}}-2\right] \text {. }
$$

Combining the virtual and auxiliary cross sections, we obtain a two-parton cross section that is finite as $\epsilon \rightarrow 0$. Setting $\epsilon=0$, we obtain (recall that the leading order cross section, $\sigma^{\mathrm{LO}}$, is defined by the observable $\left.F_{J}^{(2)}\left(p_{1}, p_{2}\right)\right)$,

$$
\begin{aligned}
\sigma^{\mathrm{NLO}\{2\}=} & \sigma^{\mathrm{LO}} 2 C_{\mathrm{F}} \frac{\alpha_{\mathrm{s}}}{2 \pi}\left\{1-\frac{(1-v)^{2}}{v} \frac{\pi^{2}}{12}-\frac{3}{2} \ln \frac{2\left(1+v^{2}\right)}{(1+v)^{2}}\right. \\
& \left.+\ln \frac{1-v}{1+v}\left(\frac{(1-v)^{2}}{4 v} \ln \frac{1-v}{1+v}+\frac{1+v^{2}}{v} \ln \frac{1+v}{1+v^{2}}\right)+f_{f}(v)+\mathcal{V}_{q}^{(\mathrm{NS})}\right\} \\
& +2 \operatorname{Re}\left\{f_{2}\right\} v \sigma_{0}\left(\left(g^{V V}+g^{A A}\right)\left(\frac{3}{2}\right)-g^{A A}\left(\frac{5}{2}-4 \mu_{Q}^{2}\right)\right) F_{J}^{(2)}\left(p_{1}, p_{2}\right) .
\end{aligned}
$$

Note that not only have all singularities cancelled, but also all terms that are singular in the small-mass limit. Furthermore, in this small-mass limit $\sigma^{\mathrm{NLO}\{2\}}$ agrees with the exactly massless prediction given in Eq. (D.11) of Ref. [11],

$$
\lim _{v \rightarrow 1} \sigma^{\mathrm{NLO}\{2\}}=\sigma^{\mathrm{LO}} C_{\mathrm{F}} \frac{\alpha_{\mathrm{s}}}{\pi} .
$$

As a check of our results, we can use them to obtain the total cross section, by setting $F_{J}^{(3)}=F_{J}^{(2)}=1$. The integral in Eq. (D.15) can be performed analytically, but the result does not have a compact form, so we give its expansion in powers of $\mu_{Q}$,

$$
\begin{aligned}
\sigma^{\mathrm{NLO}\{3\}=} & \sigma_{0} C_{\mathrm{F}} \frac{\alpha_{\mathrm{s}}}{2 \pi}\left\{\left(g^{V V}+g^{A A}\right)\left[-\frac{1}{2}+\mu_{Q}^{2}\left(-2 \ln \mu_{Q}^{2}+3\right)+4 \mu_{Q}^{3}+\mathrm{O}\left(\mu_{Q}^{4} \ln \mu_{Q}^{2}\right)\right]\right. \\
& \left.+g^{A A}\left[\mu_{Q}^{2}\left(-8 \ln \mu_{Q}^{2}-15\right)+\mathrm{O}\left(\mu_{Q}^{4} \ln \mu_{Q}^{2}\right)\right]\right\}
\end{aligned}
$$

It is worth noting that no terms linear in $\mu_{Q}$ arise. Although there are no such terms in the real or virtual cross sections, they could in principle arise in the auxiliary cross section, cancelling between the two- and three-parton cross sections. If this did happen it would worsen the numerical convergence in the limit of small but finite masses. Such terms do appear for all higher odd-integer powers of $\mu_{Q}$, but are not problematic.

Combining with $\sigma^{\mathrm{NLO}\{2\}}$ from Eq. (D.24), we obtain

$$
\begin{aligned}
\sigma^{\mathrm{NLO}}= & \sigma_{0} C_{\mathrm{F}} \frac{\alpha_{\mathrm{s}}}{2 \pi}\left\{\left(g^{V V}+g^{A A}\right)\left[\frac{3}{2}+18 \mu_{Q}^{2}+\mathrm{O}\left(\mu_{Q}^{4} \ln \mu_{Q}^{2}\right)\right]\right. \\
& \left.+g^{A A}\left[\mu_{Q}^{2}\left(-18 \ln \mu_{Q}^{2}-27\right)+\mathrm{O}\left(\mu_{Q}^{4} \ln \mu_{Q}^{2}\right)\right]\right\},
\end{aligned}
$$

in agreement with the result given in, for example, Ref. [55]. In fact, expanding the two results to arbitrary order in $\mu_{Q}$ we obtain perfect agreement. 
The example application discussed in this appendix has also been considered in Ref. 56 within the context of the methods of Refs. [7] and [30]. As mentioned in Sect. 1, the formalisms of Refs. [7, 30] are not aimed at smoothly controlling the small-mass limit. This is explicitly shown, for instance, by comparing our Eq. (D.24) with the analogous equations (the sum of Eqs. (15) and (16) for the phase-space slicing method of Ref. [7]; the sum of Eqs. (16) and (24) for the dipole method of Ref. [30]) in Ref. [56], which are singular in the small-mass limit.

\section{$\mathrm{D} .2 e^{+} e^{-} \rightarrow Q \bar{Q} g$}

We continue to average over event orientation and polarization. As in the $e^{+} e^{-} \rightarrow Q \bar{Q}$ case, we require the tree level matrix element evaluated in $d$ dimensions, which is given by

$$
\begin{aligned}
\left|\mathcal{M}_{3}^{(d)}\left(p_{1}, p_{2}, p_{3}\right)\right|^{2} & =\left|\mathcal{M}_{3}^{(4)}\left(p_{1}, p_{2}, p_{3}\right)\right|^{2}-\epsilon\left|\mathcal{M}_{3}^{(\prime)}\left(p_{1}, p_{2}, p_{3}\right)\right|^{2} \\
\left|\mathcal{M}_{3}^{(\prime)}\left(p_{1}, p_{2}, p_{3}\right)\right|^{2} & =C_{\mathrm{F}} \frac{8 \pi \alpha_{\mathrm{s}}}{s}\left\{\left(g^{V V}+g^{A A}\right)+g^{A A}\left(2 \mu_{Q}^{2}\right)\right\} \frac{\left(2-x_{1}-x_{2}\right)^{2}}{\left(1-x_{1}\right)\left(1-x_{2}\right)}
\end{aligned}
$$

At NLO, three different real-emission processes contribute: (a) $e^{+} e^{-} \rightarrow Q\left(p_{1}\right)+\bar{Q}\left(p_{2}\right)+$ $g\left(p_{3}\right)+g\left(p_{4}\right) ;(\mathrm{b}) e^{+} e^{-} \rightarrow Q\left(p_{1}\right)+\bar{Q}\left(p_{2}\right)+q\left(p_{3}\right)+\bar{q}\left(p_{4}\right) ;$ and $(\mathrm{c}) e^{+} e^{-} \rightarrow Q\left(p_{1}\right)+\bar{Q}\left(p_{2}\right)+$ $Q\left(p_{3}\right)+\bar{Q}\left(p_{4}\right)$. The quark $q$ could be any flavour other than $Q$, massless or massive. Since the matrix elements, $\mathcal{M}_{4}$, for these processes are rather lengthy, it is not feasible to explicitly show the cancellation of the soft and collinear poles between the real-emission matrix elements and the auxiliary cross sections constructed from various dipole contributions. Therefore we only give the parts of the auxiliary cross sections, but do not reproduce $\mathcal{M}_{4}$, which can be be found in Ref. [57].

For process (a), we have to evaluate ten dipole contributions, $\mathcal{D}_{31,2}, \mathcal{D}_{31,4}, \mathcal{D}_{41,2}, \mathcal{D}_{41,3}$, $\mathcal{D}_{32,1}, \mathcal{D}_{32,4}, \mathcal{D}_{42,1}, \mathcal{D}_{42,3}, \mathcal{D}_{34,1}$ and $\mathcal{D}_{34,2}$. The associated colour algebra is again straightforward because the different colour projections of the three-parton matrix element fully factorize (see Appendix A of Ref. [11]). Thus we do not need to calculate any colourcorrelated tree amplitudes and we obtain

$$
\begin{aligned}
& \mathcal{D}_{31,2}^{(\mathrm{a})}\left(p_{1}, p_{2}, p_{3}, p_{4}\right)=\frac{1}{2 p_{3} \cdot p_{1}}\left(1-\frac{C_{\mathrm{A}}}{2 C_{\mathrm{F}}}\right)\left\langle\mathbf{V}_{g_{3} Q_{1}, 2}\right\rangle\left|\mathcal{M}_{3}\left(\widetilde{p}_{31}, \widetilde{p}_{2}, p_{4}\right)\right|^{2}, \\
& \mathcal{D}_{31,4}^{(\mathrm{a})}\left(p_{1}, p_{2}, p_{3}, p_{4}\right)=\frac{1}{2 p_{3} \cdot p_{1}} \frac{C_{\mathrm{A}}}{2 C_{\mathrm{F}}}\left\langle\mathbf{V}_{g_{3} Q_{1}, 4}\right\rangle\left|\mathcal{M}_{3}\left(\widetilde{p}_{31}, p_{2}, \widetilde{p}_{4}\right)\right|^{2}, \\
& \mathcal{D}_{34,1}^{(\mathrm{a})}\left(p_{1}, p_{2}, p_{3}, p_{4}\right)=\frac{1}{2 p_{3} \cdot p_{4}} \frac{1}{2}\left\langle\mu\left|\mathbf{V}_{g_{3} g_{4}, 1}\right| \nu\right\rangle \mathcal{T}_{\mu \nu}\left(\widetilde{p}_{1}, p_{2}, \widetilde{p}_{34}\right) .
\end{aligned}
$$

The dipole contributions $\mathcal{D}_{32,1}, \mathcal{D}_{32,4}$ and $\mathcal{D}_{34,2}$ can be obtained from $\mathcal{D}_{31,2}, \mathcal{D}_{31,4}$ and $\mathcal{D}_{34,1}$ respectively by the replacement $p_{1} \leftrightarrow p_{2}$. Likewise $\mathcal{D}_{41,2}$ and $\mathcal{D}_{41,3}$ can be obtained from $\mathcal{D}_{31,2}$ and $\mathcal{D}_{31,4}$ respectively by the replacement $p_{3} \leftrightarrow p_{4}$. Finally, $\mathcal{D}_{42,1}$ and $\mathcal{D}_{42,3}$ can be obtained from $\mathcal{D}_{31,2}$ and $\mathcal{D}_{31,4}$ respectively by the replacement $p_{1} \leftrightarrow p_{2}$ and $p_{3} \leftrightarrow p_{4}$.

The splitting functions $\left\langle\mathbf{V}_{g_{3} Q_{1}, 2}\right\rangle$ and $\left\langle\mathbf{V}_{g_{3} Q_{1}, 4}\right\rangle$ are given by Eq. (5.16) with $m_{k}=m_{Q}$ and $m_{k}=0$ respectively and $\left\langle\mu\left|\mathbf{V}_{g_{3} g_{4}, 1}\right| \nu\right\rangle$ by Eq. (5.19) with $m_{k}=m_{Q}$. The tensor $\mathcal{T}_{\mu \nu}$ is given below. 
For process (b) we have to calculate at most four dipole contributions, $\mathcal{D}_{34,1}$ and $\mathcal{D}_{34,2}$ and, perhaps, $\mathcal{D}_{12,3}$ and $\mathcal{D}_{12,4}$. We obtain

$$
\mathcal{D}_{34,1}^{(\mathrm{b})}\left(p_{1}, p_{2}, p_{3}, p_{4}\right)=\frac{1}{2 p_{3} \cdot p_{4}+2 m_{q}^{2}} \frac{1}{2}\left\langle\mu\left|\mathbf{V}_{q_{3} \bar{q}_{4}, 1}\right| \nu\right\rangle \mathcal{T}_{\mu \nu}\left(\widetilde{p}_{1}, p_{2}, \widetilde{p}_{34}\right)
$$

The dipole contribution $\mathcal{D}_{34,2}$ can be obtained from $\mathcal{D}_{34,1}$ by the replacement $p_{1} \leftrightarrow p_{2}$. The splitting function $\left\langle\mu\left|\mathbf{V}_{q_{3} \bar{q}_{4}, 1}\right| \nu\right\rangle$ is given by Eq. (5.17) with $m_{k}=m_{Q}$. Note that we have explicitly kept $m_{q}$ non-zero in Eq. (D.33). Since all our results tend smoothly to the massless results in the small-mass limit, we can easily replace $m_{q}$ by zero if necessary.

The kinematics for the dipole contributions $\mathcal{D}_{12,3}$ and $\mathcal{D}_{12,4}$ replace the heavy quark and antiquark momenta $p_{1}$ and $p_{2}$ by a massless gluon with momentum $\widetilde{p}_{12}$. If our observable, $F_{J}$, requires the presence of heavy quarks in the final state then it will get zero contribution from these dipole contributions, leaving uncancelled logarithms of $\mu_{Q}^{2}$ in the 4-parton integral. Even in this case, it may be helpful to replace the observable by a pseudo-observable in which the subtraction $q \bar{q} g$ configurations do contribute, improving the numerical convergence of the three-parton integral and allowing the logarithms to be isolated in the pseudo-collinear region. This may be more amenable to analytical treatment, allowing the logarithms to be summed to all orders [58]. If the observable sums over all final states, then $\mathcal{D}_{12,3}$ and $\mathcal{D}_{12,4}$ are needed. If necessary, they can be obtained from $\mathcal{D}_{34,1}$ and $\mathcal{D}_{34,2}$ by the replacements $p_{1} \leftrightarrow p_{3}, p_{2} \leftrightarrow p_{4}$ and $m_{Q} \leftrightarrow m_{q}$.

For process (c) we have to calculate eight dipole contributions, $\mathcal{D}_{12,3}, \mathcal{D}_{12,4}, \mathcal{D}_{14,2}, \mathcal{D}_{14,3}$, $\mathcal{D}_{23,1}, \mathcal{D}_{23,4}, \mathcal{D}_{34,1}$ and $\mathcal{D}_{34,2}$. They are identical to those for process (b), with $m_{q}$ replaced by $m_{Q}$. Note however that the external factors in Eq. (6.4) introduce an extra factor of $\frac{1}{4}$ coming from the counting of two pairs of identical particles in the final state. The other dipoles can be obtained by appropriate permutations of momenta.

The tensor $\mathcal{T}_{\mu \nu}$ appearing in Eqs. (D.32,D.33) is the squared amplitude for the LO process $e^{+} e^{-} \rightarrow Q \bar{Q} g$ not summed over the gluon polarization. It is normalized so that $-g^{\mu \nu} \mathcal{T}_{\mu \nu}=\left|\mathcal{M}_{3}\right|^{2}$. Again averaging over event orientation and polarization and neglecting terms that are antisymmetric in $\mu, \nu$, which cannot contribute to the cross section, we obtain

$$
\mathcal{T}_{\mu \nu}\left(p_{1}, p_{2}, p_{3}\right)=-C_{\mathrm{F}} \frac{8 \pi \alpha_{\mathrm{s}}}{s} \frac{\left(g^{V V}+g^{A A}\right) \mathcal{T}_{\mu \nu}^{V V}+g^{A A} \mu_{Q}^{2} \mathcal{T}_{\mu \nu}^{A A-V V}}{\left(1-x_{1}\right)\left(1-x_{2}\right)}
$$

with

$$
\begin{aligned}
\mathcal{T}_{\mu \nu}^{V V}= & 2\left(1+2 \mu_{Q}^{2}\right)\left[\frac{p_{1}^{\mu} p_{2}^{\nu}}{s}+\frac{p_{2}^{\mu} p_{1}^{\nu}}{s}\right]-2 \frac{1-x_{1}}{1-x_{2}}\left(1+2 \mu_{Q}^{2}\right) \frac{p_{1}^{\mu} p_{1}^{\nu}}{s}-2 \frac{1-x_{2}}{1-x_{1}}\left(1+2 \mu_{Q}^{2}\right) \frac{p_{2}^{\mu} p_{2}^{\nu}}{s} \\
& -\frac{1-x_{1}-x_{2}+x_{2}^{2}-2 \mu_{Q}^{2}\left(x_{1}-x_{2}\right)}{1-x_{2}}\left[\frac{p_{1}^{\mu} p_{3}^{\nu}}{s}+\frac{p_{3}^{\mu} p_{1}^{\nu}}{s}\right] \\
& -\frac{1-x_{2}-x_{1}+x_{1}^{2}-2 \mu_{Q}^{2}\left(x_{2}-x_{1}\right)}{1-x_{1}}\left[\frac{p_{2}^{\mu} p_{3}^{\nu}}{s}+\frac{p_{3}^{\mu} p_{2}^{\nu}}{s}\right] \\
& +\left(1+\frac{1}{2} x_{1}^{2}+\frac{1}{2} x_{2}^{2}-x_{1}-x_{2}\right) g^{\mu \nu}+4 \mu_{Q}^{2} \frac{p_{3}^{\mu} p_{3}^{\nu}}{s}, \\
\mathcal{T}_{\mu \nu}^{A A-V V}= & -12\left[\frac{p_{1}^{\mu} p_{2}^{\nu}}{s}+\frac{p_{2}^{\mu} p_{1}^{\nu}}{s}\right]+12 \frac{1-x_{1}}{1-x_{2}} \frac{p_{1}^{\mu} p_{1}^{\nu}}{s}+12 \frac{1-x_{2}}{1-x_{1}} \frac{p_{2}^{\mu} p_{2}^{\nu}}{s}
\end{aligned}
$$




$$
\begin{aligned}
& -2 \frac{2+2 x_{1}-6 x_{2}+x_{1} x_{2}+x_{2}^{2}}{1-x_{2}}\left[\frac{p_{1}^{\mu} p_{3}^{\nu}}{s}+\frac{p_{3}^{\mu} p_{1}^{\nu}}{s}\right] \\
& -2 \frac{2+2 x_{2}-6 x_{1}+x_{1} x_{2}+x_{1}^{2}}{1-x_{1}}\left[\frac{p_{2}^{\mu} p_{3}^{\nu}}{s}+\frac{p_{3}^{\mu} p_{2}^{\nu}}{s}\right] \\
& +\left(2-x_{1}-x_{2}\right)^{2} g^{\mu \nu}-4\left(3-x_{1}-x_{2}\right) \frac{p_{3}^{\mu} p_{3}^{\nu}}{s} .
\end{aligned}
$$

Next we have to evaluate the insertion operator $\boldsymbol{I}(\epsilon)$, which gives the integral of the auxiliary cross section, and combine it with the virtual cross section, which was calculated in Refs. [34, 59]. We use the notation of Ref. [59]. They obtain

$$
\begin{aligned}
\left|\mathcal{M}_{3}\left(p_{1}, p_{2}, p_{3}\right)\right|_{(1-\text { loop })=}^{2}= & -\frac{\alpha_{\mathrm{s}}}{4 \pi} N_{\mathrm{c}}\left\{\frac{2}{\epsilon^{2}}+\frac{1}{\epsilon}\left[\frac{17}{3}+2\left(\ln \frac{4 \pi \mu^{2}}{s}+\ln \frac{\mu_{Q}^{2}}{\left(1-x_{1}\right)\left(1-x_{2}\right)}-\gamma_{E}\right)\right.\right. \\
& \left.\left.-\frac{2 N_{f}}{3 N_{\mathrm{c}}}-\frac{1}{N_{\mathrm{c}}^{2}} \frac{1}{\beta}\left(2 \beta-\left(1+\beta^{2}\right) \ln \frac{1+\beta}{1-\beta}\right)\right]\right\}\left|\mathcal{M}_{3}^{(4)}\left(p_{1}, p_{2}, p_{3}\right)\right|^{2} \\
& +\frac{\alpha_{\mathrm{s}}}{2 \pi} N_{\mathrm{c}} \frac{1}{\epsilon}\left|\mathcal{M}_{3}^{(\prime)}\left(p_{1}, p_{2}, p_{3}\right)\right|^{2}+F_{\text {finite }}\left(p_{1}, p_{2}, p_{3}\right),
\end{aligned}
$$

where $N_{f}$ is the number of massless flavours and $\beta=\sqrt{1-4 \mu_{Q}^{2} /\left(x_{1}+x_{2}-1\right)}$ is the velocity of $p_{1}$ in the $p_{1}+p_{2}$ frame. The function $F_{\text {finite }}$ contains all of the finite (as $\epsilon \rightarrow 0$ ) terms,

$$
F_{\text {finite }}=C_{\mathrm{F}} \frac{8 \pi \alpha_{\mathrm{s}}}{s} \times 2\left(g^{V V} F_{\text {finite }}^{V V}+g^{A A} F_{\text {finite }}^{A A}\right),
$$

where

$$
F_{\text {finite }}^{V V / A A}=F_{1}^{\mathrm{counter}, \text { finite }, V V / A A}+F_{1}^{\mathrm{ext} ., \mathrm{finite}, V V / A A}+\frac{\alpha_{\mathrm{s}}}{4 \pi} N_{\mathrm{c}}\left\{F_{1}^{\mathrm{lc}, V V / A A}+\frac{1}{N_{\mathrm{c}}^{2}} F_{1}^{\mathrm{sc}, V V / A A}\right\},
$$

with $F_{1}^{\text {counter,finite, } V V / A A}, F_{1}^{\text {ext.,finite, } V V / A A}, F_{1}^{\text {lc, } V V / A A}$ and $F_{1}^{\mathrm{sc}, V V / A A}$ defined in Eqs. (4.11), (A.2) and (A.3) of Ref. [59]. In Eqs. (D.37D.39) the expansion parameter is $\alpha_{\mathrm{s}}^{\overline{\mathrm{MS}}}$, i.e. charge renormalization is carried out in the $\overline{\mathrm{MS}}$ subtraction scheme.

In checking the quasi-collinear limit of our formulae, it is useful to note the logarithmic terms in $F_{\text {finite }}$. We obtain间

$$
\begin{aligned}
F_{\text {finite }}\left(p_{1}, p_{2}, p_{3}\right)=\frac{\alpha_{\mathrm{s}}}{2 \pi}\left|\mathcal{M}_{3}^{(4)}\right|^{2}\left\{\ln \frac{\mu^{2}}{s}\left(\frac{11}{6} C_{\mathrm{A}}-\frac{2}{3} T_{\mathrm{R}}\left(N_{f}+N_{F}\right)\right)\right. \\
\quad-\frac{1}{2} C_{\mathrm{A}}\left(\gamma_{E}-\ln \frac{4 \pi \mu^{2}}{s}\right)^{2}+\frac{2 C_{\mathrm{F}}-C_{\mathrm{A}}}{v_{Q \bar{Q}}} \ln \sqrt{\frac{1-v_{Q \bar{Q}}}{1+v_{Q \bar{Q}}}}\left(\gamma_{E}-\ln \frac{4 \pi \mu^{2}}{s}\right) \\
+\left(\gamma_{E}-\ln \frac{4 \pi \mu^{2}}{s}\right)\left(2 C_{\mathrm{F}}+\frac{11}{6} C_{\mathrm{A}}-\frac{2}{3} T_{\mathrm{R}} N_{f}+C_{\mathrm{A}} \ln \frac{s m_{Q}^{2}}{s_{Q g} s_{\bar{Q} g}}\right) \\
\left.+C_{\mathrm{F}} \ln ^{2} \mu_{Q}^{2}-C_{\mathrm{F}} \ln \mu_{Q}^{2}+\frac{2}{3} T_{\mathrm{R}} \sum_{F=1}^{N_{F}} \ln \frac{m_{F}^{2}}{s}\right\} \\
+\frac{\alpha_{\mathrm{s}}}{2 \pi}\left|\mathcal{M}_{3}^{(\prime)}\right|^{2}\left\{-C_{\mathrm{A}}\left(\gamma_{E}-\ln \frac{4 \pi \mu^{2}}{s}\right)+2 C_{\mathrm{F}} \ln \mu_{Q}^{2}\right\}+\ldots
\end{aligned}
$$

\footnotetext{
${ }^{\dagger \dagger}$ Note that the result in Ref. [59] is for a single heavy flavour. We have trivially modified it to incorporate $N_{F}$ heavy flavours, which simply involves changing the logarithmic term in $F_{1}^{\text {ext.,finite, } V V / A A}$ from $\ln \left(z s / \mu^{2}\right)$ to $\sum_{F=1}^{N_{F}} \ln \left(m_{F}^{2} / \mu^{2}\right)$.
} 
where the ellipsis represents terms that stay finite or vanish for $\mu_{Q}^{2} \rightarrow 0$ and $m_{F}^{2} / s \rightarrow 0$. The first line comes from the renormalization of $\alpha_{s}$, with $\mu$ the renormalization scale, and will remain in the final result. All the other occurrences of $\mu$ come from the trivial fact that in Ref. [59], the natural overall factor of $\left(4 \pi \mu^{2} / s\right)^{\epsilon} / \Gamma(1-\epsilon)$ is expanded in $\epsilon$ and are cancelled by our insertion operator, $\boldsymbol{I}(\epsilon)$. All the remaining mass logarithms are related to the quasi-collinear limit and should also be cancelled by $\boldsymbol{I}(\epsilon)$.

The general expression for $\boldsymbol{I}(\epsilon)$ is given in Eq. (6.16). The colour structure again factorizes and we obtain

$$
\begin{aligned}
{ }_{3}\left\langle 1,2,3\left|\boldsymbol{I}_{3}\left(\epsilon, \mu^{2} ;\left\{p_{i}, m_{i}\right\}\right)\right| 1,2,3\right\rangle_{3}=\left|\mathcal{M}_{3}^{(d)}\right|^{2} \times \frac{\alpha_{\mathrm{S}}}{2 \pi} \frac{(4 \pi)^{\epsilon}}{\Gamma(1-\epsilon)}\left\{C_{\mathrm{A}}\left(\frac{\mu^{2}}{s_{Q g}}\right)^{\epsilon} \mathcal{V}^{(\mathrm{S})}\left(s_{Q g}, m_{Q}, 0 ; \epsilon\right)\right. \\
+C_{\mathrm{A}}\left(\frac{\mu^{2}}{s_{\bar{Q} g}}\right)^{\epsilon} \mathcal{V}^{(\mathrm{S})}\left(s_{\bar{Q} g}, m_{Q}, 0 ; \epsilon\right)+\left(2 C_{\mathrm{F}}-C_{\mathrm{A}}\right)\left(\frac{\mu^{2}}{s_{Q \bar{Q}}}\right)^{\epsilon} \mathcal{V}^{(\mathrm{S})}\left(s_{Q \bar{Q}}, m_{Q}, m_{Q} ; \epsilon\right) \\
+\frac{C_{\mathrm{A}}}{2} \mathcal{V}_{Q}^{(\mathrm{NS})}\left(s_{Q g}, m_{Q}, 0\right)+\frac{2 C_{\mathrm{F}}-C_{\mathrm{A}}}{2} \mathcal{V}_{Q}^{(\mathrm{NS})}\left(s_{Q \bar{Q}}, m_{Q}, m_{Q}\right) \\
+\frac{C_{\mathrm{A}}}{2} \mathcal{V}_{Q}^{(\mathrm{NS})}\left(s_{\bar{Q} g}, m_{Q}, 0\right)+\frac{2 C_{\mathrm{F}}-C_{\mathrm{A}}}{2} \mathcal{V}_{Q}^{(\mathrm{NS})}\left(s_{Q \bar{Q}}, m_{Q}, m_{Q}\right) \\
+\frac{C_{\mathrm{A}}}{2} \mathcal{V}_{g}^{(\mathrm{NS})}\left(s_{Q g}, 0, m_{Q},\left\{m_{F}\right\} ; \kappa\right)+\frac{C_{\mathrm{A}}}{2} \mathcal{V}_{g}^{(\mathrm{NS})}\left(s_{\bar{Q} g}, 0, m_{Q},\left\{m_{F}\right\} ; \kappa\right) \\
+\frac{C_{\mathrm{A}}}{2 C_{\mathrm{F}}} \gamma_{q} \ln \frac{\mu^{2}}{s_{Q g}}+\frac{1}{2} \gamma_{g} \ln \frac{\mu^{2}}{s_{Q g}}+\left(1-\frac{C_{\mathrm{A}}}{2 C_{\mathrm{F}}}\right) \gamma_{q} \ln \frac{\mu^{2}}{s_{Q \bar{Q}}} \\
+\frac{C_{\mathrm{A}}}{2 C_{\mathrm{F}}} \gamma_{q} \ln \frac{\mu^{2}}{s_{\bar{Q} g}}+\frac{1}{2} \gamma_{g} \ln \frac{\mu^{2}}{s_{\bar{Q} g}}+\left(1-\frac{C_{\mathrm{A}}}{2 C_{\mathrm{F}}}\right) \gamma_{q} \ln \frac{\mu^{2}}{s_{Q \bar{Q}}} \\
+\left(2 C_{\mathrm{F}}+C_{\mathrm{A}} \frac{\pi^{2}}{3}+2 \Gamma_{Q}\left(\mu, m_{Q} ; \epsilon\right)+2 \gamma_{q}+2 K_{q}+\Gamma_{g}\left(\left\{m_{F}\right\} ; \epsilon\right)+\gamma_{g}+K_{g}\right\}
\end{aligned}
$$

where $s_{j k}=2 p_{j} \cdot p_{k}, \mathcal{V}^{(\mathrm{S})}$ is defined in Eq. (6.20), $\mathcal{V}_{j}^{(\mathrm{NS})}$ are defined in Eqs. (6.21 6.26), $\Gamma_{j}$ are defined in Eqs. (6.27 6.29) and $\gamma_{j}$ and $K_{j}$ are defined in Eqs. (5.91) and (6.17).

It is worth stressing that Eq. (D.41) is the insertion operator for the $Q \bar{Q} g$ final state. It does not therefore account for terms coming from the splitting process $g \rightarrow Q \bar{Q}$ in process (b) above, which contribute to the $q \bar{q} g$ final state. The corresponding insertion operator can be obtained by replacing $m_{Q} \rightarrow m_{q}$ in Eq. (D.41), while retaining $m_{Q}$ in the set $\left\{m_{F}\right\}$.

Explicitly, we obtain

$$
\begin{aligned}
& { }_{3}\left\langle 1,2,3\left|\boldsymbol{I}_{3}\left(\epsilon, \mu^{2} ;\left\{p_{i}, m_{i}\right\}\right)\right| 1,2,3\right\rangle_{3}=\left|\mathcal{M}_{3}^{(d)}\right|^{2} \times \frac{\alpha_{\mathrm{s}}}{2 \pi} \frac{1}{\Gamma(1-\epsilon)}\left(\frac{4 \pi \mu^{2}}{s}\right)^{\epsilon}\{ \\
& C_{\mathrm{A}}\left(\frac{y_{Q g}^{-\epsilon}}{2 \epsilon^{2}}+\frac{y_{Q g}^{-\epsilon}}{2 \epsilon} \ln \frac{m_{Q}^{2}}{s_{Q g}}\right)+C_{\mathrm{A}}\left(\frac{y_{\bar{Q} g}^{-\epsilon}}{2 \epsilon^{2}}+\frac{y_{\bar{Q} g}^{-\epsilon}}{2 \epsilon} \ln \frac{m_{Q}^{2}}{s_{\bar{Q} g}}\right)+\frac{2}{\epsilon} C_{\mathrm{F}}+\frac{1}{\epsilon}\left(\frac{11}{6} C_{\mathrm{A}}-\frac{2}{3} T_{\mathrm{R}} N_{f}\right) \\
& +\frac{2 C_{\mathrm{F}}-C_{\mathrm{A}}}{v_{Q \bar{Q}}} \frac{y_{Q \bar{Q}}^{-\epsilon}}{\epsilon} \ln \sqrt{\frac{1-v_{Q \bar{Q}}}{1+v_{Q \bar{Q}}}}-\frac{1}{4} C_{\mathrm{A}} \ln ^{2} \frac{m_{Q}^{2}}{s_{Q g}}-\frac{1}{4} C_{\mathrm{A}} \ln ^{2} \frac{m_{Q}^{2}}{s_{\bar{Q} g}} \\
& -\frac{1}{2} \frac{2 C_{\mathrm{F}}-C_{\mathrm{A}}}{v_{Q \bar{Q}}} \ln ^{2} \sqrt{\frac{1-v_{Q \bar{Q}}}{1+v_{Q \bar{Q}}}}-\frac{2}{3} T_{\mathrm{R}} \sum_{F=1}^{N_{F}} \ln \frac{m_{F}^{2}}{Q_{\mathrm{aux}}^{2}}+C_{\mathrm{F}} \ln \frac{m_{Q}^{2}}{s}
\end{aligned}
$$




$$
\left.+G\left(p_{1}, p_{2}, p_{3}\right)\right\},
$$

where $y_{j k}=s_{j k} / s$ and $Q_{\text {aux }}$ is an arbitrary scale that cancels against an equivalent term in $G$, as discussed after Eq. (6.24). The function $G$ contains the terms that do not diverge as either $\epsilon \rightarrow 0$ or $m_{F}^{2} \ll s$,

$$
\begin{aligned}
& G\left(p_{1}, p_{2}, p_{3}\right)=\frac{3}{4} C_{\mathrm{A}} \ln \frac{s_{Q \bar{Q}}^{2}}{s_{Q g} s_{\bar{Q} g}}+3 C_{\mathrm{F}} \ln \frac{s}{s_{Q \bar{Q}}}+\frac{1}{2}\left(\frac{11}{6} C_{\mathrm{A}}-\frac{2}{3} T_{\mathrm{R}} N_{f}\right) \ln \frac{s^{2}}{s_{Q g} s_{\bar{Q} g}} \\
& +6 C_{\mathrm{F}}+\frac{50}{9} C_{\mathrm{A}}-\frac{16}{9} T_{\mathrm{R}} N_{f}-C_{\mathrm{F}} \pi^{2}-\frac{2}{3} C_{\mathrm{A}} \pi^{2}-\frac{2 C_{\mathrm{F}}-C_{\mathrm{A}}}{v_{Q \bar{Q}}} \frac{\pi^{2}}{6} \\
& \quad-C_{\mathrm{A}} \frac{1}{2} \ln \frac{m_{Q}^{4}}{s_{Q g}\left(s_{Q g}+m_{Q}^{2}\right)} \ln \frac{s_{Q g}}{s_{Q g}+m_{Q}^{2}}-C_{\mathrm{A}} \frac{1}{2} \ln \frac{m_{Q}^{4}}{s_{\bar{Q} g}\left(s_{\bar{Q} g}+m_{Q}^{2}\right)} \ln \frac{s_{\bar{Q} g}}{s_{\bar{Q} g}+m_{Q}^{2}} \\
& +\frac{2 C_{\mathrm{F}}-C_{\mathrm{A}}}{v_{Q \bar{Q}}} \ln \sqrt{\frac{1-v_{Q \bar{Q}}}{1+v_{Q \bar{Q}}}} \ln \frac{s_{Q \bar{Q}}+2 m_{Q}^{2}}{s_{Q \bar{Q}}} \\
& +\frac{C_{\mathrm{A}}}{2} \mathcal{V}_{Q}^{(\mathrm{NS})}\left(s_{Q g}, m_{Q}, 0\right)+\frac{C_{\mathrm{A}}}{2} \mathcal{V}_{Q}^{(\mathrm{NS})}\left(s_{\bar{Q} g}, m_{Q}, 0\right)+\left(2 C_{\mathrm{F}}-C_{\mathrm{A}}\right) \mathcal{V}_{Q}^{(\mathrm{NS})}\left(s_{Q \bar{Q}}, m_{Q}, m_{Q}\right) \\
& +\frac{C_{\mathrm{A}}}{2} \mathcal{V}_{g}^{(\mathrm{NS})}\left(s_{Q g}, 0, m_{Q},\left\{m_{F}\right\} ; \kappa\right)+\frac{C_{\mathrm{A}}}{2} \mathcal{V}_{g}^{(\mathrm{NS})}\left(s_{\bar{Q} g}, 0, m_{Q},\left\{m_{F}\right\} ; \kappa\right) .
\end{aligned}
$$

Combining Eqs. (D.37) and (D.42), we obtain

$$
\begin{aligned}
{ }_{3}\left\langle 1,2,3\left|\boldsymbol{I}_{3}\left(\epsilon, \mu^{2} ;\left\{p_{i}, m_{i}\right\}\right)\right| 1,2,3\right\rangle_{3}+\left|\mathcal{M}_{3}\left(p_{1}, p_{2}, p_{3}\right)\right|_{(1-\text { loop })]_{\epsilon=0}}^{2} & = \\
\frac{\alpha_{\mathrm{s}}}{2 \pi}\left|\mathcal{M}_{3}^{(4)}\right|^{2} & \left\{\frac{1}{2} C_{\mathrm{A}}\left(\gamma_{E}-\ln \frac{4 \pi \mu^{2}}{s}\right)^{2}-\frac{2 C_{\mathrm{F}}-C_{\mathrm{A}}}{v_{Q \bar{Q}}} \ln \sqrt{\frac{1-v_{Q \bar{Q}}}{1+v_{Q \bar{Q}}}}\left(\gamma_{E}-\ln \frac{4 \pi \mu^{2}}{s_{Q \bar{Q}}}\right)\right. \\
& -\left(\gamma_{E}-\ln \frac{4 \pi \mu^{2}}{s}\right)\left(2 C_{\mathrm{F}}+\frac{11}{6} C_{\mathrm{A}}-\frac{2}{3} T_{\mathrm{R}} N_{f}+C_{\mathrm{A}} \ln \frac{s m_{Q}^{2}}{s_{Q g} s_{\bar{Q} g}}\right) \\
+ & C_{\mathrm{A}}\left(\frac{1}{4} \ln ^{2} y_{Q g}+\frac{1}{4} \ln ^{2} y_{\bar{Q} g}-\frac{1}{2} \ln y_{Q g} \ln \frac{m_{Q}^{2}}{s_{Q g}}-\frac{1}{2} \ln y_{\bar{Q} g} \ln \frac{m_{Q}^{2}}{s_{\bar{Q} g}}-\frac{\pi^{2}}{12}\right) \\
& -\frac{1}{4} C_{\mathrm{A}} \ln ^{2} \frac{m_{Q}^{2}}{s_{Q g}}-\frac{1}{4} C_{\mathrm{A}} \ln ^{2} \frac{m_{Q}^{2}}{s_{\bar{Q} g}}-\frac{1}{2} \frac{2 C_{\mathrm{F}}-C_{\mathrm{A}}}{v_{Q \bar{Q}}} \ln { }^{2} \sqrt{\frac{1-v_{Q \bar{Q}}}{1+v_{Q \bar{Q}}}} \\
& \left.-\frac{2}{3} T_{\mathrm{R}} \sum_{F=1}^{N_{F}} \ln \frac{m_{F}^{2}}{Q_{\mathrm{aux}}^{2}}+C_{\mathrm{F}} \ln \frac{m_{Q}^{2}}{s}+G\left(p_{1}, p_{2}, p_{3}\right)\right\} \\
+\frac{\alpha_{\mathrm{s}}}{2 \pi}\left|\mathcal{M}_{3}^{(\prime)}\right|^{2}\{ & -2 C_{\mathrm{F}}-\left(\frac{11}{6} C_{\mathrm{A}}-\frac{2}{3} T_{\mathrm{R}} N_{f}\right)-\frac{2 C_{\mathrm{F}}-C_{\mathrm{A}}}{v_{Q \bar{Q}}} \ln \sqrt{\frac{1-v_{Q \bar{Q}}}{1+v_{Q \bar{Q}}}} \\
& \left.-C_{\mathrm{A}}\left(\ln \frac{4 \pi \mu^{2} m_{Q}^{2}}{s_{Q g} s_{\bar{Q} g}}-\gamma_{E}\right)\right\} \\
+F_{\text {finite }}\left(p_{1},\right. & \left.p_{2}, p_{3}\right) .
\end{aligned}
$$

The integration of this expression over the phase space in Eq. (D.8) with the observable $F_{J}^{(3)}$ gives the three-parton cross section $\sigma^{\mathrm{NLO}\{3\}}$.

Comparing Eqs. (D.44) and (D.40), we see that all logarithms of $m_{Q}^{2}$ cancel, leaving a cross section that is well-behaved for all masses. 
If the observable to be calculated receives contributions from all final states, whether containing a heavy quark or not, it is also necessary to calculate the three-parton cross section for light quarks. Since our formalism guarantees a smooth small-mass limit, it is straightforward to set $m_{Q}$ to zero in Eq. (D.44), while keeping $m_{F}$ non-zero.

\section{D.3 ep $\rightarrow e Q \bar{Q} X$}

Since the analytical formulae are more lengthy in this caseff, we only give the formulae necessary to construct the auxiliary cross section and its integral.

For simplicity, we start by assuming that the observable of interest requires the presence of a heavy quark pair in the final state. That is, we do not consider heavy-quark corrections to light-quark processes. Thus, at lowest order, there is only one process, $e+g\left(p_{a}\right) \rightarrow$ $e+Q\left(p_{1}\right)+\bar{Q}\left(p_{2}\right)$, with colour averaged matrix element $1 /\left(N_{\mathrm{c}}^{2}-1\right)\left|\mathcal{M}_{3}\left(p_{1}, p_{2},-p_{a}\right)\right|^{2}$. Note that this matrix element is not identical to the one given earlier for the process $e^{+} e^{-} \rightarrow Q \bar{Q} g$, only because we averaged over the orientation of the three-jet system in the $e^{+} e^{-}$rest frame. If the full angular information had been retained, then $\left|\mathcal{M}_{3}\right|^{2}$ could simply be obtained by crossing the $e^{+} e^{-}$annihilation result. The same comment applies to the tensor $\mathcal{T}_{\mu \nu}$, to be defined shortly.

Three NLO real emission processes contribute: (a) $e+g\left(p_{a}\right) \rightarrow e+Q\left(p_{1}\right)+\bar{Q}\left(p_{2}\right)+g\left(p_{3}\right)$; (b) $e+q\left(p_{a}\right) \rightarrow e+Q\left(p_{1}\right)+\bar{Q}\left(p_{2}\right)+q\left(p_{3}\right)$; and $(\mathrm{c}) e+\bar{q}\left(p_{a}\right) \rightarrow e+Q\left(p_{1}\right)+\bar{Q}\left(p_{2}\right)+\bar{q}\left(p_{3}\right)$. The last is identical to the second so we do not explicitly consider it further.

For process (a), we have to evaluate six dipole contributions, $\mathcal{D}_{31,2}, \mathcal{D}_{32,1}, \mathcal{D}_{31}^{a}, \mathcal{D}_{32}^{a}, \mathcal{D}_{1}^{a 3}$ and $\mathcal{D}_{2}^{a 3}$. The first two are constructed in exactly the same way as in the $e^{+} e^{-}$processes already considered. The third dipole contribution, $\mathcal{D}_{31}^{a}$, with a final-state emitter and initial-state spectator, is given by

$$
\mathcal{D}_{31}^{a(\mathrm{a})}=\frac{1}{2 p_{3} \cdot p_{1}} \frac{C_{\mathrm{A}}}{2 C_{\mathrm{F}}} \frac{1}{x_{31, a}}\left\langle\mathbf{V}_{g_{3} Q_{1}}^{a}\right\rangle \times \frac{1}{N_{\mathrm{c}}^{2}-1}\left|\mathcal{M}_{3}\left(\widetilde{p}_{31}, p_{2},-\widetilde{p}_{a}\right)\right|^{2},
$$

with

$$
\begin{gathered}
x_{31, a}=\frac{p_{a} \cdot p_{3}+p_{a} \cdot p_{1}-p_{3} \cdot p_{1}}{p_{a} \cdot p_{3}+p_{a} \cdot p_{1}}, \\
\widetilde{p}_{a}^{\mu}=x_{31, a} p_{a}^{\mu}, \quad \widetilde{p}_{31}^{\mu}=p_{3}^{\mu}+p_{1}^{\mu}-\left(1-x_{31, a}\right) p_{a}^{\mu},
\end{gathered}
$$

and $\left\langle\mathbf{V}_{g_{3} Q_{1}}^{a}\right\rangle$ in Eq. (5.50). The dipole contribution $\mathcal{D}_{32}^{a}$ can be obtained from $\mathcal{D}_{31}^{a}$ by the replacement $p_{1} \leftrightarrow p_{2}$.

The fifth dipole contribution for process (a), $\mathcal{D}_{1}^{a 3}$, with an initial-state emitter and final-state spectator, is given by

$$
\mathcal{D}_{1}^{a 3(\mathrm{a})}=\frac{1}{2 p_{a} \cdot p_{3}} \frac{1}{2} \frac{1}{x_{31, a}}\left\langle\mu\left|\mathbf{V}_{1}^{g_{a} g_{3}}\right| \nu\right\rangle \times \frac{1}{N_{\mathrm{c}}^{2}-1} \mathcal{T}_{\mu \nu}\left(\widetilde{p}_{31}, p_{2},-\widetilde{p}_{a}\right),
$$

$\ddagger$ A NLO Monte Carlo program, based on the subtraction method, to compute infrared and collinear safe observables in this process is presented in Ref. [60]. 
with $x_{31, a}, \widetilde{p}_{a}$ and $\widetilde{p}_{31}$ given by Eqs. (D.46) and (D.47) and $\left\langle\mu\left|\mathbf{V}_{1}^{g_{a} g_{3}}\right| \nu\right\rangle$ given in Eq. (5.85). The tensor $\mathcal{T}_{\mu \nu}$ is the squared amplitude for the LO process not summed over gluon polarization, normalized so that $-g^{\mu \nu} \mathcal{T}_{\mu \nu}=\left|\mathcal{M}_{3}\right|^{2}$. The dipole contribution $\mathcal{D}_{2}^{a 3}$ can be obtained from $\mathcal{D}_{1}^{a 3}$ by the replacement $p_{1} \leftrightarrow p_{2}$.

In process (b), we only have to evaluate two dipole contributions, $\mathcal{D}_{1}^{a 3}$ and $\mathcal{D}_{2}^{a 3}$ (recall that we assume for now that our observable requires heavy quarks in the final state. The other two dipoles for this process, $\mathcal{D}_{12,3}$ and $\mathcal{D}_{12}^{a}$, in which the heavy quark-antiquark pair is replaced by a massless gluon, do not therefore contribute). They are given by

$$
\mathcal{D}_{1}^{a 3(\mathrm{~b})}=\frac{1}{2 p_{a} \cdot p_{3}} \frac{1}{2} \frac{1}{x_{31, a}}\left\langle\mu\left|\mathbf{V}_{1}^{q_{a} q_{3}}\right| \nu\right\rangle \times \frac{1}{N_{\mathrm{c}}^{2}-1} \mathcal{T}_{\mu \nu}\left(\widetilde{p}_{31}, p_{2},-\widetilde{p}_{a}\right),
$$

with $x_{31, a}, \widetilde{p}_{a}$ and $\widetilde{p}_{31}$ again given by Eqs. (D.46) and (D.47) and $\left\langle\mu\left|\mathbf{V}_{1}^{q_{a} q_{3}}\right| \nu\right\rangle$ given in Eq. (5.83). The dipole contribution $\mathcal{D}_{2}^{a 3}$ can be obtained from $\mathcal{D}_{1}^{a 3}$ by the replacement $p_{1} \leftrightarrow p_{2}$.

Next we need to evaluate the insertion operator $\boldsymbol{I}_{2+a}\left(\epsilon, \mu^{2},\left\{p_{i}, m_{i}\right\}, p_{a}\right)$, which cancels all the singularities of the one-loop cross section, and the finite operators related to the factorization of initial-state singularities, $\boldsymbol{P}_{2}^{a, a^{\prime}}\left(x ; \mu_{F}^{2} ;\left\{p_{i}\right\}, x p_{a}\right)$ and $\boldsymbol{K}_{2}^{a, a^{\prime}}\left(x ;\left\{p_{i}, m_{i}\right\}, x p_{a}\right)$.

The expression for $\boldsymbol{I}_{2+a}(\epsilon)$ is identical to that for $\boldsymbol{I}_{3}(\epsilon)$, Eq. (D.42), except that $\left\{m_{F}\right\}$ is replaced by the empty set, \{\} , and $\kappa$ is replaced by $2 / 3$. Note that our uniform notation (see the discussion after Eq. (C.27) of Ref. [11]) means that all dot products, $s_{j a}$, remain positive and that $s$ should be replaced by $Q^{2}=-\left(p_{a}-p_{1}-p_{2}\right)^{2}$.

The operators $\boldsymbol{P}_{2}^{a, a^{\prime}}$ are independent of the presence of massive quarks in the final state and are given by

$$
\begin{gathered}
\sum_{a^{\prime}} 2, a^{\prime}\left\langle 1,2 ; x p_{a}\left|\boldsymbol{P}_{2}^{q, a^{\prime}}\left(x ; \mu_{F}^{2} ; p_{1}, p_{2}, x p_{a}\right)\right| 1,2 ; x p_{a}\right\rangle_{2, a^{\prime}}= \\
-\frac{\alpha_{\mathrm{s}}}{2 \pi} P^{q g}(x) \ln \frac{\mu_{F}^{2}}{x \sqrt{s_{1 a} s_{2 a}}} \times \frac{1}{N_{\mathrm{c}}^{2}-1}\left|\mathcal{M}_{3}\left(p_{1}, p_{2},-x p_{a}\right)\right|^{2}, \\
\sum_{a^{\prime}} 2, a^{\prime}\left\langle 1,2 ; x p_{a}\left|\boldsymbol{P}_{2}^{g, a^{\prime}}\left(x ; \mu_{F}^{2} ; p_{1}, p_{2}, x p_{a}\right)\right| 1,2 ; x p_{a}\right\rangle_{2, a^{\prime}}= \\
-\frac{\alpha_{\mathrm{s}}}{2 \pi} P^{g g}(x) \ln \frac{\mu_{F}^{2}}{x \sqrt{s_{1 a} s_{2 a}}} \times \frac{1}{N_{\mathrm{c}}^{2}-1}\left|\mathcal{M}_{3}\left(p_{1}, p_{2},-x p_{a}\right)\right|^{2},
\end{gathered}
$$

for incoming quarks, $a=q$, and gluons, $a=g$, respectively.

The operators $\boldsymbol{K}_{2}^{a, a^{\prime}}$ do depend on the quark mass and are factorization-scheme, but not -scale, dependent. They are given by

$$
\begin{aligned}
\sum_{a^{\prime}} 2, a^{\prime} & \left\langle 1,2 ; x p_{a}\left|\boldsymbol{K}_{2}^{q, a^{\prime}}\left(x, p_{1}, m_{Q}, p_{2}, m_{Q}, x p_{a}\right)\right| 1,2 ; x p_{a}\right\rangle_{2, a^{\prime}}= \\
\frac{\alpha_{\mathrm{s}}}{2 \pi} & {\left[\bar{K}^{q g}(x)-K_{\mathrm{F.S.}}^{q g}(x)+\frac{1}{2} C_{\mathrm{F}}\left(\frac{C_{\mathrm{A}}}{C_{\mathrm{F}}} \mathcal{K}_{q}^{q, g}\left(x ; s_{1 a}, m_{Q}\right)+\frac{C_{\mathrm{A}}}{C_{\mathrm{F}}} \mathcal{K}_{q}^{q, g}\left(x ; s_{2 a}, m_{Q}\right)\right)\right.} \\
& \left.+\frac{1}{2} P_{\mathrm{reg}}^{q g}(x)\left(\ln \frac{(1-x) s_{1 a}}{(1-x) s_{1 a}+m_{Q}^{2}}+\ln \frac{(1-x) s_{2 a}}{(1-x) s_{2 a}+m_{Q}^{2}}\right)\right]
\end{aligned}
$$




$$
\begin{aligned}
\times & \frac{1}{N_{\mathrm{c}}^{2}-1}\left|\mathcal{M}_{3}\left(p_{1}, p_{2},-x p_{a}\right)\right|^{2} \\
\sum_{a^{\prime}} 2, a^{\prime} & \left\langle 1,2 ; x p_{a}\left|\boldsymbol{K}_{2}^{g, a^{\prime}}\left(x, p_{1}, m_{Q}, p_{2}, m_{Q}, x p_{a}\right)\right| 1,2 ; x p_{a}\right\rangle_{2, a^{\prime}}= \\
\frac{\alpha_{\mathrm{S}}}{2 \pi}\left[\bar{K}^{g g}(x)-K_{\mathrm{FS}}^{g g}(x)+\frac{1}{2} C_{\mathrm{A}}\left(\mathcal{K}_{q}^{g, g}\left(x ; s_{1 a}, m_{Q}\right)+\mathcal{K}_{q}^{g, g}\left(x ; s_{2 a}, m_{Q}\right)\right)\right. & (\mathrm{D} .52) \\
& +\frac{1}{2} \gamma_{g} \delta(1-x)\left(\ln \frac{s_{1 a}-2 m_{Q} \sqrt{s_{1 a}+m_{Q}^{2}}+2 m_{Q}^{2}}{s_{1 a}}+\ln \frac{s_{2 a}-2 m_{Q} \sqrt{s_{2 a}+m_{Q}^{2}}+2 m_{Q}^{2}}{s_{2 a}}\right) \\
& \left.+\frac{1}{2} P_{\mathrm{reg}}^{g g}(x)\left(\ln \frac{(1-x) s_{1 a}}{(1-x) s_{1 a}+m_{Q}^{2}}+\ln \frac{(1-x) s_{2 a}}{(1-x) s_{2 a}+m_{Q}^{2}}\right)\right] \\
\times & \frac{1}{N_{\mathrm{c}}^{2}-1}\left|\mathcal{M}_{3}\left(p_{1}, p_{2},-x p_{a}\right)\right|^{2},
\end{aligned}
$$

for incoming quarks and gluons respectively. The functions $\bar{K}^{a a^{\prime}}, \mathcal{K}_{q}^{q, g}, \mathcal{K}_{q}^{g, g}$ and $P_{\text {reg }}^{a b}$ are defined in Eqs. (6.56), (6.59), (6.60) and (5.89) respectively, while $K_{\mathrm{F.S}}^{a a^{\prime}}$ define the factorization scheme $\left(K_{\mathrm{F} . \mathrm{S}}^{a a^{\prime}}(x)=0\right.$ in the $\overline{\mathrm{MS}}$ scheme) as discussed in Ref. [11].

The ingredients given above are sufficient to construct a complete NLO calculation for any observable that requires the presence of heavy quarks in the final state. The dipole contributions are subtracted from the real matrix elements as in Eq. (6.6) to give a finite 3parton integral. The operator $\boldsymbol{I}_{2+a}(\epsilon)$ is inserted into Eq. (6.8), cancelling the singularities in the one-loop matrix element to give a finite 2-parton integral. Finally, the operators $\boldsymbol{P}_{2}^{a, a^{\prime}}\left(x ; \mu_{F}^{2}\right)$ and $\boldsymbol{K}_{2}^{a, a^{\prime}}(x)$ are inserted into Eq. (6.9) to give the finite remainder from initial-state factorization.

However, for a complete description of all final states in deep inelastic scattering, one must also consider heavy-quark corrections to light-quark processes. Since we do not consider incoming massive partons, the only such correction comes from the 'QCD Compton process', $e+q \rightarrow e+q+g$, followed by the gluon decay $g \rightarrow Q+\bar{Q}$. Rather than giving all the formulae relevant for a complete NLO calculation of the QCD Compton process, we assume that such a calculation already exists with $N_{f}$ massless quark flavours and give the additional terms that must be added to it owing to the presence of the $N_{F}$ massive quark flavours.

Firstly, we have to evaluate the two dipole corrections we neglected in process (b) earlier, $\mathcal{D}_{12,3}$ and $\mathcal{D}_{12}^{a}$, in which the massive quark-antiquark pair is replaced by a massless gluon. The first is constructed in exactly the same way as in the $e^{+} e^{-}$processes discussed earlier. The second, with a final-state emitter and initial-state spectator, is given by (recall that the momenta are labelled $\left.e+q\left(p_{a}\right) \rightarrow e+Q\left(p_{1}\right)+\bar{Q}\left(p_{2}\right)+q\left(p_{3}\right)\right)$,

$$
\mathcal{D}_{12}^{a}=\frac{1}{2 p_{1} \cdot p_{2}+2 m_{Q}^{2}} \frac{1}{2} \frac{1}{x_{12, a}}\left\langle\mu\left|\mathbf{V}_{Q_{1} \bar{Q}_{2}}^{a}\right| \nu\right\rangle \times \frac{1}{N_{\mathrm{c}}} \mathcal{T}_{\mu \nu}\left(p_{3},-\widetilde{p}_{a}, \widetilde{p}_{12}\right),
$$

with

$$
\begin{gathered}
x_{12, a}=\frac{p_{a} \cdot p_{1}+p_{a} \cdot p_{2}-p_{1} \cdot p_{2}-m_{Q}^{2}}{p_{a} \cdot p_{1}+p_{a} \cdot p_{2}} \\
\widetilde{p}_{a}^{\mu}=x_{12, a} p_{a}^{\mu}, \quad \widetilde{p}_{12}^{\mu}=p_{1}^{\mu}+p_{2}^{\mu}-\left(1-x_{12, a}\right) p_{a}^{\mu},
\end{gathered}
$$


and $\left\langle\mu\left|\mathbf{V}_{Q_{1} \bar{Q}_{2}}^{a}\right| \nu\right\rangle$ in Eq. (5.51). The matrix element for the Born process, $e+q\left(p_{a}\right) \rightarrow$ $e+q\left(p_{1}\right)+g\left(p_{2}\right)$, not summed over gluon spin, is given by $\frac{1}{N_{\mathrm{c}}} \mathcal{T}_{\mu \nu}\left(p_{1},-p_{a}, p_{2}\right)$.

Next we have to evaluate the additional contribution to the operator $\boldsymbol{I}(\epsilon)$ from the $N_{F}$ flavours of massive quark,

$$
\begin{aligned}
{ }_{2, a}\left\langle 1,2 ; a\left|\delta \boldsymbol{I}_{2+a}(\epsilon)\right| 1,2 ; a\right\rangle_{2, a} \\
\equiv{ }_{2, a}\left\langle 1,2 ; a\left|\boldsymbol{I}_{2+a}\left(\epsilon,\left\{m_{F}\right\}\right)\right| 1,2 ; a\right\rangle_{2, a}-{ }_{2, a}\left\langle 1,2 ; a\left|\boldsymbol{I}_{2+a}(\epsilon,\{\})\right| 1,2 ; a\right\rangle_{2, a} \\
=\frac{\alpha_{\mathrm{s}}}{2 \pi} \times \frac{2}{3} T_{\mathrm{R}}\left\{\sum_{F=1}^{N_{F}^{21}}\left[\ln \frac{1+\rho_{F, 21}}{2}-\frac{\rho_{F, 21}}{3}\left(3+\rho_{F, 21}^{2}\right)-\frac{1}{2} \ln \frac{m_{F}^{2}}{s_{21}}\right]\right. \\
\left.\quad+\sum_{F=1}^{N_{F}^{2 a}}\left[\ln \frac{1+\rho_{F, 2 a}}{2}-\frac{\rho_{F, 2 a}}{3}\left(3+\rho_{F, 2 a}^{2}\right)-\frac{1}{2} \ln \frac{m_{F}^{2}}{s_{2 a}}\right]\right\} \times \frac{1}{N_{\mathrm{c}}}\left|\mathcal{M}_{3}\left(p_{1},-p_{a}, p_{2}\right)\right|^{2}
\end{aligned}
$$

with $\rho_{F, j k}=\sqrt{1-4 m_{F}^{2} / s_{j k}}$ and $N_{F}^{j k}$ defined to be the number of flavours for which $s_{j k}>$ $4 m_{F}^{2}$. Note that this contribution is finite, so we have set $\epsilon \rightarrow 0$. It does however diverge as $m_{F} \rightarrow 0$, which is cancelled by a corresponding divergence from the virtual matrix element, yielding a two-parton integral that has a smooth small-mass limit.

The operator $\boldsymbol{P}(x)$ is unaffected by the quark mass. Finally, therefore, we just have to evaluate the extra contribution to the operator $\boldsymbol{K}(x)$ from the $N_{F}$ flavours of massive quark,

$$
\begin{aligned}
& \sum_{a^{\prime}} 2, a^{\prime}\left\langle 1,2 ; x p_{a}\left|\delta \boldsymbol{K}^{a, a^{\prime}}(x)\right| 1,2 ; x p_{a}\right\rangle_{2, a^{\prime}} \\
& \equiv \sum_{a^{\prime}} 2, a^{\prime}\left\langle 1,2 ; x p_{a}\left|\boldsymbol{K}^{a, a^{\prime}}\left(x,\left\{m_{F}\right\}\right)\right| 1,2 ; x p_{a}\right\rangle_{2, a^{\prime}}-\sum_{a^{\prime}} 2, a^{\prime}\left\langle 1,2 ; x p_{a}\left|\boldsymbol{K}^{a, a^{\prime}}(x,\{\})\right| 1,2 ; x p_{a}\right\rangle_{2, a^{\prime}} \\
& =\frac{\alpha_{\mathrm{s}}}{2 \pi} \times \frac{2}{3} T_{\mathrm{R}} \sum_{F=1}^{N_{F}^{2 a}}\left\{\left(\delta\left(x_{+}-x\right)-\delta(1-x)\right)\left[\ln \frac{1+\rho_{F, 2 a}}{2}-\frac{\rho_{F, 2 a}}{3}\left(3+\rho_{F, 2 a}^{2}\right)-\frac{1}{2} \ln \frac{m_{F}^{2}}{s_{2 a}}\right]\right. \\
& \left.+\frac{1}{2}\left(\frac{1-x+2 m_{F}^{2} / s_{2 a}}{(1-x)^{2}} \sqrt{1-\frac{4 m_{F}^{2} / s_{2 a}}{1-x}}\right)_{x+}+\delta\left(x_{+}-x\right) \frac{1}{2} \rho_{F, 2 a}^{3}\right\} \times \frac{1}{N_{\mathrm{c}}}\left|\mathcal{M}_{3}\left(p_{1},-x p_{a}, p_{2}\right)\right|^{2} \text {, }
\end{aligned}
$$

for $a=q$, with $x_{+}=1-4 m_{F}^{2} / s_{2 a}=\rho_{F, 2 a}^{2}$. For $a=g, \delta \boldsymbol{K}(x)$ is zero. Note that, as discussed at the ends of Sects. 5.2 and 6.3 and in App. B, $s_{2 a}$ is given by $2 p_{2} \cdot p_{a}$, i.e. it is calculated from $p_{a}$, the momentum of the initial parton, rather than $x p_{a}$, the momentum of the parton entering the Born cross section and from $p_{2}$, the final-state momentum belonging to the boosted phase space.

In the small-mass limit, since $x_{+} \rightarrow 1$, the first line of Eq. (D.60) does not contribute, so the whole contribution remains finite. It is straightforward to check that in this limit, it gives the same results as if the $N_{F}$ extra flavours were massless.

These ingredients, together with the massless results in Ref. [1], are sufficient to provide a complete NLO calculation of all final states in deep inelastic scattering involving either massive or massless partons. However, as discussed in Sect. 2.2, care must be taken in taking the small-mass limit, since we do not include incoming massive partons. Logarithms of $m_{F}^{2}$ 
will therefore remain in the final cross section, preventing a smooth small-mass limit. Since we have explicitly demonstrated that all the insertion operators do have smooth limits, this behaviour is isolated in the three-parton integral, $\int\left(d \sigma^{R}-d \sigma^{A}\right)$. To achieve a smooth small-mass limit, the logarithmic behaviour of the three-parton integral can be evaluated and properly matched with a suitable definition of the heavy-quark parton distribution (see Ref. 42]).

\section{References}

[1] Proceedings of the Workshop on Standard model physics (and more) at the LHC, Eds. G. Altarelli and M.L. Mangano, CERN-2000-04, Geneva 2000.

[2] E. Accomando et al. [ECFA/DESY LC Physics Working Group Collaboration], Phys. Rept. 299 (1998) 1 [hep-ph/9705442;

J. A. Aguilar-Saavedra et al., TESLA Technical Design Report Part III: Physics at an $\mathrm{e}^{+} \mathrm{e}^{-}$Linear Collider, hep-ph/0106315.

[3] P. Nason, S. Dawson and R. K. Ellis, Nucl. Phys. B 303 (1988) 607;

W. Beenakker, H. Kuijf, W. L. van Neerven and J. Smith, Phys. Rev. D 40 (1989) 54.

[4] P. Nason, S. Dawson and R. K. Ellis, Nucl. Phys. B 327 (1989) 49 [Erratum-ibid. B 335 (1989) 260];

M. L. Mangano, P. Nason and G. Ridolfi, Nucl. Phys. B 373 (1992) 295.

[5] W. T. Giele and E. W. Glover, Phys. Rev. D 46 (1992) 1980.

[6] W. T. Giele, E. W. Glover and D. A. Kosower, Nucl. Phys. B 403 (1993) 633 [hep$\mathrm{ph} / 9302225$.

[7] S. Keller and E. Laenen, Phys. Rev. D 59 (1999) 114004 hep-ph/9812415.

[8] B. W. Harris and J. F. Owens, preprint ANL-HEP-PR-00-044 hep-ph/0102128.

[9] S. Frixione, Z. Kunszt and A. Signer, Nucl. Phys. B 467 (1996) 399 hep-ph/9512328;

S. Frixione, Nucl. Phys. B 507 (1997) 295 [hep-ph/9706545].

[10] S. Catani and M. H. Seymour, Phys. Lett. B 378 (1996) 287 hep-ph/9602277.

[11] S. Catani and M. H. Seymour, Nucl. Phys. B 485 (1997) 291 [Erratum-ibid. B 510 (1997) 291] hep-ph/9605323.

[12] Z. Nagy and Z. Trócsányi, Nucl. Phys. B 486 (1997) 189 hep-ph/9610498.

[13] K. Fabricius, I. Schmitt, G. Kramer and G. Schierholz, Z. Phys. C 11 (1981) 315;

G. Kramer and B. Lampe, Fortsch. Phys. 37 (1989) 161.

[14] R. K. Ellis, D. A. Ross and A. E. Terrano, Nucl. Phys. B 178 (1981) 421.

[15] Z. Nagy and Z. Trócsányi, Phys. Rev. Lett. 79 (1997) 3604 hep-ph/9707309, Phys. Rev. D 57 (1998) 5793 hep-ph/9712385, Phys. Rev. D 59 (1999) 014020 [Erratumibid. D 62 (1999) 099902] [hep-ph/9806317]. 
[16] S. Weinzierl and D. A. Kosower, Phys. Rev. D 60 (1999) 054028 hep-ph/9901277.

[17] Z. Nagy and Z. Trócsányi, Phys. Rev. Lett. 87 (2001) 082001 hep-ph/0104315.

[18] Z. Nagy, preprint IPPP/01/48 hep-ph/0110315.

[19] J. M. Campbell and R. K. Ellis, Phys. Rev. D 60 (1999) 113006 hep-ph/9905386.

[20] R. K. Ellis and S. Veseli, Phys. Rev. D 60 (1999) 011501 hep-ph/9810489;

J. M. Campbell and R. K. Ellis, Phys. Rev. D 62 (2000) 114012 [hep-ph/0006304.

[21] W. Beenakker, M. Klasen, M. Krämer, T. Plehn, M. Spira and P. M. Zerwas, Phys. Rev. Lett. 83 (1999) 3780 hep-ph/9906298.

[22] E. Maina, R. Pittau and M. Pizzio, Phys. Lett. B 393 (1997) 445 hep-ph/9609468], Phys. Lett. B 429 (1998) 354 [hep-ph/9710375].

[23] S. Catani and M. H. Seymour, JHEP 9907 (1999) 023 hep-ph/9905424.

[24] M. Krämer, Phys. Rev. D 60 (1999) 111503 hep-ph/9904416].

[25] S. Dittmaier, Nucl. Phys. B 565 (2000) 69 hep-ph/9904440.

[26] M. Roth, PhD thesis, ETH Zürich No. 13363 (1999), hep-ph/0008033.

[27] A. Denner, S. Dittmaier, M. Roth and D. Wackeroth, Nucl. Phys. B 587 (2000) 67 [hep-ph/0006307.

[28] S. Dittmaier and M. Krämer, preprint DESY-01-121, to appear in Phys. Rev. D [hepph/0109062].

[29] S. Dittmaier, M. Krämer, Y. Liao, M. Spira and P. M. Zerwas, Phys. Lett. B 478 (2000) 247 hep-ph/0002035.

[30] L. Phaf and S. Weinzierl, JHEP 0104 (2001) 006 [hep-ph/0102207].

[31] S. Catani, S. Dittmaier and Z. Trócsányi, Phys. Lett. B 500 (2001) 149 hep$\mathrm{ph} / 0011222$.

[32] W. Beenakker, S. Dittmaier, M. Krämer, B. Plümper, M. Spira and P. M. Zerwas, Phys. Rev. Lett. 87 (2001) 201805 hep-ph/0107081.

[33] S. Catani and M. H. Seymour, Acta Phys. Polon. B 28 (1997) 863 [hep-ph/9612236].

[34] G. Rodrigo, A. Santamaria and M. Bilenky, Phys. Rev. Lett. 79 (1997) 193 hepph/9703358, Nucl. Phys. B 554 (1999) 257 [hep-ph/9905276];

W. Bernreuther, A. Brandenburg and P. Uwer, Phys. Rev. Lett. 79 (1997) 189 [hepph/9703305];

P. Nason and C. Oleari, Nucl. Phys. B 521 (1998) 237 hep-ph/9709360.

[35] See, for instance:

A. Bassetto, M. Ciafaloni and G. Marchesini, Phys. Rept. 100 (1983) 201;

Yu. L. Dokshitzer, V. A. Khoze, A. H. Mueller and S. I. Troian, Basics of perturbative QCD (Editions Frontières, Gif-sur-Yvette, 1991). 
[36] G. Altarelli and G. Parisi, Nucl. Phys. B 126 (1977) 298.

[37] Z. Kunszt, A. Signer and Z. Trócsányi, Nucl. Phys. B 420 (1994) 550 hep-ph/9401294].

[38] See, J. C. Collins, D. E. Soper and G. Sterman, in Perturbative Quantum Chromodynamics, Ed. A.H. Mueller (World Scientific, Singapore, 1989), p. 1, and references therein.

[39] F. Bloch and A. Nordsieck, Phys. Rev. 52 (1937) 54.

[40] R. Doria, J. Frenkel and J. C. Taylor, Nucl. Phys. B 168 (1980) 93;

C. Di'Lieto, S. Gendron, I. G. Halliday and C. T. Sachrajda, Nucl. Phys. B 183 (1981) 223;

S. Catani, M. Ciafaloni and G. Marchesini, Nucl. Phys. B 264 (1986) 588;

S. Catani, Z. Phys. C 37 (1988) 357.

[41] B. Mele and P. Nason, Nucl. Phys. B 361 (1991) 626;

M. Cacciari and M. Greco, Nucl. Phys. B 421 (1994) 530 [hep-ph/9311260.

[42] R. Demina et al., hep-ph/0005112.

[43] J. Collins, F. Wilczek and A. Zee, Phys. Rev. D 18 (1978) 242;

W. Bernreuther and W. Wetzel, Nucl. Phys. B 197 (1982) 228 [E ibid. B 513 (1982) 758].

[44] Z. Kunszt, A. Signer and Z. Trócsányi, Nucl. Phys. B 411 (1994) 397 hep-ph/9305239].

[45] S. Catani, M. H. Seymour and Z. Trócsányi, Phys. Rev. D 55 (1997) 6819 hepph/9610553.

[46] V. N. Baier, V. S. Fadin and V. A. Khoze, Nucl. Phys. B 65 (1973) 381.

[47] J. Kodaira and L. Trentadue, Phys. Lett. B 123 (1983) 335;

S. Catani and L. Trentadue, Nucl. Phys. B 327 (1989) 323;

S. Catani, E. D'Emilio and L. Trentadue, Phys. Lett. B 211 (1988) 335;

S. Catani, L. Trentadue, G. Turnock and B. R. Webber, Nucl. Phys. B 407 (1993) 3;

S. Catani, B. R. Webber and G. Marchesini, Nucl. Phys. B 349 (1991) 635.

[48] W. Beenakker, R. Höpker, M. Spira and P. M. Zerwas, Nucl. Phys. B 492 (1997) 51 hep-ph/9610490.

[49] W. Beenakker, M. Krämer, T. Plehn, M. Spira and P. M. Zerwas, Nucl. Phys. B 515 (1998) 3 hep-ph/9710451.

[50] E. L. Berger, M. Klasen and T. M. Tait, Phys. Rev. D 62 (2000) 095014 hepph/0005196.

[51] M. Krämer, T. Plehn, M. Spira and P. M. Zerwas, Phys. Rev. Lett. 79 (1997) 341 hep-ph/9704322].

[52] S. P. Martin and M. T. Vaughn, Phys. Lett. B 318, 331 (1993) hep-ph/9308222.

[53] H. P. Nilles, Phys. Rev. Lett. 45, 319 (1980). 
[54] J. Jersák, E. Laermann and P. M. Zerwas, Phys. Rev. D 25 (1982) 1218 [Erratum-ibid. D 36 (1982) 310].

[55] B. A. Kniehl and J. H. Kühn, Nucl. Phys. B 329 (1990) 547.

[56] T. O. Eynck, E. Laenen, L. Phaf and S. Weinzierl, preprint NIKHEF 2001-013 hep$\mathrm{ph} / 0109246$.

[57] A. Ballestrero, E. Maina and S. Moretti, Nucl. Phys. B 415 (1994) 265 hep$\mathrm{ph} / 9212246$.

[58] M. H. Seymour, Nucl. Phys. B 436 (1995) 163.

D. J. Miller and M. H. Seymour, Phys. Lett. B 435 (1998) 213 hep-ph/9805414].

[59] A. Brandenburg and P. Uwer, Nucl. Phys. B 515 (1998) 279 [hep-ph/9708350].

[60] B. W. Harris and J. Smith, Phys. Rev. D 57 (1998) 2806 [hep-ph/9706334. 